\title{
Studies of Helium Based Gas Mixtures Using a Small Cell Drift Chamber
}

Jaret Heise

SLAC-R-830

Prepared for the Department of Energy

under contract number DE-AC02-76SF00515

Printed in the United States of America. Available from the National Technical Information Service, U.S. Department of Commerce, 5285 Port Royal Road, Springfield, VA 22161. 
This document, and the material and data contained therein, was developed under sponsorship of the United States Government. Neither the United States nor the Department of Energy, nor the Leland Stanford Junior University, nor their employees, nor their respective contractors, subcontractors, or their employees, makes an warranty, express or implied, or assumes any liability of responsibility for accuracy, completeness or usefulness of any information, apparatus, product or process disclosed, or represents that its use will not infringe privately owned rights. Mention of any product, its manufacturer, or suppliers shall not, nor is it intended to, imply approval, disapproval, or fitness of any particular use. A royalty-free, nonexclusive right to use and disseminate same of whatsoever, is expressly reserved to the United States and the University. 


\title{
STUDIES OF HELIUM-BASED GAS MIXTURES USING A SMALL CELL DRIFT CHAMBER
}

\author{
By \\ Jaret Heise \\ B.Sc.(Hon), University of Saskatchewan, 1993 \\ A THESIS SUBMITTED IN PARTIAL FULFILLMENT OF \\ THE REQUIREMENTS FOR THE DEGREE OF \\ MASTER OF SCIENCE \\ in \\ THE FACULTY OF GRADUATE STUDIES \\ DEPARTMENT OF PHYSICS \\ We accept this thesis as conforming \\ to the required standard
}

THE UNIVERSITY OF BRITISH COLUMBIA

March 1996

(c) Jaret Heise, 1996 
In presenting this thesis in partial fulfillment of the requirements for an advanced degree at the University of British Columbia, I agree that the Library shall make it freely available for reference and study. I further agree that permission for extensive copying of this thesis for scholarly purposes may be granted by the head of my department or by his or her representatives. It is understood that copying or publication of this thesis for financial gain shall not be allowed without my written permission.

Department of Physics

The University of British Columbia

6224 Agricultural Road

Vancouver, BC, Canada

V6T $1 Z 1$

Date: 


\section{Abstract}

An international collaboration is currently working on the construction and design of an asymmetric B Factory at the Stanford Linear Accelerator Center that will be ready to collect data in 1999. The main physics motivation for such a facility is to test the description and mechanism of $C P$ violation in the Standard Model of particle physics and provide insight into the question of why more matter than antimatter is observed in the universe today. In particular, this experiment will measure $C P$ violation in the decay of $B$ mesons.

In the early stages of this effort, the Canadian contingent proposed to build the central tracking chamber for the $B_{A} B_{A R}$ detector. Presently, a prototype drift chamber is in operation and studies are being performed to test some of the unique features of drift chamber design dictated by the conditions of the experiment. Using cosmic muons, it is possible to study tracking and pattern recognition in the prototype chamber, and therefore calculate the efficiency and spatial resolution of the prototype chamber cells. These performance features will be used to test whether or not the helium-based gas mixtures proposed for the $B_{A} B_{A R}$ drift chamber are a viable alternative to the more traditional argon-based gases. 


\section{Table of Contents}

$\begin{array}{ll}\text { Abstract } & \text { ii }\end{array}$

List of Tables $\quad$ viii

$\begin{array}{ll}\text { List of Figures } & \text { ix }\end{array}$

Quotes $\quad$ xiii

Acknowledgments $\quad$ xiv

1 Introduction $\quad 1$

1.1 Baryon Asymmetry . . . . . . . . . . . . . . . 2

1.1.1 Baryon Number Violation ............... 2

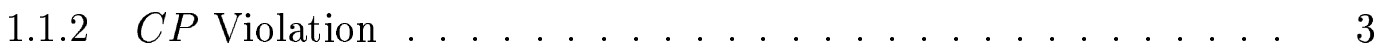

1.1.3 Thermodynamic Equilibrium .............. 4

1.1.4 Summary ........................... 4

$1.2 C P$ Violation at the SLAC B Factory . . . . . . . . . . . . 5

2 CP Violation $\quad 6$

2.1 Introduction . . . . . . . . . . . . . . 6

2.1.1 Violation of Parity . . . . . . . . . . . . 6

2.1.2 Violation of Charge Conjugation . . . . . . . . . 7

2.1.3 CP Symmetry? . . . . . . . . . . . . . . 9 
$2.2 C P$ Violation in the Neutral Kaon System . . . . . . . . . . . 9

$2.3 C P$ Violation in the Standard Model . . . . . . . . . . . . . . 14

$2.4 \quad C P$ Violation in the B Meson System . . . . . . . . . . . . . . . 19

2.4.1 The Physical B Mesons ................ 20

2.4.2 Time Evolution of the Physical State . . . . . . . . . 24

2.4.3 Decays to $C P$ Eigenstates . . . . . . . . . . . 25

2.5 Decay Modes that Measure the Angles of the Unitarity Triangle . . . . 30

2.5.1 The decay $B^{0} \rightarrow \pi^{+} \pi^{-} \ldots \ldots \ldots . \ldots . \ldots 31$

2.5.2 The decay $B^{0} \rightarrow J / \psi K_{S}^{0} \ldots \ldots \ldots . \ldots \ldots$

2.5.3 The decay $B_{s}^{0} \rightarrow \rho K_{S}^{0} \ldots \ldots \ldots \ldots$

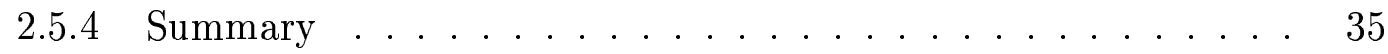

3 The $B_{A} B_{A R}$ Detector $\quad 37$

3.1 Introduction . . . . . . . . . . . . . . . 37

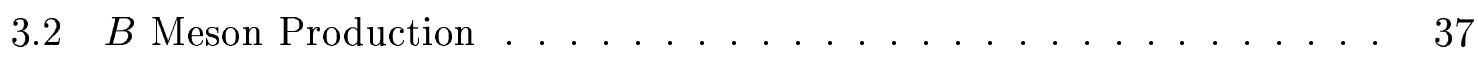

3.2.1 PEP-II Storage Ring . . . . . . . . . . . . . 37

3.2.2 $B^{0}-\overline{B^{0}}$ Orthogonality . . . . . . . . . . . . . 39

3.3 Reconstruction of $B$ Decay Vertices . . . . . . . . . . . . . . 41

3.4 High Luminosity . . . . . . . . . . . . . . . 43

3.5 Multiple Scattering . . . . . . . . . . . . . 45

3.6 Overview of the $B A B A R$ Detector . . . . . . . . . . . . 45

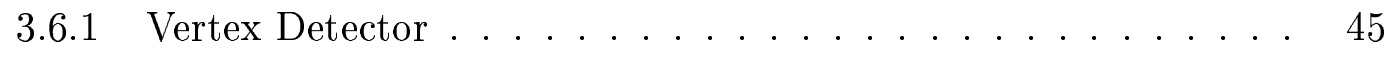

3.6 .2 Drift Chamber . . . . . . . . . . . . . 48

3.6.3 Particle Identification . . . . . . . . . . . . . . 49

3.6.4 Electromagnetic Calorimeter . . . . . . . . . . . 51

3.6.5 Muon and Neutral Hadron Detector . . . . . . . . . . 55 
3.6 .6 Magnet Coil . . . . . . . . . . . . . . . 56

4 Drift Chambers $\quad 59$

4.1 Introduction . . . . . . . . . . . . . . . 59

4.2 Ionization Detectors . . . . . . . . . . . . 59

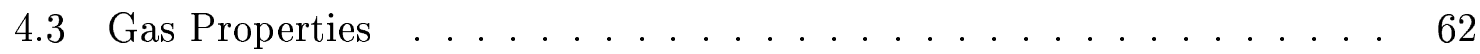

4.3.1 Choice of Gas Type . . . . . . . . . . . . 62

4.3 .2 Drift Velocity ..................... 65

4.3.3 Electron Diffusion . . . . . . . . . . . . . 66

4.4 Wire Configurations ..................... 69

4.5 Behaviour of a Drift Chamber in a Magnetic Field . . . . . . . . . 70

5 Prototype Drift Chamber $\quad 73$

5.1 Introduction . . . . . . . . . . . . . 73

5.2 Prototype Geometry . . . . . . . . . . . . . 73

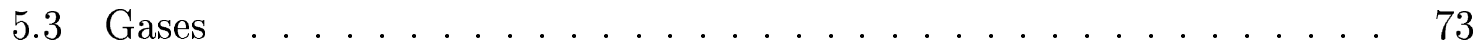

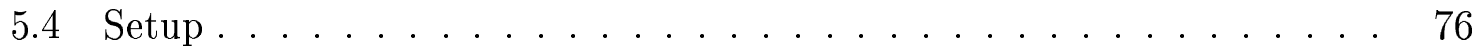

$5.4 .1 \mathrm{TDCs} \ldots \ldots \ldots \ldots \ldots \ldots \ldots$

$5.4 .2 \mathrm{ADCs} \ldots \ldots \ldots \ldots \ldots \ldots \ldots \ldots$

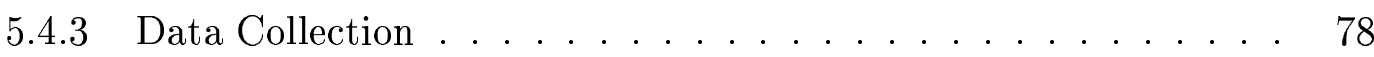

6 Track Fitting $\quad 79$

6.1 Introduction . . . . . . . . . . . . . . . 79

6.2 Data Collection and Storage . . . . . . . . . . 80

6.3 Hardware Preliminaries . . . . . . . . . . . . . 80

6.3 .1 TDC Calibrations . . . . . . . . . . . 80

$6.3 .2 \mathrm{ADCs} \ldots \ldots \ldots \ldots \ldots \ldots \ldots$ 


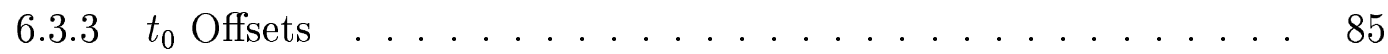

6.4 Segment Finding and Fitting . . . . . . . . . . . . 86

6.4.1 Pattern Recognition . . . . . . . . . . . 86

6.4.2 Left-Right Ambiguities . . . . . . . . . . . . . 88

6.4.3 Segment Fitting . . . . . . . . . . . . 89

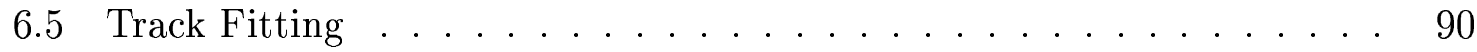

6.6 Track Refitting . . . . . . . . . . . . . . . 94

6.7 Drift Functions . . . . . . . . . . . . . . . 94

$\begin{array}{lll}7 & \text { Efficiency Studies } & 97\end{array}$

7.1 Introduction . . . . . . . . . . . . . . . 97

7.2 Pseudo-efficiency . . . . . . . . . . . . . . . 97

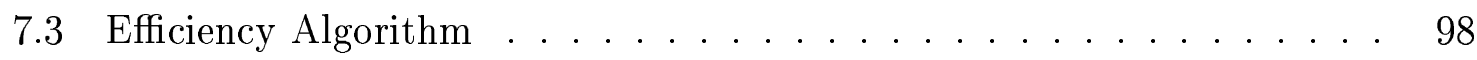

7.4 Analysis Data Reduction . . . . . . . . . . . . 101

7.4.1 Entrance Angle . . . . . . . . . . . . . . 101

7.4 .2 Wires Used ................................ 101

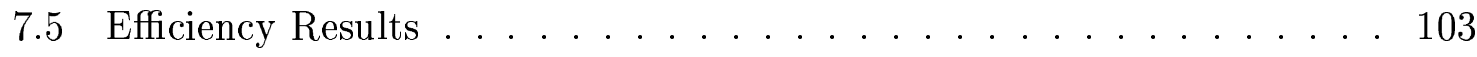

8 Resolution Studies $\quad 112$

8.1 Introduction . . . . . . . . . . . . . . . 112

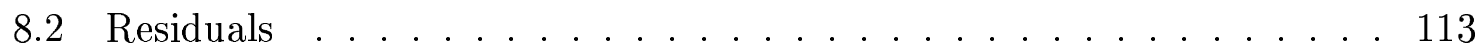

8.3 Fitting Residuals . . . . . . . . . . . . . . . 113

8.4 Resolution Results . . . . . . . . . . . . . . . 119

9 Conclusion $\quad 128$

9.1 Optimum High Voltage . . . . . . . . . . . . 128

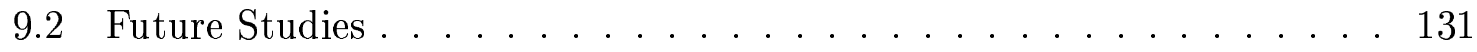




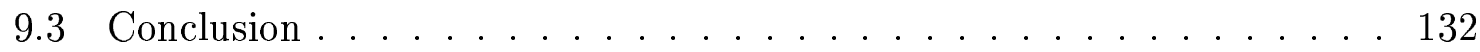

Bibliography

133

Appendix A The $B_{A} B_{A R}$ Collaboration

135 


\section{List of Tables}

2.1 The three generations of fundamental particles. . . . . . . . . . 15

2.2 The relevant decay information and $C P$ physics for each of the proposed

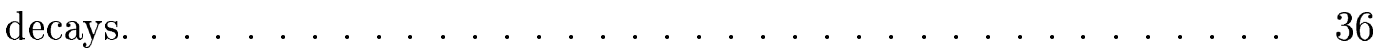

5.3 Properties for various gas mixtures at atmospheric pressure and $20^{\circ} \mathrm{C} . \quad 76$

7.4 Results indicating pseudo-efficiency and average efficiency for the four chamber gases at various operating voltages. . . . . . . . . 106

8.5 The average cell resolution for the four chamber gases at various operating voltages. . . . . . . . . . . . . . . . 122

9.6 Results indicating the optimum operating high voltage for each of the four gas mixtures tested in the prototype chamber. . . . . . . . . . 129 


\section{List of Figures}

2.1 The radioactive $\beta$ decay of ${ }^{60}$ Co before and after a parity transformation. 8

2.2 The action of the charge conjugation symmetry on the neutrino system. 9

2.3 Box diagram for one possible second order weak transition between the kaon and its antiparticle. . . . . . . . . . . . 10

2.4 The standard parameterization of the unitarity triangle as visualized in the complex plane. ...................... 17

2.5 The normalized unitarity triangle as visualized in the complex plane. . 19

2.6 Box diagram for $B$ meson system showing how a $B^{0}$ particle can transform into its antiparticle. . . . . . . . . . . . 20

2.7 Interference between the amplitude for direct decay and the amplitude for indirect decay that proceeds through mixing. . . . . . . . . . 30

2.8 Feynman diagram for the decay $B^{0} \rightarrow \pi^{+} \pi^{-} \ldots \ldots . . . . . . . .31$

2.9 Feynman diagram for the decay $B^{0} \rightarrow J / \psi K_{S}^{0} \ldots \ldots . \ldots . \ldots 33$

2.10 Feynman diagram for the decay $B_{s}^{0} \rightarrow \rho K_{S}^{0}$. . . . . . . . . . 34

3.11 The PEP-II storage ring facility at SLAC. . . . . . . . . . 39

3.12 The orthogonality between the $B^{0}$ and $\overline{B^{0}}$ decay. . . . . . . . . . . 42

3.13 The effects of having (a) equal beam energies, and (b) unequal beam energies when trying to separate decay vertices. . . . . . . . . . 44

3.14 The $B_{A B} B_{A R}$ detector. . . . . . . . . . . . . . . 46

3.15 The silicon vertex tracker $(\mathrm{SVT}) . \ldots \ldots \ldots$. . . . . . . 47 
3.16 Side view of the drift chamber. . . . . . . . . . . . 49

3.17 Side view of the particle identification system. . . . . . . . . 50

3.18 Schematic of a single quartz bar in the DIRC detector illustrating the principle behind Čerenkov counters. . . . . . . . . . . . . 52

3.19 An illustration of the DIRC particle identification system. . . . . . . 53

3.20 The electromagnetic calorimeter. . . . . . . . . . . 54

3.21 The instrumented flux return (IFR) . . . . . . . . . . . 57

3.22 The magnetic field lines of the $1.5 \mathrm{~T}$ magnet. . . . . . . . . . 58

4.23 Schematic of a drift chamber cell. . . . . . . . . . . 60

4.24 Calculated and measured drift velocities as a function of electric field with no magnetic field present. . . . . . . . . . . . 67

4.25 Drift velocity vs. electric field indicating the influence of nitrogen impurities in argon gas. .................... 68

4.26 Diffusion of electrons (a) with no electric field present, and (b) in the presence of an electric field. . . . . . . . . . . . . 69

4.27 Axial and stereo wire configurations. . . . . . . . . . . 71

5.28 The prototype drift chamber wiremap. . . . . . . . . . . 74

5.29 The prototype drift chamber setup. . . . . . . . . . . 77

6.30 TDC channel number vs. time. . . . . . . . . . . . . 81

6.31 Histogram of ADC pulse height per hit using $\mathrm{He}: i-\mathrm{C}_{4} \mathrm{H}_{10}[80: 20]$ gas at an anode voltage of $1850 \mathrm{~V} \ldots \ldots \ldots . \ldots . \ldots 3$

6.32 Measured mean ADC pulse height vs. anode high voltage for the four gases tested in the prototype chamber. . . . . . . . . . . . 84

6.33 Cell pattern of wedge grouping used for segment recognition. . . . . . . 87 
6.34 Valid prototype segment patterns. . . . . . . . . . . . 87

6.35 An illustration of left-right ambiguities. . . . . . . . . . . 88

6.36 An example of how a segment formed with hits from 4 layers can have

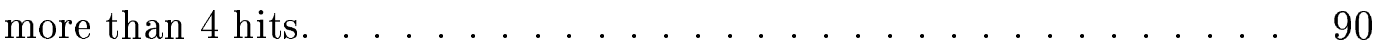

6.37 The definitions of the track parameters $d_{0}$ and $\phi_{0} \ldots \ldots \ldots$

6.38 Drift function for He: $i-\mathrm{C}_{4} \mathrm{H}_{10}[80: 20]$ gas at anode voltage $1850 \mathrm{~V}$. . . 96

7.39 Histogram of the sine of the entrance angle for each hit showing the effect of the cut used in the analysis. . . . . . . . . . . . . . 102

7.40 The prototype wires used in the analysis. . . . . . . . . . . . 103

7.41 Distance of closest approach per hit for $\mathrm{He}: i-\mathrm{C}_{4} \mathrm{H}_{10}$ [80:20] gas at 1850 V.104

7.42 Efficiency vs. anode high voltage for all gases tested in the prototype chamber. . . . . . . . . . . . . . . . . . 105

7.43 Efficiency vs. drift distance using $\mathrm{He}: i-\mathrm{C}_{4} \mathrm{H}_{10}[80: 20]$ gas at various high voltages. . . . . . . . . . . . . . . . . . 108

7.44 Efficiency vs. wire number using $\mathrm{He}: i-\mathrm{C}_{4} \mathrm{H}_{10}[80: 20]$ gas at $1850 \mathrm{~V} \ldots \ldots$

7.45 Efficiency vs. layer number using $\mathrm{He}: i-\mathrm{C}_{4} \mathrm{H}_{10}[80: 20]$ gas at $1850 \mathrm{~V} \ldots \ldots$

7.46 Efficiency vs. layer number for the four gas mixtures averaged over all

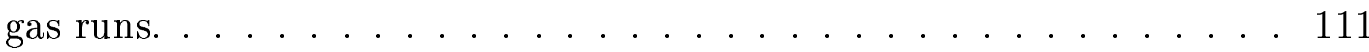

8.47 Residuals for each hit in the data sample before fitting to a Gaussian distribution for He: $i-\mathrm{C}_{4} \mathrm{H}_{10}[80: 20]$ gas at $1850 \mathrm{~V} . \ldots \ldots \ldots$

8.48 Residuals vs. the absolute value of the predicted drift distance obtained through the fitting program using $\mathrm{He}: i-\mathrm{C}_{4} \mathrm{H}_{10}[80: 20]$ gas at $1850 \mathrm{~V} . \quad .115$

8.49 Residuals for each hit using $\mathrm{He}: i-\mathrm{C}_{4} \mathrm{H}_{10}[80: 20]$ gas at $1850 \mathrm{~V}$. . . . 116

8.50 Comparison of fit residual distributions when regions further than $1.5 \sigma$ and $2.0 \sigma$ are excluded using $\mathrm{He}: i-\mathrm{C}_{4} \mathrm{H}_{10}[80: 20]$ gas at $1850 \mathrm{~V} . \ldots$. 
8.51 Comparison of the same residual values fit to 1 or 2 Gaussian distributions using He: $i-\mathrm{C}_{4} \mathrm{H}_{10}[80: 20]$ gas at $1850 \mathrm{~V} \ldots \ldots \ldots$

8.52 Comparison of typical residual values for the four gases tested in the prototype fit using a single Gaussian distribution where regions further than $1.5 \sigma$ on either side of the mean are excluded. . . . . . . . . . 120

8.53 Average resolution vs. anode high voltage for all gases used in the pro-

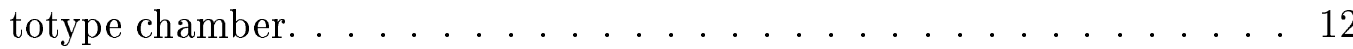

8.54 Resolution vs. drift distance for all gases tested in the prototype at various anode high voltages. . . . . . . . . . . . . . . 124

8.55 Resolution vs. drift distance for each axial layer in the prototype chamber using $\mathrm{He}: i-\mathrm{C}_{4} \mathrm{H}_{10}[80: 20]$ gas at $1850 \mathrm{~V} \ldots \ldots \ldots \ldots$

8.56 Less biased resolution vs. drift distance averaged over all layers in the prototype chamber using $\mathrm{He}: i-\mathrm{C}_{4} \mathrm{H}_{10}[80: 20]$ gas at $1850 \mathrm{~V}$. . . . . . 127

9.57 Efficiency vs. drift distance at the optimum operating high voltage for each gas. . . . . . . . . . . . . . . . . 130

9.58 Resolution vs. drift distance at the optimum operating high voltage for

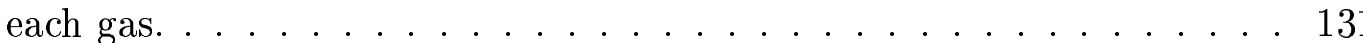




\title{
Quotes
}

In the beginning, the universe was created.

This has made a lot of people very angry and been widely regarded as a bad move.

\author{
-Douglas Adams \\ The Restaurant \\ at the End of the Universe
}

Thou com'st in such a questionable shape

that I will speak to thee...

\author{
-William Shakespeare \\ Hamlet act I, scene 4
}

I believe there are

15747724136275002577605653961181555468044

717914527116709366231425076185631031296

protons in the universe...

-Arthur Eddington
Philosophy of Physical Science

... and slightly fewer antiprotons, perhaps as many as 1 or 2 . 


\section{Acknowledgments}

Of the many people to whom I owe a great debt of thanks, none deserves more gratitude than my supervisor Dr. Janis McKenna. Her energy and enthusiasm are legendary, and her patience for correcting grammar and other typing mistakes is nearly infinite. I suppose if she hadn't been prepared to last for the duration and see me through to the end of my degree, she could have abandoned me in Berkeley or some such place...

Thanks also go to Chris Waltham and Chris Hearty for providing comments and corrections.

My office mates (imaginary or otherwise) at UBC deserve a lot of credit for their constant enthusiasm and understanding. They include Cherie Goodenough, Sarah Dunsiger, Jeff Lange, Trevor Stocki, Gertjan Hofman, Mohammad Kermani and Sheila McFarland. Without these people, I can only assume that I would have been done months sooner. In addition, special thanks go to Cherie for not finishing her thesis first and making me look bad.

For the inspiration of youth, I have Nicola Jones, Ari Benbasat, Peter Michaux and Wesley Wong to thank. One way or the other, Sarah also fits into this category.

Working at SLAC has been a very enriching experience, and as a result I have many people there to thank as well, most notably Steve Schaffner, Mark McDougald, and Dale Pitman. Since I got a shirt out of it, I should probably thank Mike Woods and the rest of the Spinors softball gang, too.

Closer to home, I have my Vancouver relatives: Don, Carol, Jonathan and Matthew to thank for their gracious hospitality, as well as my land people, Ingrid and Terry.

My parents have always been supportive of all my endeavours, and for having 
that environment I consider myself very fortunate. Once they read this thesis, I'm sure they will have a much greater appreciation of the practical benefits that particle physics offers.

Last, but certainly not least, I would like to thank Charles the Cat for helping me with the tricky points of $C P$ violation. Any remaining deficiencies were most likely lost in translation and are entirely my fault. 


\section{Chapter 1}

\section{Introduction}

Imagine the Big Bang that brought the universe into existence approximately 12 billion years ago. The early universe was characterized by a state of extremely high density and high temperature, and as a result of these conditions, two types of particles were present: matter particles and antimatter particles. Without any further knowledge, it would be natural to assume that the universe began in a symmetric state with equal amounts of each type of particle present. If this were not the case, then some explanation would be necessary to account for the difference. It so happens that when matter and antimatter combine, they annihilate in a burst of radiation. Envisioning the universe at this early stage, it therefore necessary to require a predominance of matter particles over antimatter particles. To assume otherwise would mean denying the fact of our very existence, because if the ratio of matter to antimatter was exactly unity, then all of the matter in the universe would have been converted to radiation through annihilation processes billions of years ago, leaving only radiation today and for all of time. Stable matter would not exist to clump together to form planets, stars or galaxies, only photons would inhabit the volume of the universe. The question then becomes: why does anything exist? The obvious answer is that there was slightly more matter than antimatter at some point after the Big Bang, giving rise to the matter dominanted universe we see today, but why?

One possible explanation that was proposed in the past for the matter/antimatter asymmetry suggested that some regions of the universe may contain matter while 
other regions contain antimatter, so that on average there is an equal amount of each type. However, this is not consistent with what is observed. Evidence of antimatter would come in the form of a characteristic photon signal produced when matter and antimatter annihilate. One way to search for antimatter locally is to study the solar wind within our solar system, which consists of streams of charged matter particles that originate from the Sun. If ever this wind encounters a pocket of antimatter, then a photon signal would be emitted, clearly indicating this presence. To date, there has been no indication of antimatter in our solar system or elsewhere, and the argument of distinct matter and antimatter regions applied to larger and larger scales in the universe such as galaxies and clusters of galaxies becomes increasingly difficult to accommodate.

\section{$1.1 \quad$ Baryon Asymmetry}

To explain the matter/antimatter asymmetry of the universe, A.D. Sakharov [1] proposed that three criteria must be satisfied: (i) non-conservation of baryons, $(i i) C P$ violation, and ( $i$ ii) the evolution of the universe from a state of thermodynamic equilibrium to a state of non-equilibrium.

\subsubsection{Baryon Number Violation}

The imbalance in the amount of matter and antimatter can be examined as the imbalance in the amount of baryons and antibaryons, since baryons (neutrons and protons) are the heaviest of the basic constituents of matter. One possible explanation for the asymmetry is that baryons in the early universe were somehow able to decay into particles that were not baryons, or vice versa, that baryons were produced from non-baryons. The final products in baryon decay might have been much lighter, non-baryonic particles such as a lepton (an electron, for instance) and a meson (a pion, for instance) as 
in the process:

$$
p \rightarrow e^{+} \pi^{0}
$$

So far, no experimental evidence has been found to suggest that the proton undergoes a decay of any sort. Experimentally, a lower bound of approximately $5 \times 10^{32}$ years has been placed on the mean lifetime of the proton [2]. Recall that the age of the universe is roughly $10^{10}$ years.

\subsection{2 $C P$ Violation}

Left by itself, the statement of non-conservation of baryons in the first Sakharov condition would produce an equal number of baryons and antibaryons that would eventually annihilate, leaving behind neither matter nor antimatter. A second condition is therefore required in which baryon interactions must violate $C P$ conservation. The exact description of $C P$ violation will be discussed in more detail in Chapter 2, but essentially the $C P$ symmetry relates a particle to its antiparticle. Studying reactions that are the $C P$ conjugates of one another helps in the understanding of the matter/antimatter asymmetry. If a process is able to make an absolute distinction between matter and antimatter, this could lead to differences in how much of each type is produced. For instance, suppose a particle could decay through two channels, one producing matter and the second producing antimatter:

$$
\text { (i) particle } \rightarrow \text { matter }, \quad \text { (ii) particle } \rightarrow \text { antimatter } \text {. }
$$

If one of these decay modes was favoured over the other or happened faster, then this would lead to a difference in the amount of matter and antimatter produced. 


\subsubsection{Thermodynamic Equilibrium}

The final ingredient necessary to create a lasting baryon asymmetry is that the universe must evolve from a state of thermodynamic equilibrium into a state of non-equilibrium. This way, $C P$ processes that favour the production of matter over antimatter can be strung together in time to yield the observed matter dominance. Without this last condition, any matter/antimatter asymmetry would be erased as the state returns to thermodynamic equilibrium. Consider the hot Big Bang in which the temperature of the early universe is very high, high enough in fact that the processes that turn particles into radiation are balanced by reactions that turn radiation into particles. To have matter today however, the universe would have had to cool in order to break the state of equilibrium between matter (and antimatter) and radiation, and in doing so favour matter production over radiation production. It turns out that reactions producing matter prevail because they lead to lower energy states.

Furthermore, a state that is in thermodynamic equilibrium has maximum entropy, or maximum randomness. By the second law of thermodynamics, if a system undergoes change, it will evolve in such as way that its entropy will increase or, at best, remain the same. A difference in the number of particles and antiparticles however decreases the randomness of the system, therefore the system cannot be in a state of equilibrium.

\subsubsection{Summary}

The current scenario for the evolution of the universe is that initially, after the Big Bang, baryons and antibaryons existed in equal proportions. At some later point, processes that violate $C P$ and baryon number occur which yield an excess of baryons over antibaryons, and are preserved by having the universe be temporarily out of thermodynamic equlibrium. A short time after these events take place, the baryon 
number violating reactions are no longer active, and the baryon excess in the universe is "frozen in". Baryons and antibaryons subsequently annihilate:

$$
\text { baryon }+\overline{\text { baryon }} \rightarrow \gamma,
$$

leaving only baryons to populate the universe because all of the antibaryons have since been converted to photons. One characteristic of the matter/antimatter asymmetry that is observed today is the ratio of the number of baryons, $n_{\text {baryon }}$, to the number of photons, $n_{\text {photon }}[3]$ :

$$
\frac{n_{\text {baryon }}-\bar{n}_{\text {baryon }}}{n_{\text {baryon }}+\bar{n}_{\text {baryon }}} \rightarrow \frac{n_{\text {baryon }}}{n_{\gamma}} \simeq 10^{-9} .
$$

\section{2 $C P$ Violation at the SLAC B Factory}

It is the second of the Sakharov criteria, $C P$ violation, that the Stanford Linear Accelerator Center (SLAC) B Factory is being built to study. Using $B^{0}$ mesons in which the manifestation of $C P$ violation is expected to be the most apparent, an international team of scientists is hoping to understand at last one very important aspect of why we exist.

In order to study $C P$ violation, a detector is necessary to measure the key signatures that indicate $C P$ symmetry has been broken. Such a detector will have a number of sub-detector components that will concentrate on different aspects of the experiment, including a drift chamber that will mainly be concerned with measuring the momentum of charged particles. The focus of this thesis will be to outline a series of studies performed using a prototype drift chamber, the results of which will be used in determining certain design features of the full scale detector. 


\section{Chapter 2}

\section{CP Violation}

\subsection{Introduction}

As mentioned in Chapter 1, $C P$ violation might explain why there is more matter in the universe today than antimatter. But what exactly is $C P$ violation?

Physicists often study the symmetries of various systems in order to understand them. $C P$ is a combination of two such symmetries in nature: $P$ being parity or space inversion and $C$ being charge conjugation or particle-antiparticle interchange.

\subsubsection{Violation of Parity}

Prior to 1956, parity was assumed to be a symmetry to which nature adhered, meaning that the mirror image of any process represents another possible process that could be observed in nature. However in 1956, T.D. Lee and C.N. Yang [4] noticed that parity symmetry had been shown to hold for all forces in nature except the force called the weak force which governs radioactive decays, and they proposed to test this assumption. The first and most famous experiment to test parity conservation in weak decays was performed by C.S. Wu [5] one year later using $\beta$ decay of cobalt- 60 . The goal of the experiment was to observe a spatial asymmetry in the emission of electrons from the radioactive decay which could lead to a distinction between the decay process and its mirror image. 
The process used in Wu's experiment was the radioactive decay of cobalt into nickel:

$$
{ }^{60} \mathrm{Co} \rightarrow{ }^{60} \mathrm{Ni}+e^{-}+\bar{\nu}_{e} .
$$

In order to establish a reference direction in space, a magnetic field was applied to the reaction which aligned the spin of the cobalt atom ${ }^{1}$ and therefore the spin of the resulting nickel atom in the same direction as the field. By measuring the emission of electrons parallel or antiparallel to the orientation of the nuclear spin (the direction of the magnetic field), any asymmetry due to a parity transformation can be detected. $\mathrm{Wu}$ and her colleagues observed that in the lab decay, electrons were emitted along the direction of the nuclear spin direction. If parity was to be invariant, then the mirror image process would also have to emit electrons in this same orientation, parallel to the direction of the spin. The nucleus in the mirror image has the direction of its spin flipped because it is now rotating in the opposite direction, yet the electrons in this mirror image reaction are emitted in the direction opposite to the spin as shown in Figure 2.1.

This was clear evidence of a process whose mirror image does not occur in nature. If parity had no effect on this decay, then electrons in the mirror image scenario should have been emitted in the "downward" direction, in the same direction as the spin. However, this was not the case, and the same results were found for all systems governed by the weak force. The weak interaction maximally violates parity; that is, the parity symmetry is broken in every weak interaction.

\subsubsection{Violation of Charge Conjugation}

Another symmetry that is valid for all forces except the weak force is the charge conjugation operation, $C$, which converts each particle into its antiparticle without

\footnotetext{
${ }^{1}$ The most demanding aspect of this experiment was the development of the cryostatic technology needed in order to cool the particles to align the direction of the particle spins.
} 

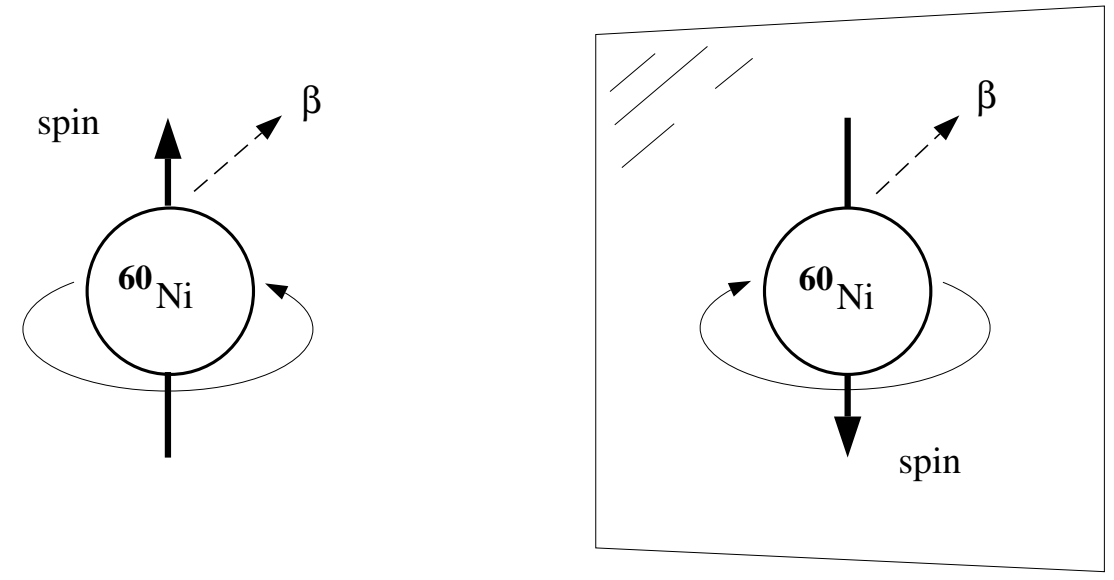

$l a b$

mirror image

Figure 2.1: Diagram showing the radioactive $\beta$ decay of ${ }^{60}$ Co before and after a parity transformation. In the lab, most of the electrons are emitted in the direction of the nuclear spin orientation. Whereas in the mirror image, where the parity operation changes the direction of the spin of the nickel atom, most of the electrons are emitted opposite to the spin direction. This implies directly that parity symmetry is broken.

changing the spin or momentum of the particle. The neutrino, which is a product of weak interactions, is an obvious exception to this symmetry. All neutrinos are lefthanded and all antineutrinos are right-handed. This means that the spin of the neutrino is always aligned antiparallel to its momentum, while the spin of the antineutrino is always parallel to its momentum. Applying the charge conjugation symmetry to a lefthanded neutrino as illustrated in Figure 2.2 gives a left-handed antineutrino in return which does not exist in nature. Therefore, charge conjugation applied to any process involving a neutrino will not result in a physically observed process, which means that the charge conjugation is not a symmetry of weak interactions. 

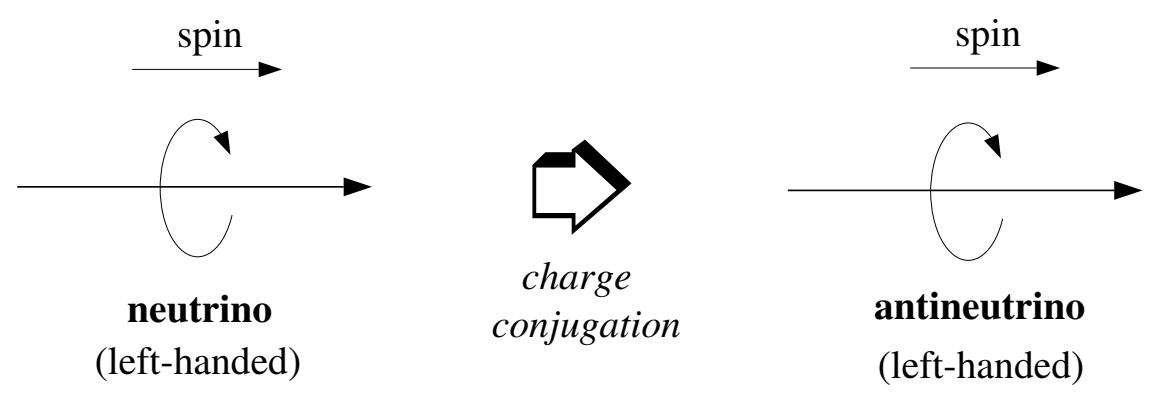

Figure 2.2: Diagram illustrating the action of the charge conjugation symmetry on the neutrino system. The operation of charge conjugation changes a left-handed neutrino, which has been observed in nature, into a left-handed antineutrino, which has not been observed in nature. Therefore, charge conjugation is violated in weak interactions.

\subsubsection{CP Symmetry?}

Even though each of the symmetries $C$ and $P$ is not conserved in weak interactions, physicists thought that perhaps these two symmetry violations would occur coupled together so that the combined $C P$ symmetry would be preserved. For example, in the ${ }^{60}$ Co experiment that was used to demonstrate the violation of parity in weak decays, applying an additional charge conjugation transformation to the mirror image leads to a process in which the particles are emitted along the direction of the spin orientation, exactly as in the original reaction. It would seem then that the combination of $C$ and $P$ together is the symmetry that is upheld in weak interactions, rather than $C$ and $P$ separately, but in $1964 C P$ violation was observed experimentally in the neutral kaon system [6].

\section{2 $C P$ Violation in the Neutral Kaon System}

Unlike a neutral meson such as the pion which is its own antiparticle, the neutral kaon, $K^{0}$, has an associated antiparticle, $\overline{K^{0}}$, which is a distinct state. However a $K^{0}$ particle, 
which is composed of a down type quark and an antistrange type quark, can change into its antiparticle, the $\overline{K^{0}}$, which is made of an antidown quark and and a strange quark. The process that allows this mixing to happen is called a second order weak transition because two mediating $W$ bosons are exchanged as illustrated in Figure 2.3. This mixing property in stable particles is unique to only two other systems, namely the $D$ mesons $^{2}$ and the $B$ mesons.

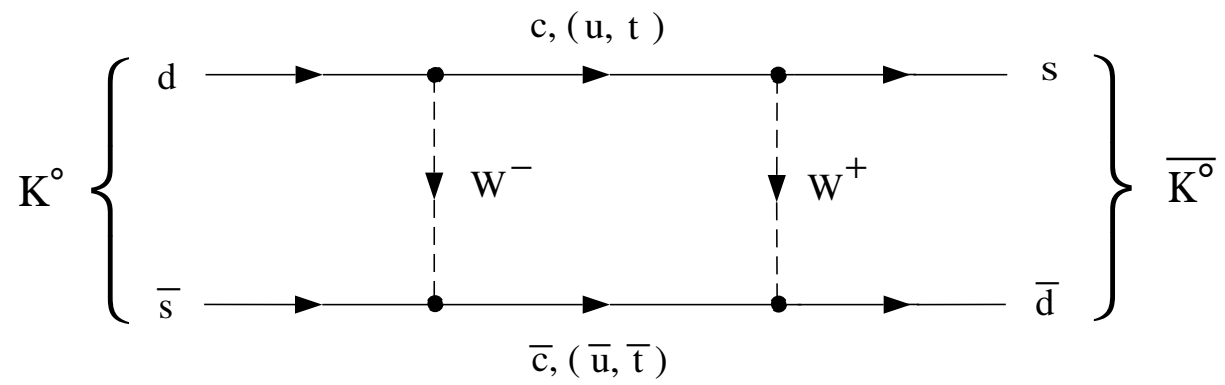

Figure 2.3: Box diagram for one possible second order weak transition between the kaon and its antiparticle.

Now, consider how the symmetries of parity, $P$, and charge conjugation, $C$, act on the kaon states. All mesons including the neutral kaon have negative intrinsic parity, so that when the parity operation is applied to a kaon state, the same kaon state is returned, but with a negative parity eigenvalue:

$$
P\left|K^{0}\right\rangle=-\left|K^{0}\right\rangle, \quad P\left|\overline{K^{0}}\right\rangle=-\left|\overline{K^{0}}\right\rangle .
$$

Charge conjugation is straight forward; as discussed in Section 2.1, the operator $C$ takes a system from the particle state to the corresponding antiparticle state:

$$
C\left|K^{0}\right\rangle=\left|\overline{K^{0}}\right\rangle, \quad C\left|\overline{K^{0}}\right\rangle=\left|K^{0}\right\rangle
$$

\footnotetext{
${ }^{2}$ Although not yet observed, $C P$ violation in the $D$ meson system is expected to occur, but not at the level seen in the kaon system or expected in the $B$ meson system.
} 
Therefore, combining these two symmetries into one operation, the behaviour of the kaon and antikaon under the $C P$ symmetry becomes apparent:

$$
C P\left|K^{0}\right\rangle=-\left|\overline{K^{0}}\right\rangle, \quad C P\left|\overline{K^{0}}\right\rangle=-\left|K^{0}\right\rangle
$$

Equation 2.8 shows that $K^{0}$ and $\overline{K^{0}}$ are not good eigenstates of $C P$. For a state to be a "good" eigenstate of a symmetry operation, that state must be returned once the operation has been applied. For example, in order for a state $|\psi\rangle$ to be a good eigenstate of $C P$

$$
C P|\psi\rangle= \pm|\psi\rangle
$$

In other words, $C P$ affects the individual kaon states, leaving them different than they were initially. $C P$ symmetry applied to a kaon state returns an antikaon state, while $C P$ applied to the antikaon state returns a kaon state, which in both cases are not the same as the initial states.

However, because of the mixing property that kaons exhibit, where a kaon can change into its antiparticle, $K^{0} \rightleftharpoons \overline{K^{0}}$, the "particles" that are observed in the laboratory are not the individual kaon states $K^{0}$ and $\overline{K^{0}}$, but rather a linear combination of the two:

$$
\left|K_{1}^{0}\right\rangle=\frac{1}{\sqrt{2}}\left(\left|K^{0}\right\rangle-\left|\overline{K^{0}}\right\rangle\right), \quad\left|K_{2}^{0}\right\rangle=\frac{1}{\sqrt{2}}\left(\left|K^{0}\right\rangle+\left|\overline{K^{0}}\right\rangle\right)
$$

where the factor of $\frac{1}{\sqrt{2}}$ is included to normalize the states. In light of the fact that $K^{0}$ and $\overline{K^{0}}$ by themselves are not invariant under $C P$ symmetry, the $C P$ properties of these combined states will be considered. Using the previous results for the treatment of the individual kaon states $K^{0}$ and $\overline{K^{0}}$,

$$
\begin{aligned}
C P\left|K_{1}^{0}\right\rangle & =\frac{1}{\sqrt{2}}\left(C P\left|K^{0}\right\rangle-C P\left|\overline{K^{0}}\right\rangle\right) \\
& =\frac{1}{\sqrt{2}}\left(-\left|\overline{K^{0}}\right\rangle+\left|K^{0}\right\rangle\right) \\
& =\frac{1}{\sqrt{2}}\left(\left|K^{0}\right\rangle-\left|\overline{K^{0}}\right\rangle\right)
\end{aligned}
$$


which yields

$$
C P\left|K_{1}^{0}\right\rangle=+\left|K_{1}^{0}\right\rangle
$$

Similarly, for the second combination state, $K_{2}^{0}$,

$$
C P\left|K_{2}^{0}\right\rangle=-\left|K_{2}^{0}\right\rangle
$$

Each of the states $K_{1}^{0}$ and $K_{2}^{0}$ appear to be a good eigenstate of $C P$ because each satisfies the condition in Equation 2.9. Assuming that $C P$ is conserved in weak interactions, $K_{1}^{0}$ can only decay into a state having a $C P$ eigenvalue of +1 (even $C P$ symmetry), whereas $K_{2}^{0}$ must decay to a state having a $C P$ eigenvalue of -1 (odd $C P$ symmetry). The decay channel for neutral kaons is often into two or three pions. Since the two pion final state exhibits even $C P$ symmetry due to the negative intrinsic parity of each meson and the positive contribution from charge conjugation, it is associated with the decay of $K_{1}^{0}$. Correspondingly, $K_{2}^{0}$ decays to a three pion final state because the parity of three pion states gives odd $C P$ symmetry. Therefore, if weak interactions obey CP symmetry then $K_{1}^{0}$ will only decay into two pions, but never three, and $K_{2}^{0}$ will only decay into three pions, but never two:

$$
K_{1}^{0} \rightarrow 2 \pi, \quad K_{2}^{0} \rightarrow 3 \pi
$$

Of the two decays, the $2 \pi$ decay will take place much faster than that of the $3 \pi$ because the energy released is greater. Starting with a beam of $K^{0}$ particles, which can be thought of as being a mixture containing both $K_{1}^{0}$ and $K_{2}^{0}$, the $K_{1}^{0}$ component will quickly decay leaving only the $K_{2}^{0}$ component at some large distance along the beam. This is exactly the idea that was used to test $C P$ invariance. Given a long enough beamline ${ }^{3}$, the exclusion of $2 \pi$ final states at large distances as dictated by $C P$ symmetry can be quantitatively measured.

\footnotetext{
${ }^{3}$ The distance used in the experiment performed by Cronin and Fitch was 57 feet or $17 \mathrm{~m}$.
} 
In 1964 Cronin and Fitch [6] reported evidence that two pion final states were found at distances far down the beamline where only three pion final states were expected, giving unmistakable evidence of $C P$ violation. The assumption that $K_{1}^{0}$ and $K_{2}^{0}$ were perfect $C P$ eigenstates of $C P$ was not valid after all, and a small admixture of $K_{1}^{0}$ (ie., the "wrong" CP eigenstate) was needed to explain this unexpected presence of $2 \pi$ final states:

$$
\begin{aligned}
& \left|K_{L}^{0}\right\rangle=\frac{1}{1+|\epsilon|^{2}}\left(\left|K_{2}^{0}\right\rangle+\epsilon\left|K_{1}^{0}\right\rangle\right), \\
& \left|K_{S}^{0}\right\rangle=\frac{1}{1+|\epsilon|^{2}}\left(\left|K_{1}^{0}\right\rangle-\epsilon\left|K_{2}^{0}\right\rangle\right) .
\end{aligned}
$$

The labels L and S in Equations 2.14 and 2.15 refer to the lifetime of the particle"L" for long-lived and "S" for short-lived [2]:

$$
\begin{array}{ll}
K_{S}^{0} \rightarrow 2 \pi & \tau=0.89 \times 10^{-10} \mathrm{sec} \\
K_{L}^{0} \rightarrow 3 \pi & \tau=5.2 \times 10^{-8} \mathrm{sec} .
\end{array}
$$

The coefficient $\epsilon$ is introduced to characterize nature's departure from perfect $C P$ symmetry and is shown experimentally to be small, $\epsilon=2.3 \times 10^{-3}[2]$.

Further studies on $K_{L}^{0}$ revealed even more evidence for $C P$ violation. Violation of $C P$ symmetry gives rise to an observable difference in the branching ratios in a second $K_{L}^{0}$ decay mode:

$$
\begin{aligned}
& K_{L}^{0} \rightarrow \pi^{+}+e^{-}+\bar{\nu}_{e} \\
& K_{L}^{0} \rightarrow \pi^{-}+e^{+}+\nu_{e} .
\end{aligned}
$$

Experiments show that $K_{L}^{0}$ decays more often into a positron than an electron, by a small fractional amount $3.3 \times 10^{-3}[7]$, which means that this reaction is sensitive to the difference between matter and antimatter, producing more of one than the other. The fact that $C P$ violation allows unequal treatment of particles and antiparticles 
suggests a link to the matter-antimatter asymmetry in the universe. If the violation of $C P$ symmetry was built into the early universe in such a way that the production of matter was favoured over antimatter, then this might explain the matter dominance that apparently prevails throughout the observed universe today.

Even though $C P$ symmetry breaking was discovered 30 years ago, and many models exist to account for it, there is still no verified theory that has been shown to describe how $C P$ violation fits into the current theoretical models of particle physics. The goal of the SLAC B Factory is to make such a test using neutral $B$ mesons rather than neutral kaons to describe once and for all the source of $C P$ violation.

\section{3 $C P$ Violation in the Standard Model}

The current view of matter in the universe is that it consists of just two types of elementary particles: quarks and leptons. The theory describing the behaviour of these fundamental building blocks is known as the Standard Model of particle physics, which accounts for the electromagnetic force (describing the forces of electric and magnetic attraction and repulsion that bind atoms), the weak force (responsible for radioactive decay), and the strong nuclear force (which holds atomic nuclei together). So far, the Standard Model has been a very successful description of nature and to date, no experimental evidence has disagreed with its predictions. However, in order to incorporate $C P$ violation into the theoretical models of the 1950 s and later into the Standard Model, an addition to the theory was necessary, and it is this proposed addition that the $B_{A} B_{A R}$ group will be testing.

Quarks and leptons, the fundamental building blocks of the Standard Model, are found to exist in three patterns called families or generations. ${ }^{4}$ Table 2.1 shows the

\footnotetext{
${ }^{4}$ This is yet another mysterious facet of the Standard Model - why are there three families and not more or less? Moreover, why is more than one family required when all of the matter today is
} 
lepton and quark families explicitly. Each of the leptons and quarks listed below also has an associated antiparticle, which is oppositely charged.

\begin{tabular}{|c|c|c|c|}
\hline Generation & First & Second & Third \\
\hline \hline Leptons & electron, $e^{-}$ & muon, $\mu^{-}$ & tau, $\tau^{-}$ \\
& electron neutrino, $\nu_{e}$ & muon neutrino, $\nu_{\mu}$ & tau neutrino, $\nu_{\tau}$ \\
\hline Quarks & up, $u$ & charm, $c$ & top, $t$ \\
& down, $d$ & strange $s$ & bottom, $b$ \\
\hline
\end{tabular}

Table 2.1: Table showing the three generations of fundamental particles.

The existence of families gives rise to 4 of the free parameters of the Standard Model, namely the 4 parameters of the Cabibbo-Kobayashi-Maskawa (CKM) matrix that describe the mixing between quark generations. The quark mass eigenstates (the physical quarks) are not the same as the weak eigenstates, but they are related by a rotation matrix known as the CKM matrix ${ }^{5}$ :

$$
\left(\begin{array}{c}
d^{\prime} \\
s^{\prime} \\
b^{\prime}
\end{array}\right)_{w e a k}=\left(\begin{array}{lll}
V_{u d} & V_{u s} & V_{u b} \\
V_{c d} & V_{c s} & V_{c b} \\
V_{t d} & V_{t s} & V_{t b}
\end{array}\right)\left(\begin{array}{c}
d \\
s \\
b
\end{array}\right)_{p h y s i c a l} .
$$

By convention, the three quarks $u, c, t$ remain unmixed, while the $d, s, b$ quarks are mixed. The purpose of these matrix elements is to describe the coupling strength between quarks. For instance, $V_{u d}$ indicates the strength of the force between the $u$ and $d$ quarks, while $V_{u s}$ describes the coupling strength between the $u$ and $s$ quarks, and likewise for the other elements in the matrix.

composed of particles from the first generation alone? Scientists still do not know the answer to these questions.

${ }^{5}$ The idea of having to rotate quark states for the weak interaction is not at all obvious and was suggested by Cabibbo in 1963 in order to explain strangeness-changing weak processes. Assuming that a quark species cannot couple between generations, a strange quark is not supposed to be able to couple from the second generation with one from the first or third. However, in the decay of the lambda particle: $\Lambda \rightarrow p+\pi^{-}$, the strange quark that makes up part of the $\Lambda$ decays into an up quark on the right-hand side of the reaction. 
The CKM matrix elements are not all independent, and in the Standard Model of three generations, the nine elements of the CKM matrix are defined in terms of three real parameters (rotation angles) and one complex phase. This is shown explicitly in the following representation [2]:

$$
V_{\mathrm{CKM}}=\left(\begin{array}{ccc}
c_{12} c_{13} & s_{12} c_{13} & s_{13} e^{-i \delta_{13}} \\
-s_{12} c_{23}-c_{12} s_{23} s_{13} e^{i \delta_{13}} & c_{12} c_{23} c_{3}-s_{12} s_{23} s_{13} e^{i \delta_{13}} & s_{23} c_{13} \\
s_{12} s_{23}-c_{12} c_{23} s_{13} e^{i \delta_{13}} & -c_{12} s_{23}-s_{12} c_{23} s_{13} e^{i \delta_{13}} & c_{23} c_{13}
\end{array}\right)
$$

where $c_{i j}$ stands for $\cos \theta_{i j}$, and $s_{i j}$ for $\sin \theta_{i j}$ and where the indices $i, j$ are generation labels, $i, j=1,2,3$. In this form of the matrix, the presence of the three rotation angles $\left(\theta_{12}, \theta_{23}, \theta_{13}\right)$ and the phase angle $\left(\delta_{13}\right)$ is clear. A second possible parameterization that was suggested by Wolfenstein [8] has the following empirical form:

$$
V_{\mathrm{CKM}} \simeq\left(\begin{array}{ccc}
1-\lambda^{2} / 2 & \lambda & A \lambda^{3}(\rho-i \eta) \\
-\lambda & 1-\lambda^{2} / 2 & A \lambda^{2} \\
A \lambda^{3}(1-\rho-i \eta) & -A \lambda^{2} & 1
\end{array}\right)
$$

where $A, \lambda=\sin \theta_{\text {Cabibbo }} \approx 0.23$ and $\rho$ are the real parameters of the theory, and $\eta$ is the phase. A non-zero $\eta$ allows for the presence of $C P$ violation in the Standard Model. This particular form of the parameterization is correct up to terms of order $\lambda^{3}$. The Wolfenstein approximation is particularly instructive for understanding the structure of the matrix because the magnitudes of the elements are easily compared and the dependency on the real and imaginary parts is outlined clearly, with the phase present in only two of the nine elements.

The only constraint the Standard Model places on the CKM matrix is that it must be unitary; that is, it must satisfy the property: $V V^{\dagger}=V^{\dagger} V=1$. Written as matrices, this constraint becomes:

$$
V^{\dagger} V=\left(\begin{array}{ccc}
V_{u d}^{*} & V_{c d}^{*} & V_{t d}^{*} \\
V_{u s}^{*} & V_{c s}^{*} & V_{t s}^{*} \\
V_{u b}^{*} & V_{c b}^{*} & V_{t b}^{*}
\end{array}\right)\left(\begin{array}{ccc}
V_{u d} & V_{u s} & V_{u b} \\
V_{c d} & V_{c s} & V_{c b} \\
V_{t d} & V_{t s} & V_{t b}
\end{array}\right)=\left(\begin{array}{ccc}
1 & 0 & 0 \\
0 & 1 & 0 \\
0 & 0 & 1
\end{array}\right)
$$


Multiplying the the third row of $V^{\dagger}$ with the first column of $V$ gives rise to the following relationship between six of the CKM matrix elements:

$$
V_{u b}^{*} V_{u d}+V_{c b}^{*} V_{c d}+V_{t b}^{*} V_{t d}=0
$$

There are other similar equations (six to be exact, combining three rows and three columns), but Equation 2.23 in the Wolfenstein parameterization contains the most significant imaginary parts and is therefore the most interesting for $C P$ violation because it is the equation most sensitive to the complex phase.

In a particular representation in which one of the terms in Equation 2.23 is real, the unitarity constraint can be visualized geometrically as a triangle in the complex plane, the sides of which are formed by the complex quantities $V_{u b}^{*} V_{u d}, V_{t b}^{*} V_{t d}$ while the base has length $V_{c b}^{*} V_{c d}$, as shown in Figure 2.4. The triangle depicted in Figure 2.4 is usually referred to as the unitarity triangle since it represents one of the constraints obtained from a unitary quark mixing matrix.

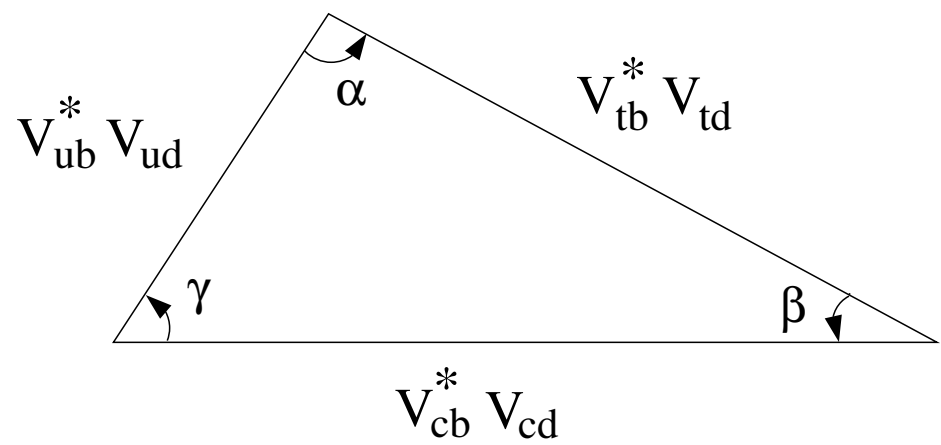

Figure 2.4: Shown is the standard parameterization of the unitarity triangle as visualized in the complex plane. It represents one of the unitarity constraints obtained from having a unitary quark mixing matrix and is formed by the complex matrix elements $V_{u b}^{*} V_{u d}, V_{t b}^{*} V_{t d}$ and $V_{c b}^{*} V_{c d}$.

An arbitrary point in the complex plane can be written as $z=r e^{i \theta}$, where $\theta$ is the angle to the real axis and is called the argument of $z$. Using this terminology, the 
interior angles of the unitarity triangle, labeled $\alpha, \beta$ and $\gamma$ as shown in Figure 2.4, are related to CKM matrix elements in the following manner [9].

$$
\begin{aligned}
& \alpha \equiv \arg \left(\frac{V_{t d} V_{t b}^{*}}{V_{u d} V_{u b}^{*}}\right), \\
& \beta \equiv \arg \left(\frac{V_{c d} V_{c b}^{*}}{V_{t d} V_{t b}^{*}}\right), \\
& \gamma \equiv \arg \left(\frac{V_{u d} V_{u b}^{*}}{V_{c d} V_{c b}^{*}}\right) .
\end{aligned}
$$

Rescaling the three sides of the triangle in Figure 2.4 by the length of the base, $V_{c b}^{*} V_{c d}$, the coordinates of the three vertices of the triangle in the complex plane are then given by $(0,0),(1,0)$ and $(\rho, \eta)$ where

$$
\rho=\operatorname{Re}\left(\frac{V_{u b}^{*} V_{u d}}{V_{c b}^{*} V_{c d}}\right) \quad \text { and } \quad \eta=\operatorname{Im}\left(\frac{V_{u b}^{*} V_{u d}}{V_{c b}^{*} V_{c d}}\right)
$$

Recall that $\eta$ is the phase expected to give rise to $C P$ violation within the Standard Model. Measuring any one of the angles of the unitarity triangle to be non-zero immediately results in $C P$ violation.

Measurements of $C P$ asymmetries will determine the interior angles of the unitarity triangle, which in turn will lead to information about the CKM matrix elements that give rise to the angles. The magnitude of the matrix element $V_{u d}$ is already well measured, and the magnitudes of $V_{t d}$ and $V_{t b}$ are well constrained in the Standard Model assumption that there are only three quark generations. The remaining quantity, the magnitude of $V_{c b}$, is presently being studied at CLEO II and LEP I with possible improvement from $B_{A} B A R$.

As discussed in Section 2.2, $C P$ violation has already been measured in the neutral kaon system, but in kaon experiments it is very difficult to determine whether the complex phase introduced in the CKM matrix is the only source for the observed 


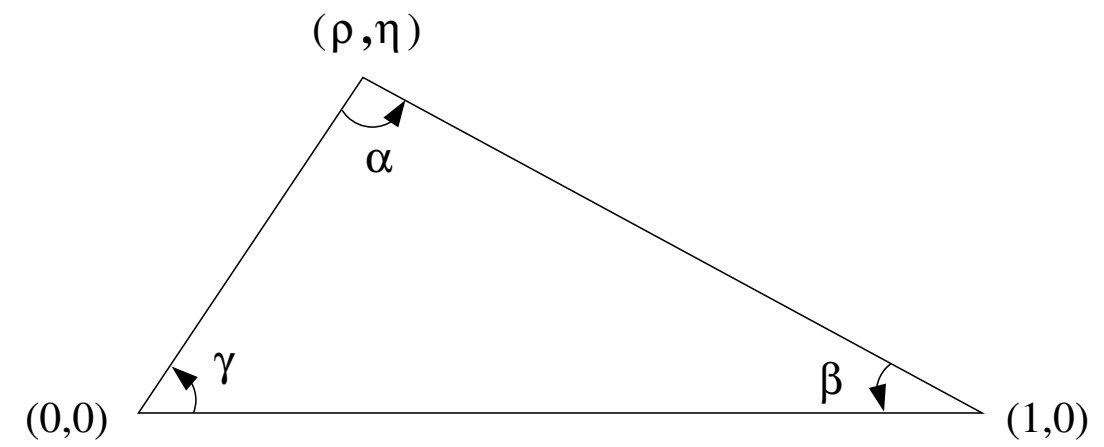

Figure 2.5: Shown is the unitarity triangle as visualized in the complex plane which represents one of the unitarity constraints obtained from having a unitary quark mixing matrix. This particular parameterization is normalized so that the base has unit length. The quantities describing the apex of the triangle, $(\rho, \eta)$, both depend on CKM matrix elements as shown in Equation 2.27.

asymmetries. Neutral $B$ meson decays however are not expected to have these ambiguities. Moreover, $C P$ asymmetries are predicted to be larger than those in kaon system, so experiments involving $B$ mesons are expected to provide the first precise measurement of the complex CKM phase. The aim of the $B_{A} B_{A R}$ group is to measure at least two angles and two sides of the unitarity triangle in order to overconstrain the $C P$ parameters.

\section{4 $C P$ Violation in the B Meson System}

In the treatment ${ }^{6}$ that follows, $C P$ violation in the $B$ meson system will be examined at a fairly detailed level. It will be shown that $C P$ violation in the $B$ meson system manifests itself in the form of interference in decay amplitudes which leads to an asymmetry in the decay rates of the $B^{0}$ and $\overline{B^{0}}$. The hope is to demonstrate quite clearly

\footnotetext{
${ }^{6}$ The conference proceedings by P.R. Burchat [10] is an especially useful reference for this section. The treatment that follows outlines the main aspects of $C P$ violation in the $B$ meson system presented in those proceedings.
} 
how this asymmetry is developed and what decay modes will be used to study it.

\subsubsection{The Physical $B$ Mesons}

Neutral $B$ mesons $\left(B^{0}=\bar{b} d\right.$ and $\left.\overline{B^{0}}=b \bar{d}\right)$ are another set of special particles like the kaon that have distinct antiparticle states, and where the particle and antiparticle can mix back and forth into one another, $B^{0} \rightleftharpoons \overline{B^{0}}$. Figure 2.6 shows the box diagram which illustrates the second order weak process that allows the $B$ meson to decay into its antiparticle and vice versa.

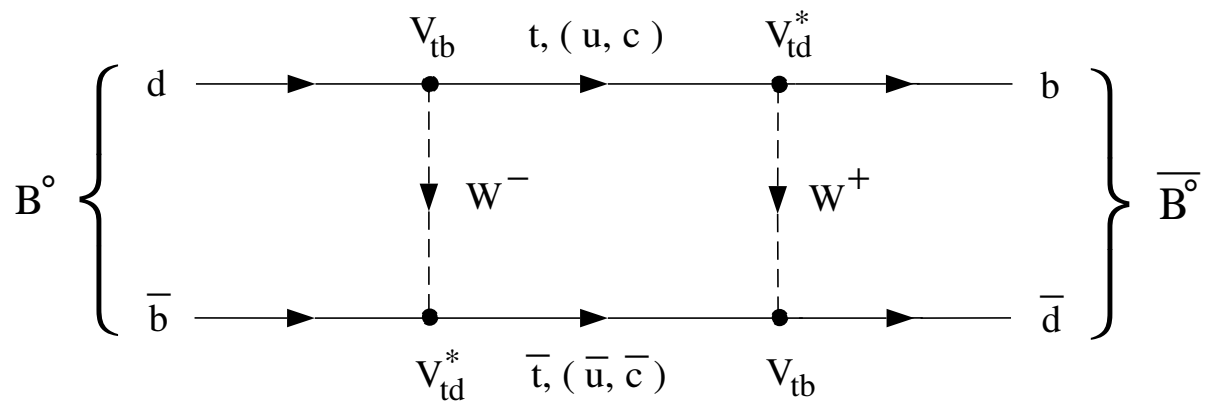

Figure 2.6: Box diagram for $B$ meson system showing how a $B^{0}$ particle can transform into its antiparticle. While other diagrams involving the $u$ and $c$ quarks contribute to the amplitude for the process, the diagram with the $t$ quark is expected to dominate.

Also analogous to the neutral kaons, neutral $B$ mesons produced at time $t=0$ are not necessarily states of definite mass (the states seen in the lab as time progresses), but rather some linear combination of states $a\left|B^{0}\right\rangle+b\left|\overline{B^{0}}\right\rangle$, similar to $K_{1}^{0}$ and $K_{2}^{0}$ (Equation 2.10) in the kaon system. Such a state is governed by the time-dependent Schrödinger equation, which is written as:

$$
i \frac{d}{d t}\left(\begin{array}{l}
a \\
b
\end{array}\right)=H\left(\begin{array}{l}
a \\
b
\end{array}\right)
$$


where the Hamiltonian, $H$, can be written in the form

$$
H=\left(\begin{array}{cc}
M & M_{12} \\
M_{12}^{*} & M
\end{array}\right)-\frac{i}{2}\left(\begin{array}{cc}
\Gamma & \Gamma_{12} \\
\Gamma_{12}^{*} & \Gamma
\end{array}\right) .
$$

The first matrix in Equation 2.29 is the mass matrix which describes processes which have virtual intermediate states, not those that lead to the decay of the meson. In the mass matrix, the off-diagonal elements $M_{12}$ are due to $B^{0} \rightarrow \overline{B^{0}}$ transitions with virtual intermediate states. The box diagram shown in Figure 2.6 indicates that the interaction can take place using all of the three possible up-type quarks as the virtual state: the up quark, charm quark or top quark. The diagram involving the top quark dominates, however, because in the renormalization of the box diagram, there are factors in the expression for the amplitude which depend on the square of the quark masses as well as the square of the appropriate CKM couplings [11]:

$$
M_{12} \propto G_{F}^{2}\left[m_{c}^{2} \xi_{c}^{2}+m_{t}^{2} \xi_{t}^{2}+2 m_{c}^{2} \xi_{c} \xi_{t} \ln \frac{m_{t}^{2}}{m_{c}^{2}}\right]
$$

where $\xi_{i} \equiv V_{i d}^{*} V_{i s}$ for kaons, and $\xi_{i} \equiv V_{i d}^{*} V_{i s}$ in the case of the $B$ meson. This form of $M_{12}$ also assumes that the mass of the top quark is small in comparison with the mass of the mediating $W$ boson, which is not true. However, Equation 2.30 is meant to illustrate the relative contributions from the charm and top quark loops instead of being rigorously exact. Due to its small mass ${ }^{7}$, the contribution of the up quark is very small in both the kaon and $B$ meson diagrams and is not included in Equation 2.30. In the case of the $B$ meson, the top quark dominates because it happens to be the heaviest by far of the three quark choices in addition to contributing sizable CKM coupling factors. Therefore, Equation 2.30 demonstrates that the $M_{12}$ matrix element is proportional to the CKM quark mixing elements involving the top quark found at

\footnotetext{
${ }^{7}$ The bare mass of the up quark is $\approx 4.2 \mathrm{MeV}$, whereas the mass of the charm quark is $\approx 1.1 \mathrm{GeV}$, and the mass of the top quark is $\approx 175 \mathrm{GeV}$. Therefore, the mass of the charm is about $10^{3}$ times smaller than that of the charm quark and $10^{5}$ times smaller than the top quark.
} 
the four vertices of the box diagram:

$$
M_{12} \propto\left(V_{t b} V_{t d}^{*}\right)^{2}
$$

The second matrix in Equation 2.29 is the decay matrix and is due to processes with real intermediate states which can lead to the exponential decay of the system. In the decay matrix, the off-diagonal elements $\Gamma_{12}$ describe transitions from $B^{0}$ to $\overline{B^{0}}$ that have real intermediate states that are accessible to both $B^{0}$ and $\overline{B^{0}}$. However, $B^{0}$ and $\overline{B^{0}}$ have very few intermediate states in common, and as a result, $\Gamma_{12}$ is expected to be very small. In particular, it is expected to be to be much smaller than $M_{12}$ [9]:

$$
\left|\Gamma_{12}\right| \ll\left|M_{12}\right|
$$

This differs from the kaon system where $\left|\Gamma_{12}\right|$ is the larger term by far because $K^{0}$ and $\overline{K^{0}}$ have dominant decay modes in common. For example, the decay channels $K \rightarrow$ $2 \pi$ and $K \rightarrow 3 \pi$ mentioned in Section 2.2 are available to both the $K^{0}$ and $\overline{K^{0}}$.

Now that some of the general formalism has been established, consider the states of definite mass for the $B$ meson system, $B_{1}$ and $B_{2}$ :

$$
B_{1}=p\left|B^{0}\right\rangle+q\left|\overline{B^{0}}\right\rangle, \quad B_{2}=p\left|B^{0}\right\rangle-q\left|\overline{B^{0}}\right\rangle
$$

where the coefficients $p$ and $q$ obey the normalization:

$$
|p|^{2}+|q|^{2}=1
$$

and are related in the following way:

$$
\frac{q}{p}=\sqrt{\frac{M_{12}^{*}-i \Gamma_{12}^{*} / 2}{M_{12}-i \Gamma_{12} / 2}}
$$

which, using the approximation of Equation 2.32, can be written as:

$$
\frac{q}{p} \approx \sqrt{\frac{M_{12}^{*}}{M_{12}}} .
$$


Alternatively, the approximation in Equation 2.36 can be expressed in the following form:

$$
\left|\frac{q}{p}\right| \approx 1
$$

The eigenvalues of $H$ corresponding to the time-dependent eigenvectors $B_{1}$ and $B_{2}$ are $\left(m_{1}-\frac{i}{2} \Gamma_{1}\right)$ and $\left(m_{2}-\frac{i}{2} \Gamma_{2}\right)$ such that:

$$
i \frac{d}{d t} B_{j}=\left(m_{j}-\frac{i}{2} \Gamma_{j}\right) B_{j}, \quad j=1,2
$$

where the quantities $m_{1,2}$ and $\Gamma_{1,2}$ are given by

$$
\begin{array}{cc}
m_{1} \equiv M+\operatorname{Re} Q, & m_{2} \equiv M-\operatorname{Re} Q, \\
\Gamma_{1} \equiv \Gamma+\operatorname{Im} Q, & \Gamma_{2} \equiv \Gamma-\operatorname{Im} Q
\end{array}
$$

and

$$
Q \equiv \sqrt{\left(M_{12}^{*}-\frac{i}{2} \Gamma_{12}^{*}\right)\left(M_{12}-\frac{i}{2} \Gamma_{12}\right)}
$$

In general, the prescription for obtaining a time-dependent state, $B(t)$, from the state at time $t=0, B(0)$, is given as [12]:

$$
B(\mathbf{x}, t)=e^{-i H t} B(\mathbf{x}, 0)
$$

By virtue of the fact that $B(\mathbf{x}, 0)$ is an eigenfunction of $H$, the solution to the time dependent Schrödinger equation (Equation 2.38) can be written in terms of the Hamiltonian and the state $B$ at $t=0$ which is a constant with respect to time:

$$
B_{j}(\mathbf{x}, t)=B_{j}(\mathbf{x}, 0) e^{-i m_{j} t} e^{-\Gamma_{j} t / 2}, \quad j=1,2
$$

which describes a physical particle with mass $m_{j}$ and decay rate $\Gamma_{j}$. 


\subsubsection{Time Evolution of the Physical State}

When a neutral $B$ meson is produced (for example, in an $e^{+} e^{-}$collision), it starts off as a pure $B^{0}$ or $\overline{B^{0}}$ meson. However, the initially pure state $B^{0}$ develops a $\overline{B^{0}}$ component in time through mixing as shown in Figure 2.6. Therefore, the state of the $B$ meson as a function of time will be a mixture of the two eigenstates $B^{0}$ and $\overline{B^{0}}$. Using Equation 2.33, it is simple to show that $B^{0}$ and $\overline{B^{0}}$ can be written in terms of $B_{1}$ and $B_{2}$ as follows:

$$
\left|B_{\text {phys }}^{0}(t)\right\rangle=\frac{\left|B_{1}\right\rangle+\left|B_{2}\right\rangle}{2 p} \quad \text { and } \quad\left|\bar{B}_{\text {phys }}(t)\right\rangle=\frac{\left|B_{1}\right\rangle-\left|B_{2}\right\rangle}{2 q} .
$$

Furthermore, Equation 2.43 can be used to cast the expressions for $B^{0}$ and $\overline{B^{0}}$ in a slightly different manner. For now, consider only $\left|B_{\text {phys }}^{0}(t)\right\rangle$ :

$$
\left|B_{\text {phys }}^{0}(t)\right\rangle=\frac{1}{2 p}\left\{\left[\left|B_{1}(0)\right\rangle e^{-i m_{1} t} e^{-\Gamma_{1} / 2}\right]+\left[\left|B_{2}(0)\right\rangle e^{-i m_{2} t} e^{-\Gamma_{2} / 2}\right]\right\} .
$$

The states at $t=0,\left|B_{1}(0)\right\rangle$ and $\left|B_{2}(0)\right\rangle$, can be evaluated by applying Equation 2.33 once again:

$$
\left|B_{\text {phys }}^{0}(t)\right\rangle=\frac{1}{2 p}\left[\left(p\left|B^{0}\right\rangle+q\left|\overline{B^{0}}\right\rangle\right) e^{-i m_{1} t} e^{-\Gamma_{1} / 2}+\left(p\left|B^{0}\right\rangle-q\left|\overline{B^{0}}\right\rangle\right) e^{-i m_{2} t} e^{-\Gamma_{2} / 2}\right] .
$$

Assuming that the decay rates $\Gamma_{1}$ and $\Gamma_{2}$ are comparable [9]:

$$
\Gamma_{1}=\Gamma_{2} \equiv \Gamma
$$

the expression in Equation 2.46 can be written as:

$$
\begin{aligned}
&\left|B_{\text {phys }}^{0}(t)\right\rangle=\frac{1}{2 p} e^{-i\left(\frac{m_{1}+m_{2}}{2}\right) t} e^{-\Gamma t / 2}\left\{p\left|B^{0}\right\rangle\left[e^{-i\left(\frac{m_{1}-m_{2}}{2}\right) t}+e^{-i\left(\frac{m_{1}-m_{2}}{2}\right) t}\right]\right. \\
&\left.+q\left|\overline{B^{0}}\right\rangle\left[e^{-i\left(\frac{m_{1}-m_{2}}{2}\right) t}-e^{-i\left(\frac{m_{1}-m_{2}}{2}\right) t}\right]\right\} .
\end{aligned}
$$

Finally, the following results from complex analysis [13]:

$$
\text { (i) } \cos \theta=\frac{e^{i \theta}+e^{-i \theta}}{2}, \quad \text { (ii) } \sin \theta=\frac{e^{i \theta}-e^{-i \theta}}{2 i}
$$


can be used to give the following superposition of weak eigenstates describing the time evolution of an initially pure $B^{0}$ state:

$$
\left|B_{\text {phys }}^{0}(t)\right\rangle=f_{+}(t)\left|B^{0}\right\rangle+\frac{q}{p} f_{-}(t)\left|\overline{B^{0}}\right\rangle
$$

where the quantities $f_{+}$and $f_{-}$are defined as follows:

$$
f_{+} \equiv e^{-i m t} e^{-\Gamma t / 2} \cos \left(\frac{\Delta m t}{2}\right)
$$

and

$$
f_{-} \equiv e^{-i m t} e^{-\Gamma t / 2} i \sin \left(\frac{\Delta m t}{2}\right)
$$

In the above equations, $\Delta m$ is the mass difference between the two physical states and $m$ is the mean mass:

$$
\Delta m \equiv m_{1}-m_{2}, \quad m \equiv \frac{m_{1}+m_{2}}{2}
$$

A similar expression is obtained for the time dependent system that evolves from an initially pure $\overline{B^{0}}$ state:

$$
\left|{\overline{B^{0}}}_{\text {phys }}(t)\right\rangle=\frac{q}{p} f_{-}(t)\left|B^{0}\right\rangle+f_{+}(t)\left|\overline{B^{0}}\right\rangle
$$

\subsubsection{Decays to $C P$ Eigenstates}

Now, consider the probability of decay or amplitude for an initially pure $B^{0}$ or $\overline{B^{0}}$ to decay to a final state at time $t$, where the final state is a $C P$ eigenstate labeled as $f_{C P}$. Recall that for a state to be a $C P$ eigenstate, the following condition must be met:

$$
C P\left|f_{C P}\right\rangle= \pm\left|f_{C P}\right\rangle
$$

and that these final states are accessible to both $B^{0}$ and $\overline{B^{0}}$ decays. The amplitudes for a $B^{0}$ or $\overline{B^{0}}$ to decay to a $C P$ eigenstate, $f_{C P}$, are obtained by contracting Equations 2.50 
and 2.54 on the left by the final state $\left\langle f_{C P}\right|$ to obtain

$$
\left\langle f_{C P} \mid B_{\text {phys }}^{0}(t)\right\rangle=f_{+}(t)\left\langle f_{C P} \mid B^{0}\right\rangle+\frac{q}{p} f_{-}(t)\left\langle f_{C P} \mid \overline{B^{0}}\right\rangle
$$

and

$$
\left\langle f_{C P} \mid{\overline{B^{0}}}_{p h y s}(t)\right\rangle=\frac{p}{q} f_{-}(t)\left\langle f_{C P} \mid B^{0}\right\rangle+f_{+}(t)\left\langle f_{C P} \mid \overline{B^{0}}\right\rangle
$$

respectively.

For convenience, the following definitions will be made:

$$
A \equiv\left\langle f_{C P} \mid B^{0}\right\rangle, \quad \bar{A} \equiv\left\langle f_{C P} \mid \overline{B^{0}}\right\rangle
$$

and

$$
\lambda \equiv \frac{q}{p} \frac{\left\langle f_{C P} \mid \overline{B^{0}}\right\rangle}{\left\langle f_{C P} \mid B^{0}\right\rangle}=\frac{q}{p} \bar{A} .
$$

The first amplitude, $A$, describes the probability for the $B^{0}$ system to decay into a final state $f_{C P}$, while the second amplitude, $\bar{A}$, describes the probability for the $\overline{B^{0}}$ system to decay into a final state $f_{C P}$. The defined quantity $\lambda$ therefore depends on the mixing quantities $q$ and $p$, as well as the decay amplitudes. Using Equation 2.36, $\lambda$ can be approximated as:

$$
\lambda \approx \sqrt{\frac{M_{12}^{*}}{M_{12}}} \frac{\bar{A}}{A} .
$$

The previous expressions for the decay amplitudes can then be written in a simple manner using the definitions for $A, \bar{A}$, and $\lambda$. Equations 2.56 becomes:

$$
\left\langle f_{C P} \mid B_{\text {phys }}^{0}(t)\right\rangle=A\left[f_{+}(t)+\lambda f_{-}(t)\right],
$$

and Equation 2.57 can be written as:

$$
\left\langle f_{C P} \mid \overline{B^{0}}{ }_{p h y s}(t)\right\rangle=A \frac{p}{q}\left[f_{-}(t)+\lambda f_{+}(t)\right]
$$

or,

$$
\left\langle f_{C P} \mid{\overline{B^{0}}}_{p h y s}(t)\right\rangle=\bar{A}\left[f_{-}(t)+\lambda^{-1} f_{+}(t)\right] .
$$


The decay rate, $\Gamma$, for a given transition is governed by the product of the amplitude and its complex conjugate:

$$
\Gamma=\mid \text { amplitude }\left.\right|^{2}=(\text { amplitude })(\text { amplitude })^{*}
$$

Therefore, in the case of $B_{p h y s}^{0}(t) \rightarrow f_{C P}$, multiplying Equation 2.61 together with its complex conjugate yields the following expression for the time-dependent rate:

$$
\Gamma\left(B_{\text {phys }}^{0}(t) \rightarrow f_{C P}\right)=|A|^{2}\left[\left|f_{+}\right|^{2}+|\lambda|^{2}\left|f_{-}\right|^{2}+\lambda^{*} f_{+} f_{-}+\lambda f_{-} f_{+}\right] .
$$

Using the definitions of $f_{+}$and $f_{-}$from Equations 2.50 and 2.54, the above expression becomes:

$$
\begin{aligned}
\Gamma\left(B_{\text {phys }}^{0}(t) \rightarrow f_{C P}\right)=|A|^{2} e^{-\Gamma t} & {\left[\cos ^{2}\left(\frac{\Delta m t}{2}\right)+|\lambda|^{2} \sin ^{2}\left(\frac{\Delta m t}{2}\right)\right.} \\
& -i \lambda \cos \left(\frac{\Delta m t}{2}\right) \sin \left(\frac{\Delta m t}{2}\right) \\
& \left.+i \lambda \sin \left(\frac{\Delta m t}{2}\right) \cos \left(\frac{\Delta m t}{2}\right)\right] .
\end{aligned}
$$

Using the identity from complex analysis that states [13]:

$$
\operatorname{Im} z=\frac{z-z^{*}}{2 i}
$$

the expression for the time-dependent rate can be written as:

$$
\begin{aligned}
\Gamma\left(B_{\text {phys }}^{0}(t) \rightarrow f_{C P}\right)= & |A|^{2} e^{-\Gamma t}\left[\cos ^{2}\left(\frac{\Delta m t}{2}\right)+|\lambda|^{2} \sin ^{2}\left(\frac{\Delta m t}{2}\right)\right. \\
& \left.-2 \operatorname{Im} \lambda \sin \left(\frac{\Delta m t}{2}\right) \cos \left(\frac{\Delta m t}{2}\right)\right]
\end{aligned}
$$

Finally, using the trigonometric identities:

$$
\text { (i) } \sin ^{2} \theta=\frac{1}{2}(1-\cos 2 \theta), \quad(i i) \cos ^{2} \theta=\frac{1}{2}(1+\cos 2 \theta),
$$

the decay rate takes the following form:

$$
\Gamma\left(B_{\text {phys }}^{0}(t) \rightarrow f_{C P}\right)=|A|^{2} e^{-\Gamma t}\left[\frac{1+|\lambda|^{2}}{2}+\frac{1-|\lambda|^{2}}{2} \cos (\Delta m t)-\operatorname{Im} \lambda \sin (\Delta m t)\right] .
$$


In the case of $\bar{B}_{\text {phys }}(t) \rightarrow f_{C P}$, a similar relationship is obtained for the rate except that $\lambda$ is replaced by $\lambda^{-1}$, as seen when comparing Equation 2.62 to Equation 2.61:

$\Gamma\left({\overline{B^{0}}}_{\text {phys }}(t) \rightarrow f_{C P}\right)=|A|^{2} e^{-\Gamma t}\left[\frac{1+|\lambda|^{2}}{2}+\frac{1-|\lambda|^{2}}{2} \cos (\Delta m t)+\operatorname{Im} \lambda \sin (\Delta m t)\right]$

The cross-term in both of the above equations (the term including the $\operatorname{Im} \lambda$ factor) represents interference in the decay, and will eventually be shown to be the source of $C P$ violation in the $B$ meson system.

The time-dependent $C P$ asymmetry, $a_{f_{C P}}(t)$, which is the difference in the decay rate of an initially pure $B^{0}$ state to a $C P$ eigenstate as compared to the decay rate of an initially pure $\overline{B^{0}}$ state to a $C P$ eigenstate, is defined as follows:

$$
a_{f_{C P}}(t) \equiv \frac{\Gamma\left[B_{\text {phys }}^{0}(t) \rightarrow f_{C P}\right]-\Gamma\left[\overline{B^{0}}{ }_{\text {phys }}(t) \rightarrow f_{C P}\right]}{\Gamma\left[B_{\text {phys }}^{0}(t) \rightarrow f_{C P}\right]+\Gamma\left[\bar{B}^{0}{ }_{\text {phys }}(t) \rightarrow f_{C P}\right]}
$$

where the denominator is included simply to normalize $a_{f_{C P}}$. Substituting Equations 2.69 and 2.70 into the definition for the decay rate asymmetry results in the following expression:

$$
a_{f_{C P}}(t)=\frac{\left(1-|\lambda|^{2}\right) \cos (\Delta m t)-2 \operatorname{Im} \lambda \sin (\Delta m t)}{1+|\lambda|^{2}}
$$

Solving for $\operatorname{Im} \lambda$ in Equation 2.72 gives information about the corresponding CKM elements and therefore the phase.

To simplify Equation 2.72 consider the special case where $|\bar{A} / A| \simeq 1$ :

$$
\left|\frac{\bar{A}}{A}\right|=\left|\frac{\left\langle f_{C P} \mid \overline{B^{0}}\right\rangle}{\left\langle f_{C P} \mid B^{0}\right\rangle}\right| \simeq 1
$$

which is justified because the same combination of quark mixing elements will contribute both in the case $B_{\text {phys }}^{0}(t) \rightarrow f_{C P}$ and $\bar{B}_{p h y s}(t) \rightarrow f_{C P}$. Using Equation 2.73 in conjunction with the approximation for $|q / p|$ found in Equation 2.37, $\lambda$ can be 
expressed simply as:

$$
|\lambda|=\left|\frac{q}{p}\right|\left|\frac{\bar{A}}{A}\right| \approx 1
$$

which means that $\operatorname{Im} \lambda$ can be written

$$
\operatorname{Im} \lambda=-\operatorname{Im} \lambda^{-1}
$$

Applying relations 2.74 and 2.75 in conjunction with two trigonometric identities:

$$
\text { (i) } \sin ^{2} \theta+\cos ^{2} \theta=1, \quad \text { (ii) } 2 \sin \theta \cos \theta=\sin 2 \theta \text {, }
$$

the expressions for the decay rates in Equations 2.69 and 2.70 become much simpler:

$$
\Gamma\left(B_{\text {phys }}^{0}(t) \rightarrow f_{C P}\right)=|A|^{2} e^{-\Gamma t}[1-\operatorname{Im} \lambda \sin (\Delta m t)]
$$

and

$$
\Gamma\left(\bar{B}_{\text {phys }}(t) \rightarrow f_{C P}\right)=|A|^{2} e^{-\Gamma t}[1+\operatorname{Im} \lambda \sin (\Delta m t)]
$$

Notice that the transitions of $B_{\text {phys }}^{0}(t) \rightarrow f_{C P}$ and ${\overline{B^{0}}}_{\text {phys }}(t) \rightarrow f_{C P}$ depend on the probability of decay, $|A|^{2}$, and the exponential decay, $e^{-\Gamma t}$, but also on an additional factor, $[1 \pm \operatorname{Im} \lambda \sin (\Delta m t)]$, which is different in each case. That is, the decay rate for a state that begins as a pure $B^{0}$ initially is not the same as the rate for a state that begins as a $\overline{B^{0}}$ state provided the $[1 \pm \operatorname{Im} \lambda \sin (\Delta m t)]$ factor is non-zero.

The time-dependent $C P$ asymmetry, $a_{f_{C P}}(t)$, is then given by the simplified expression:

$$
a_{f_{C P}}(t) \equiv \frac{\Gamma\left[B_{\text {phys }}^{0}(t) \rightarrow f_{C P}\right]-\Gamma\left[{\overline{B^{0}}}_{\text {phys }}(t) \rightarrow f_{C P}\right]}{\Gamma\left[B_{\text {phys }}^{0}(t) \rightarrow f_{C P}\right]+\Gamma\left[{\overline{B^{0}}}_{p h y s}(t) \rightarrow f_{C P}\right]} \approx-\operatorname{Im} \lambda \sin (\Delta m t) .
$$

The asymmetry in the decay rates is directly proportional to the quantity $\operatorname{Im} \lambda$ which can in turn be related to CKM matrix elements and the angles of the unitarity triangle, as well as the quantities $\Delta m$ which is known and $t$ which will be measured experimentally. Therefore measuring the asymmetry $a_{f_{C P}}(t)$ will allow for the isolation of the CKM elements containing the phase. 
The above treatment has shown that $C P$ violation in the neutral $B$ system arises from the interference between the amplitude for direct decay and the amplitude for the decay proceeding through particle-antiparticle mixing. Figure 2.7 illustrates this effect by emphasizing how two separate decay routes reach the same final $C P$ state. As is explicitly shown in Equation 2.79, the interference has different magnitudes depending on whether the intial state was a $B^{0}$ or a $\overline{B^{0}}$.

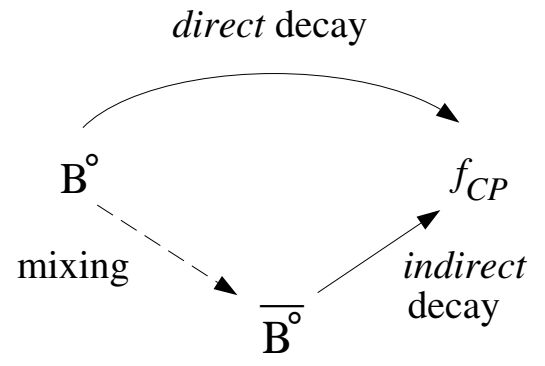

(a)

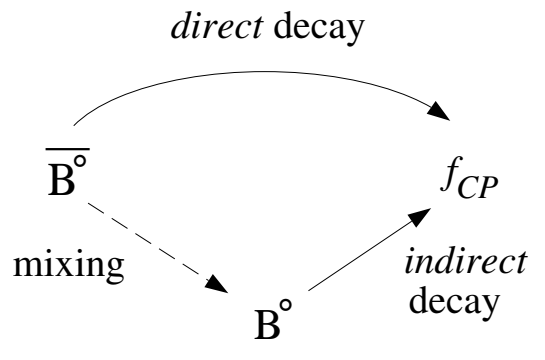

(b)

Figure 2.7: Shown is an illustration of how $C P$ violation in the neutral $B$ meson system results from the interference between the amplitude for direct decay and the amplitude for indirect decay that proceeds through mixing. The interference has different magnitudes depending on whether the initial state was (a) a $B^{0}$ particle or (b) a $\overline{B^{0}}$ particle.

\subsection{Decay Modes that Measure the Angles of the Unitarity Triangle}

The task now is to find decay reactions to $C P$ eigenstates that involve the appropriate CKM matrix elements. $B^{0}$ decays are obvious candidates because they involve the $b$ quark process that is required to give information about the matrix element $V_{u b}$. Three $B$ meson decay modes that will be considered are (i) $B^{0} \rightarrow \pi^{+} \pi^{-}$, (ii) $B^{0} \rightarrow J / \psi K_{S}^{0}$, and (iii) $B_{s}^{0} \rightarrow \rho K_{S}^{0}$. The first two decays use a $B^{0}$ meson which incorporates a $\bar{b}$ and 
$d$ quark, whereas in the third decay, $b$ quark processes are studied using $B_{s}^{0}$ mesons which are made of a $\bar{b}$ and $s$ quark. Because these decays are CKM suppressed, the probability of decay or branching ratio in each case is very small, on the order of $10^{-5}$. The fact that the processes under consideration are rare decays will have a strong influence on the experiment which will be discussed in the next chapter.

\subsubsection{The decay $B^{0} \rightarrow \pi^{+} \pi^{-}$}

The Feynman diagram for the decay $B^{0} \rightarrow \pi^{+} \pi^{-}$is given in Figure 2.8, where it is evident that the amplitude, $\left\langle f_{C P} \mid B^{0}\right\rangle$ depends on the CKM matrix elements $V_{u b}^{*}$ and $V_{u d}$.

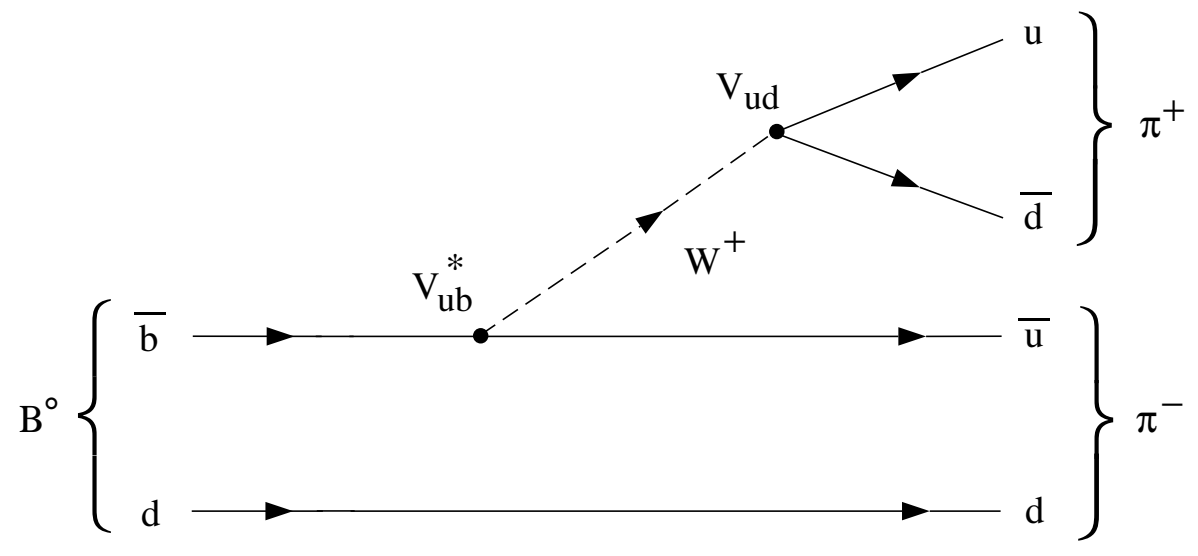

Figure 2.8: Feynman diagram for the decay $B^{0} \rightarrow \pi^{+} \pi^{-}$. The amplitude for this process will depend on the CKM matrix elements $V_{u b}$ and $V_{u d}$. Recall that in the Wolfenstein parameterization, $V_{u b}$ is expected to depend on the complex phase.

Using Equation 2.60, the connection between $\operatorname{Im} \lambda$ and the quark mixing elements of the CKM matrix is established by recalling that since the mass matrix element, $M_{12}$, from the Hamiltonian describes $B^{0} \rightarrow \overline{B^{0}}$ mixing (Figure 2.6), it will have the following 
dependence:

$$
M_{12} \propto\left(V_{t d}^{*} V_{t b}\right)^{2}
$$

Therefore, the expression for $\lambda$ becomes:

$$
\begin{aligned}
\lambda_{B^{0} \rightarrow \pi^{+} \pi^{-}} & =\sqrt{\frac{M_{12}^{*}}{M_{12}}} \frac{\bar{A}}{A} \\
& =\left(\frac{V_{t b}^{*} V_{t d}}{V_{t b} V_{t d}^{*}}\right)_{B \text { mixing }}\left(\frac{V_{u b} V_{u d}^{*}}{V_{u b}^{*} V_{u d}}\right)_{B \text { decay }} \\
& =\left(\frac{V_{t d} V_{t b}^{*}}{V_{u d} V_{u b}^{*}}\right)\left(\frac{V_{u d} V_{u b}^{*}}{V_{t d} V_{t b}^{*}}\right)^{*} .
\end{aligned}
$$

The expression for $\operatorname{Im} \lambda$ can be written in terms of the unitarity angle $\alpha$ by first considering the following result from complex analysis. For a complex number $z=r e^{i \theta}$ where $\theta$ represents the angle to the real axis, the quantity $\operatorname{Im} \lambda$ can be written as [13]:

$$
\begin{aligned}
\operatorname{Im}\left(\frac{z}{z^{*}}\right) & =\operatorname{Im}\left(\frac{r e^{i \theta}}{r e^{-i \theta}}\right) \\
& =\operatorname{Im}\left(e^{+2 i \theta}\right) \\
& =\operatorname{Im}(\cos 2 \theta+i \sin 2 \theta) \\
& =\sin 2 \theta
\end{aligned}
$$

Therefore, using the relationship for $\sin 2 \alpha$ found in Equation 2.24:

$$
\operatorname{Im} \lambda=\operatorname{Im}\left\{\left(\frac{V_{t d} V_{t b}^{*}}{V_{u d} V_{u b}^{*}}\right)\left(\frac{V_{u d} V_{u b}^{*}}{V_{t d} V_{t b}^{*}}\right)^{*}\right\}=\sin 2 \alpha .
$$

Thus, the time-dependent asymmetry for the decay $B^{0} \rightarrow \pi^{+} \pi^{-}$provides information about the unitarity angle $\alpha$ in the following manner:

$$
a(t)_{B^{0} \rightarrow \pi^{+} \pi^{-}}=-\operatorname{Im} \lambda \sin (\Delta m t)=-\sin (2 \alpha) \sin (\Delta m t) .
$$

\subsubsection{The decay $B^{0} \rightarrow J / \psi K_{S}^{0}$}

The Feynman diagram for the decay $B^{0} \rightarrow J / \psi K_{S}^{0}$ given in Figure 2.9 shows that the amplitude, $\left\langle f_{C P} \mid B^{0}\right\rangle$ depends on the CKM matrix elements $V_{c b}^{*}$ and $V_{c s}$. 


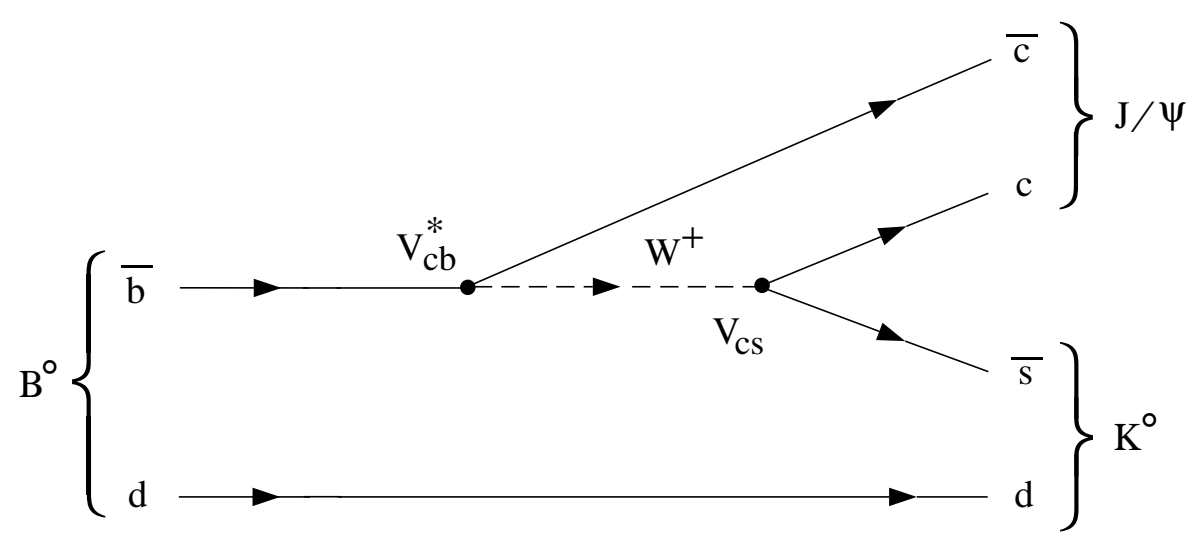

Figure 2.9: Feynman diagram for the decay $B^{0} \rightarrow J / \psi K_{S}^{0}$. The amplitude for this process will depend on the CKM matrix elements $V_{c b}$ and $V_{c s}$.

As in Section 2.5.1, $\lambda$ will depend on CKM matrix elements describing $B^{0}-\overline{B^{0}}$ mixing and $B$ decay; however, in this case, an extra factor will have to be included in $\lambda$ to account for the $K^{0}-\overline{K^{0}}$ mixing. Since mixing in the kaon system is expected to be dominated by the charm quark (see Figure 2.3), the additional $q / p$ factor will have the following form:

$$
\left(\frac{q}{p}\right)_{K \text { mixing }} \approx \sqrt{\frac{M_{12}^{*}}{M_{12}}}=\frac{V_{c d}^{*} V_{c s}}{V_{c d} V_{c s}^{*}} .
$$

Combining these contributions from the $B$ and $K$ mesons, $\lambda$ for the decay $B^{0} \rightarrow$ $J / \psi K_{S}^{0}$ is given as

$$
\begin{aligned}
\lambda_{B^{0} \rightarrow J / \psi K_{S}^{0}} & =\left(\frac{V_{t b}^{*} V_{t d}}{V_{t b} V_{t d}^{*}}\right)_{B \text { mixing }}\left(\frac{V_{c b} V_{c s}^{*}}{V_{c b}^{*} V_{c s}}\right)_{B \text { decay }}\left(\frac{V_{c d}^{*} V_{c s}}{V_{c d} V_{c s}^{*}}\right)_{K \text { mixing }} \\
& =\left(\frac{V_{t b} V_{t d}^{*}}{V_{c b} V_{c d}^{*}}\right)^{*}\left(\frac{V_{t b} V_{t d}^{*}}{V_{c d}^{*} V_{c b}}\right) .
\end{aligned}
$$

Using an expression similar to Equation 2.83:

$$
\begin{aligned}
\operatorname{Im}\left(\frac{z^{*}}{z}\right) & =\operatorname{Im}\left(\frac{r e^{-i \theta}}{r e^{i \theta}}\right) \\
& =\operatorname{Im}\left(e^{-2 i \theta}\right)
\end{aligned}
$$




$$
\begin{aligned}
& =\operatorname{Im}(\cos 2 \theta-i \sin 2 \theta) \\
& =-\sin 2 \theta,
\end{aligned}
$$

with the definition of $\beta$ in Equation 2.25, the expression for the imaginary part of $\lambda$ becomes:

$$
\operatorname{Im} \lambda=\operatorname{Im}\left\{\left(\frac{V_{t b} V_{t d}^{*}}{V_{c b} V_{c d}^{*}}\right)^{*}\left(\frac{V_{t b} V_{t d}^{*}}{V_{c d}^{*} V_{c b}}\right)\right\}=-\sin 2 \beta .
$$

Therefore, the time-dependent asymmetry for the decay $B^{0} \rightarrow J / \psi K_{S}^{0}$ is

$$
a(t)_{B^{0} \rightarrow J / \psi K_{S}^{0}}=-\operatorname{Im} \lambda \sin (\Delta m t)=+\sin (2 \beta) \sin (\Delta m t) .
$$

\subsubsection{The decay $B_{s}^{0} \rightarrow \rho K_{S}^{0}$}

The Feynman diagram for the decay $B_{s}^{0} \rightarrow \rho K_{S}^{0}$ given in Figure 2.10 shows that $\left\langle f_{C P} \mid B^{0}\right\rangle$ depends on the CKM matrix elements $V_{u b}^{*}$ and $V_{u d}$.

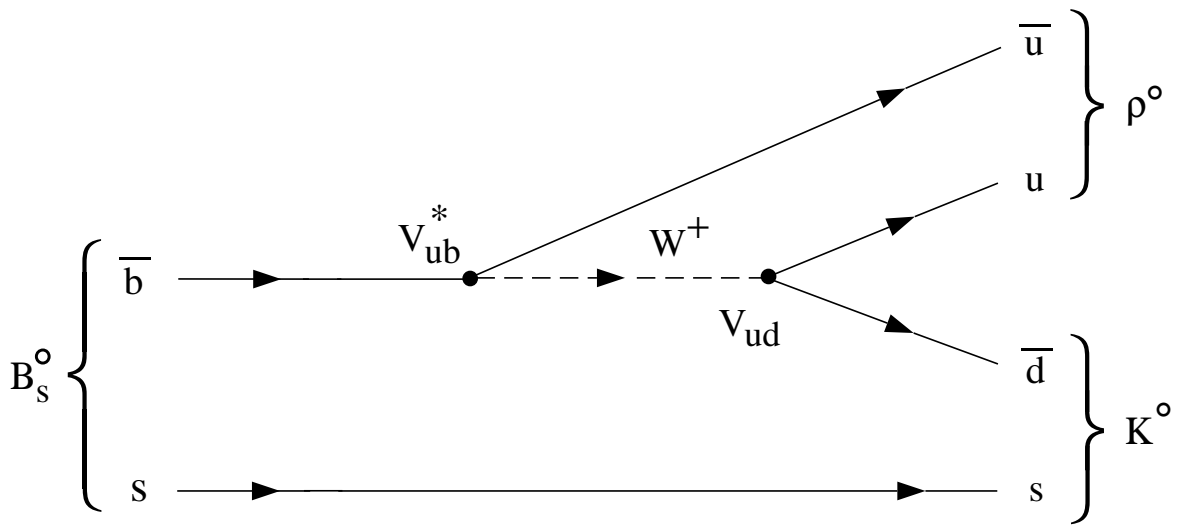

Figure 2.10: Feynman diagram for the decay $B_{s}^{0} \rightarrow \rho K_{S}^{0}$. The amplitude for this process will depend on the CKM matrix elements $V_{u b}$ and $V_{u d}$. Recall that in the Wolfenstein parameterization, $V_{u b}$ is expected to depend on the complex phase.

Once again, because of the $K$ mixing involved in producing the (approximate) $C P$ eigenstates, $\lambda$ will include the same additional contribution that was needed for the 
decay $B^{0} \rightarrow J / \psi K_{S}^{0}$ in Section 2.5.2. However, since this decay involves $B_{s}^{0}$, the $B$ mixing contribution to $\lambda$ will include $V_{t s}$ rather than $V_{t d}$ :

$$
\lambda_{B_{s}^{0} \rightarrow \rho K_{S}^{0}}=\left(\frac{V_{t b}^{*} V_{t s}}{V_{t b} V_{t s}^{*}}\right)_{B_{s} \text { mixing }}\left(\frac{V_{u b} V_{u d}^{*}}{V_{u b}^{*} V_{u d}}\right)_{B \text { decay }}\left(\frac{V_{c s}^{*} V_{c d}}{V_{c s} V_{c d}^{*}}\right)_{K \text { mixing }} .
$$

From the Wolfenstein parameterization in Equation 2.21, $\left|V_{t s}\right|$ has the parameterized form that $\left|V_{c b}\right|$ has, which means that the following approximation can be made:

$$
\frac{V_{t s}}{V_{t s}^{*}} \simeq \frac{V_{c b}}{V_{c b}^{*}}
$$

Moreover, $V_{t b}, V_{c d}, V_{c s}$, are all approximately real quantities, therefore the imaginary part of $\lambda$ can be written in terms of the unitarity angle $\gamma$ using Equation 2.87 and the definition of $\gamma$ in Equation 2.26 as follows:

$$
\operatorname{Im} \lambda=\operatorname{Im}\left\{\left(\frac{V_{u d} V_{u b}^{*}}{V_{c d} V_{c b}^{*}}\right)^{*}\left(\frac{V_{u d} V_{u b}^{*}}{V_{c d} V_{c b}^{*}}\right)\right\}=-\sin 2 \gamma .
$$

This in turn leads to a time-dependent asymmetry for the decay $B_{s}^{0} \rightarrow \rho K_{S}^{0}$ of the form:

$$
a(t)_{B_{s}^{0} \rightarrow \rho K_{S}^{0}}=-\operatorname{Im} \lambda \sin (\Delta m t)=+\sin (2 \gamma) \sin (\Delta m t)
$$

\subsubsection{Summary}

Table 2.2 is a summary of the most relevant information for each of the proposed decays, including the branching ratio and the unitarity angle that it is being used to study. 


\begin{tabular}{|l|c|c|c|}
\hline Decay & Quark Process & Branching Ratio & Unitarity Angle \\
\hline \hline$B^{0} \rightarrow \pi^{+} \pi^{-}$ & $b \rightarrow u \bar{u} d$ & $<2.9 \times 10^{-5}($ at $90 \%$ C.L. $)$ & $\sin 2 \alpha$ \\
$B^{0} \rightarrow J / \psi K_{S}^{0}$ & $b \rightarrow c \bar{c} s$ & $(7.5 \pm 2.1) \times 10^{-4}$ & $-\sin 2 \beta$ \\
$B_{s}^{0} \rightarrow \rho K_{S}^{0}$ & $b \rightarrow u \bar{u} d$ & $<3.2 \times 10^{-4}($ at $90 \%$ C.L. $)$ & $-\sin 2 \gamma$ \\
\hline
\end{tabular}

Table 2.2: Table indicating the relevant decay information and $C P$ physics for each of the proposed decays. The branching ratios for the respective decays were obtained from the values published by the Particle Data Group [2]. 


\section{Chapter 3}

\section{The $B A B A R$ Detector}

\subsection{Introduction}

This chapter will serve to give an overview of the $B A B A R$ detector that will be used at the SLAC B Factory, highlighting some of the main motivations for the present design. As outlined in Chapter 2, the study of $C P$ violation in the $B$ meson system involves measuring the asymmetries in the decays of $B^{0}$ and $\overline{B^{0}}$ mesons to $C P$ eigenstates. The physics requirements in such an experiment include the ability to produce $B$ mesons, the ability to reconstruct $B$ meson decays to $C P$ eigenstates, and the ability to measure the relative decay times of the two $B$ mesons. As will be outlined below in more detail, the first of these requirements may be fulfilled at an $e^{+} e^{-}$collider, the second requirement by a high luminosity beam facility, and the third by employing asymmetric beam energies.

\section{2 $\quad B$ Meson Production}

\subsubsection{PEP-II Storage Ring}

The beam facility at SLAC that will be used in the B Factory experiment is PEP-II, and is illustrated in Figure 3.11. PEP-II is an asymmetric $e^{+} e^{-}$storage ring in which $9 \mathrm{GeV}$ electrons collide with $3.1 \mathrm{GeV}$ positrons to annihilate. The initial energy from this collision then goes into creating other final state particles due to the equivalence 
of mass and energy stated in the equation, $E=m c^{2}$.

Electrons (denoted by dark particles in Figure 3.11) produced in the electron gun first pass through a damping ring, the purpose of which is to narrow the energy spectrum so that the energies of the electrons are better defined. The electrons are then accelerated along a 2 mile long injector line. Along the way, some of the electrons are directed away from the main beamline and are used to produce positrons (denoted by white particles in the diagram) by allowing the electrons to strike a metal target in the region labeled "positron source". Once the positrons are created, they travel back along the positron return line so that they can enter the other damping ring. Just as the positrons are emerging from their damping ring, a corresponding bunch of electrons is doing the same, and together, in the same beamline, they accelerate toward the storage rings at the end of the injector line. A particle detector is placed at the point where the electron beam and positron beam collide.

Instead of colliding electrons and positrons one at a time, it is much easier to direct bunches of electrons at bunches of positrons to increase the chance of a successful collision. At SLAC, electrons and positrons are produced in bunches of $3 \times 10^{10}$ and $6 \times 10^{10}$ respectively, and each bunch is separated by approximately $1 \mathrm{~m}$ [14].

Even using bunches of particles, it is often very difficult to create conditions in which an electrons from one direction will collide with positrons coming in the other direction. However, using a ring facility means that if at first the two bunches of particles do not annihilate, they can be recycled for another collision attempt. Also, the magnets used to focus the two particle beams are located very near to the interaction region, allowing for very accurate positioning. The beam size at the interaction point is $6.2 \mu \mathrm{m} \times 155$ $\mu \mathrm{m}[14]$.

Given sufficient energy, the colliding electron and positron beams may produce a resonance particle, the $\Upsilon(4 \mathrm{~S})$, which has a mass of $10.58 \mathrm{GeV} / \mathrm{c}^{2}[2]$. The $\Upsilon(4 \mathrm{~S})$ will in 


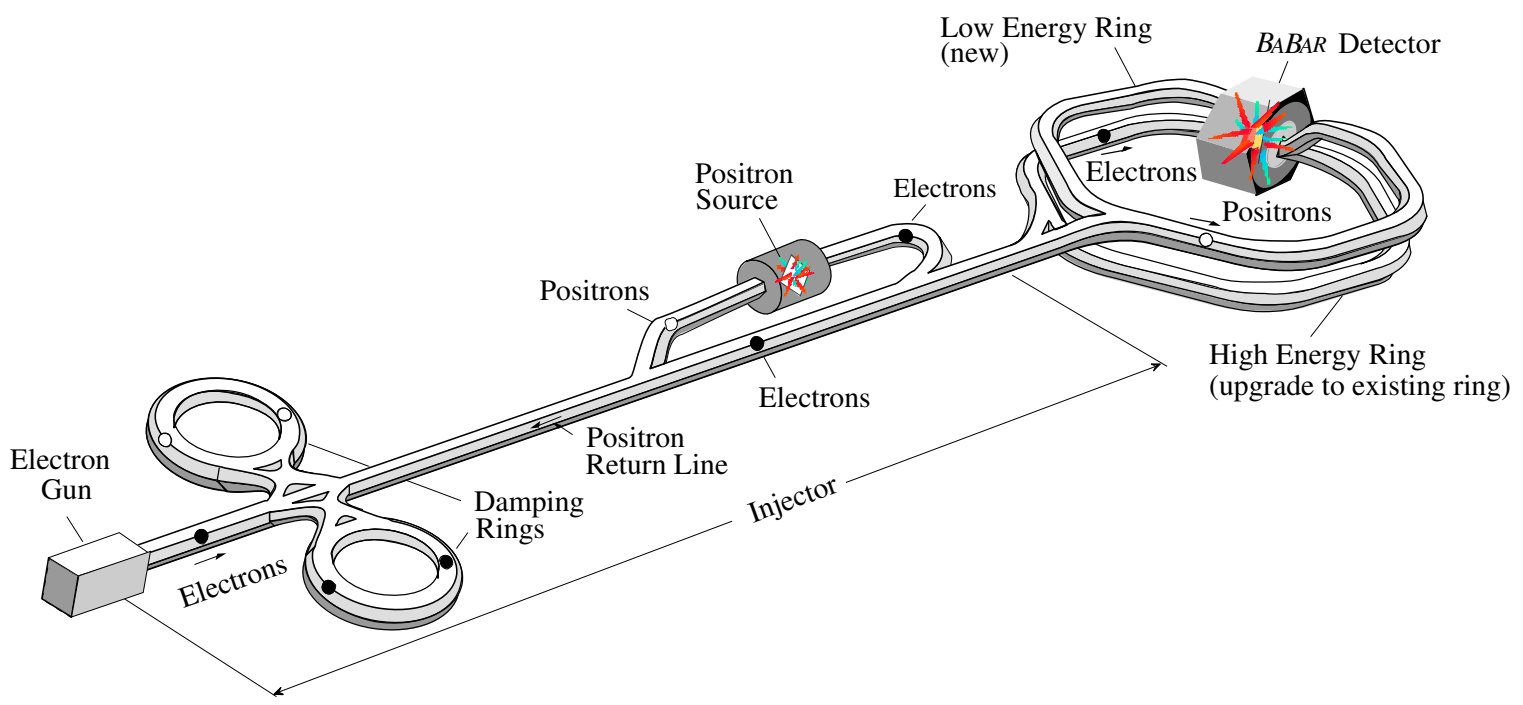

Figure 3.11: Shown is a picture of the PEP-II storage ring facility at SLAC. Electrons and positrons are accelerated along the injector line, enter the storage rings, and collide in the particle detector.

turn decay approximately half of the time to two neutral $B$ mesons ${ }^{8}$, each having a mass of $5.279 \mathrm{GeV} / \mathrm{c}^{2}[2]$ :

$$
e^{+} e^{-} \rightarrow \Upsilon(4 \mathrm{~S}) \rightarrow B^{0} \overline{B^{0}} .
$$

Since the mass of the $\Upsilon(4 \mathrm{~S})$ is only marginally greater than the combined mass of two $B$ mesons, the $B^{0}$ and $\overline{B^{0}}$ produced in the decay will have negligible momentum in the centre of mass frame.

\subsection{2 $B^{0}-\overline{B^{0}}$ Orthogonality}

The $\Upsilon(4 \mathrm{~S})$ state that is produced in the annihilation of energetic electrons and positrons is a resonance state where the $(4 \mathrm{~S})$ label " $\mathrm{S}$ " signifies that the resonance is in a state of orbital angular momentum $L=0$. The total angular momentum of the $\Upsilon(4 \mathrm{~S})$ is

\footnotetext{
${ }^{8}$ This treatment is for $B^{0}$ mesons and not $B_{s}^{0}$ mesons because the $\Upsilon(4 \mathrm{~S})$ does not have enough mass to decay into a $B_{s}^{0} \overline{B_{s}^{0}}$ pair. In order to produce $B_{s}^{0}$ mesons after the initial $B^{0}$ data have been taken, PEP-II may operate at the $\Upsilon(5 \mathrm{~S})$ resonance which has the necessary mass.
} 
known to be $J=1$ where $J$ is defined as

$$
J=L+S
$$

which implies that the spin angular momentum, $S=1$. Conservation of total angular momentum dictates that the total angular momentum of the system before a reaction must equal the total final angular momentum of the system; however, the orbital and spin components of the total angular momentum are not separately conserved before and after the reaction. In the case of of the decay $\Upsilon(4 \mathrm{~S}) \rightarrow B^{0}+\overline{B^{0}}$, the statement of angular momentum conservation is given as follows:

$$
J_{\Upsilon(4 \mathrm{~S})}=1=J_{B^{0}}+J_{\overline{B^{0}}} .
$$

Because the $B^{0}$ and $\overline{B^{0}}$ mesons are spin-0 particles $(S=0)$, the relative orbital angular momenta of the two $B$ mesons is constrained to be unity in order to maintain $J=1$ in Equation 3.96:

$$
L=L_{B^{0}}+L_{\overline{B^{0}}}=1 .
$$

$B$ mesons have integer spin which means that they are classified as bosons. According to Bose statistics, the total wavefunction for a system of identical bosons must be symmetric under the interchange of the particles, which means that $L+S \equiv$ even. However, if a system of two identical particles is in a combined state of orbital angular momentum $L=1$, and have a combined spin angular momentum of $S=0$, that implies that $L+S \equiv$ odd, which is a characteristic of an antisymmetric wavefunction. Initially, when the $B^{0} \overline{B^{0}}$ pair is produced, the $B$ mesons are in orthogonal states, one state is a $B^{0}$ meson and the other is a $\overline{B^{0}}$. From the angular momentum considerations above, the $B^{0}$ and $\overline{B^{0}}$ can never mix so as to form the same identical particle, because this would result in a symmetric wavefunction which does not satisfy $L+S=1+0 \equiv$ odd. For instance, a $B^{0}$ can change into a $\overline{B^{0}}$ through mixing, but it cannot do so at the 
same time that another $\overline{B^{0}}$ exists due to the above angular momentum constraints. Having two different particles in a state of $L+S \equiv$ odd is not forbidden by any angular momentum argument. Therefore, as time evolves, Bose statistics forces the orthogonality condition to exist throughout the mixing process until one of the $B$ mesons finally does decay, as shown in Figure 3.12. If one of the $B$ mesons is observed to decay to a $B^{0}$ state at time $t$, then at that instant it is known to be in a pure $B^{0}$ state and, as a consequence, at the same instant there was a corresponding pure $\overline{B^{0}}$ state due to the orthogonality condition. The point in time of this known decay effectively starts the clock for the remaining particle $\left(B^{0}\right.$ or $\left.\overline{B^{0}}\right)$ to evolve due to mixing with its antiparticle. The time of the decay to a $C P$ eigenstate, $t_{C P}$, is expressed relative to the time at which one of the $B$ mesons decays to a state, $t_{t a g}$, which "tags" the bottom quark flavour of the meson as either $b$ or $\bar{b}$ :

$$
t \equiv t_{C P}-t_{t a g}
$$

Depending on which $B$ meson is used to tag the event, the time, $t$, can be either positive or negative. Semileptonic decays of the form $B^{0} \rightarrow \ell^{+} X$ or $\overline{B^{0}} \rightarrow \ell^{-} X$ are often used to tag the quark flavour ${ }^{9}$ of the parent meson. Therefore, one requirement of the $B_{A} B_{A R}$ detector will be that it must have the ability to reconstruct the decay to the $C P$ eigenstate as well that of the tagging state which tags the quark content of one of the $B$ mesons at the instant of the decay time.

\subsection{Reconstruction of $B$ Decay Vertices}

The fact that the electron and positron energies at PEP-II are asymmetric addresses the need to reconstruct the $B$ decay vertices. Recall from Equation 2.79 that the time

\footnotetext{
${ }^{9}$ The term flavour tagging simply refers to determining whether the quark was a $b$ or $\bar{b}$. Recall from Chapter 2 that quarks exist in three families, each quark having a corresponding antiquark; flavour is a general term denoting a particular family.
} 


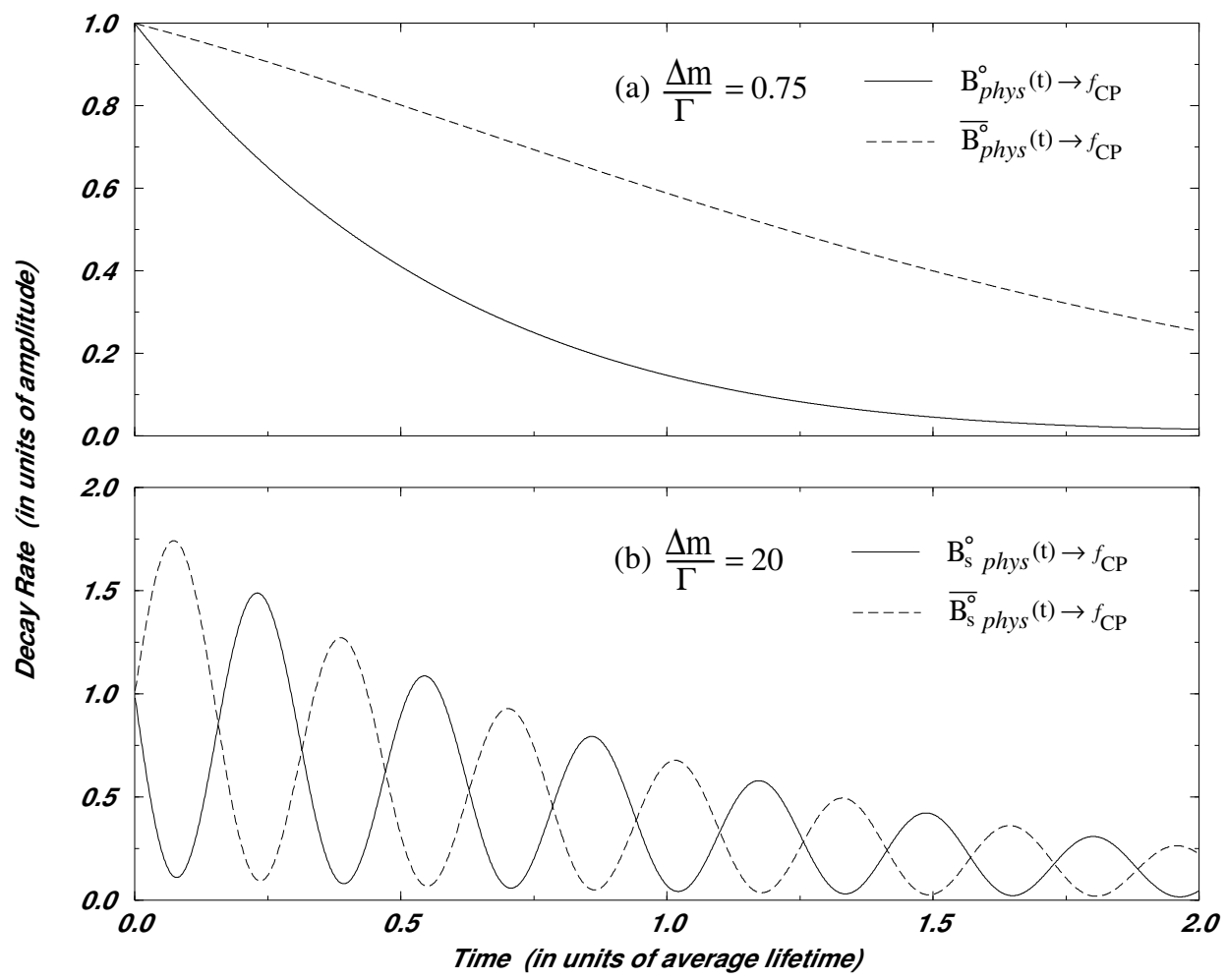

Figure 3.12: Shown is the orthogonality between the $B^{0}$ and $\overline{B^{0}}$ decay for $\operatorname{Im} \lambda=$ 0.88 and two mixing rates, $\Delta m / \Gamma$. Plot (a) shows the superposition of exponential decay and the orthogonal mixing condition for a mixing rate $\Delta m / \Gamma=0.75$ which is the rate expected for $B^{0}-\overline{B^{0}}$ mixing [11]. Plot (b) shows a similar curve for a mixing rate $\Delta m / \Gamma=20$ which is the mixing rate expected in the $B_{s}^{0}$ system [11].

difference in the lifetimes of the $B^{0}$ and the $\overline{B^{0}}$ decays is essential to the understanding of the $C P$ violation. The easiest way to measure such a time difference is to determine to the spatial distance between the two decays and, knowing the velocities of the particles, calculate the time difference.

Consider two beams of equal energy first. Since the $\Upsilon(4 \mathrm{~S})$ is produced at rest in the laboratory and is only slightly above the mass threshold for the decay to two $B$ mesons, the $B^{0}$ and the $\overline{B^{0}}$ will decay at essentially the same point in space as illustrated in Figure 3.13(a). The difference between the respective decay times will be very difficult 
to measure because the distance from one decay vertex to the other will be very small. However, if one of the two colliding beams has a higher energy than the other, then, in the laboratory frame, the $\Upsilon(4 \mathrm{~S})$ produced in the $e^{+} e^{-}$collision would be boosted, thereby leaving a large distance between the two decays so that it can be measured with greater accuracy. The strategy at PEP-II [15] is to have a $9 \mathrm{GeV}$ electron beam collide with a $3.1 \mathrm{GeV}$ positron beam, resulting in a net boost, $\beta$, of approximately $0.5(v=0.5 \mathrm{c})$. Due to the unequal energies, when the $\Upsilon(4 \mathrm{~S})$ decays to a $B^{0} \overline{B^{0}}$ pair in the laboratory frame, the $B$ mesons will also be moving along the direction of the higher energy electron beam, as shown in Figure 3.13(b). The separation distance, $\Delta z$, is related to the time difference between the two $B$ decays through the expression:

$$
t=\frac{\Delta z}{\beta \gamma c}
$$

where $\gamma$ is related to $\beta$ in the following way:

$$
\gamma=\sqrt{\frac{1}{1-\beta^{2}}}
$$

Thus, measuring the spatial separation of the two $B$ meson decay vertices gives information about the decay time difference of the $B^{0}$ and $\overline{B^{0}}$ mesons. This time difference is then used to calculate the time-dependent $C P$ asymmetry that was given in Chapter 2 as:

$$
a_{f_{C P}}(t)=-\operatorname{Im} \lambda \sin (\Delta m t)
$$

\subsection{High Luminosity}

Recall from Table 2.2 that the branching ratios for $B$ mesons to $C P$ eigenstates are typically very small, on the order of $10^{-5}$. Therefore, in order to detect a sufficient number of $B$ decay events, the luminosity of the beam facility must be high enough 


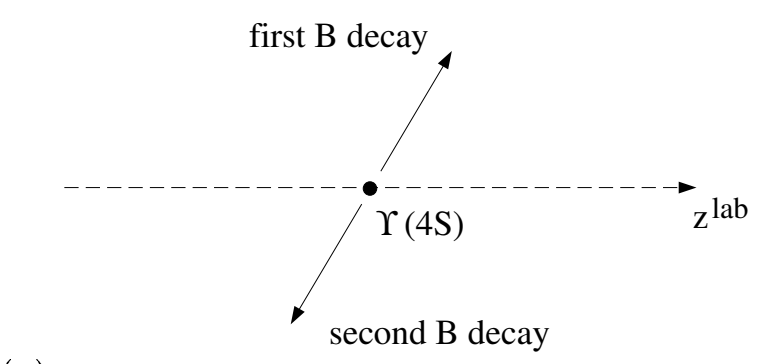

(a)

Figure 3.13: Diagram showing the effects of having (a) equal beam energies, and (b) unequal beam energies when trying to separate decay vertices. In the case where the beam energies are equal, there is essentially no spatial separation of the decay vertices: $\Delta z \approx 0$. While the two $B$ mesons may decay at different times, spatially both mesons decay at approximately the point where the $\Upsilon(4 \mathrm{~S})$ is created. Using beams of unequal energies, however, leads to a very pronounced spatial separation of the decay vertices: $\Delta z \approx 250 \mu \mathrm{m}$ in the case where $9 \mathrm{GeV}$ electrons are colliding with $3.1 \mathrm{GeV}$ positron beams.

to compensate for the low probabilities of decay. The luminosity of a particle beam is defined to be the number of particles incident on a certain area in a given time interval. Having more $B$ particles to decay offsets the fact that the $C P$ eigenstate decays are themselves rare. It is for this reason that the PEP-II group at SLAC is aiming to have an unprecedented peak luminosity of $3 \times 10^{33} \mathrm{~cm}^{-2} \mathrm{~s}^{-1}$, with a possible upgrade to $1 \times 10^{34} \mathrm{~cm}^{-2} \mathrm{~s}^{-1}$. Two key ingredients will contribute to this high peak luminosity. First, electron bunches will be sent every 4.2 ns, contributing to a very high number of possible reactions. Second, the beam focusing magnets will be positioned much closer than usual to the interaction region to allow for very accurate positioning. The latter gives rise to concerns about background rates caused when particles collide with the magnets, and is currently being studied. 


\subsection{Multiple Scattering}

In order to measure momentum and position very well, even in the more exterior detectors, such as the particle identification system and the electromagnetic calorimeter used for measuring energy, the mass of the tracking chambers must be kept as low as possible to reduce multiple scattering. This requirement applies in particular to the silicon vertex detector and the drift chamber components which lie closest to the interaction point and could potentially scatter the decay particles. For measuring energies in the electromagnetic calorimeter, the mass of the particle identification modules also becomes important.

\subsection{Overview of the $B A B A R$ Detector}

The $B_{A} B_{A R}$ detector illustrated in Figure 3.14 is composed of several sub-detectors each of which is responsible for fulfilling one or more of the physics requirements outlined above. The main components of the detector include a vertex detector, a central drift chamber, a system for particle identification, an electromagnetic calorimeter, and a muon and neutral hadron detector.

\subsubsection{Vertex Detector}

The main task of the vertex detector is to reconstruct the two primary decay vertices by measuring track angles in order to determine the time interval between the two $B$ meson decays. To do so, it must be able to measure distances on the order of $90 \mu \mathrm{m}[14]$; however, a more precise vertex detector will improve the background rejection and vertex-finding efficiency. A second requirement of the vertex detector is that it must also provide full tracking information for particles close to the interaction point with low transverse momenta $\left(p_{t}<100 \mathrm{MeV} / \mathrm{c}\right)$ that will not have sufficient energy to enter 


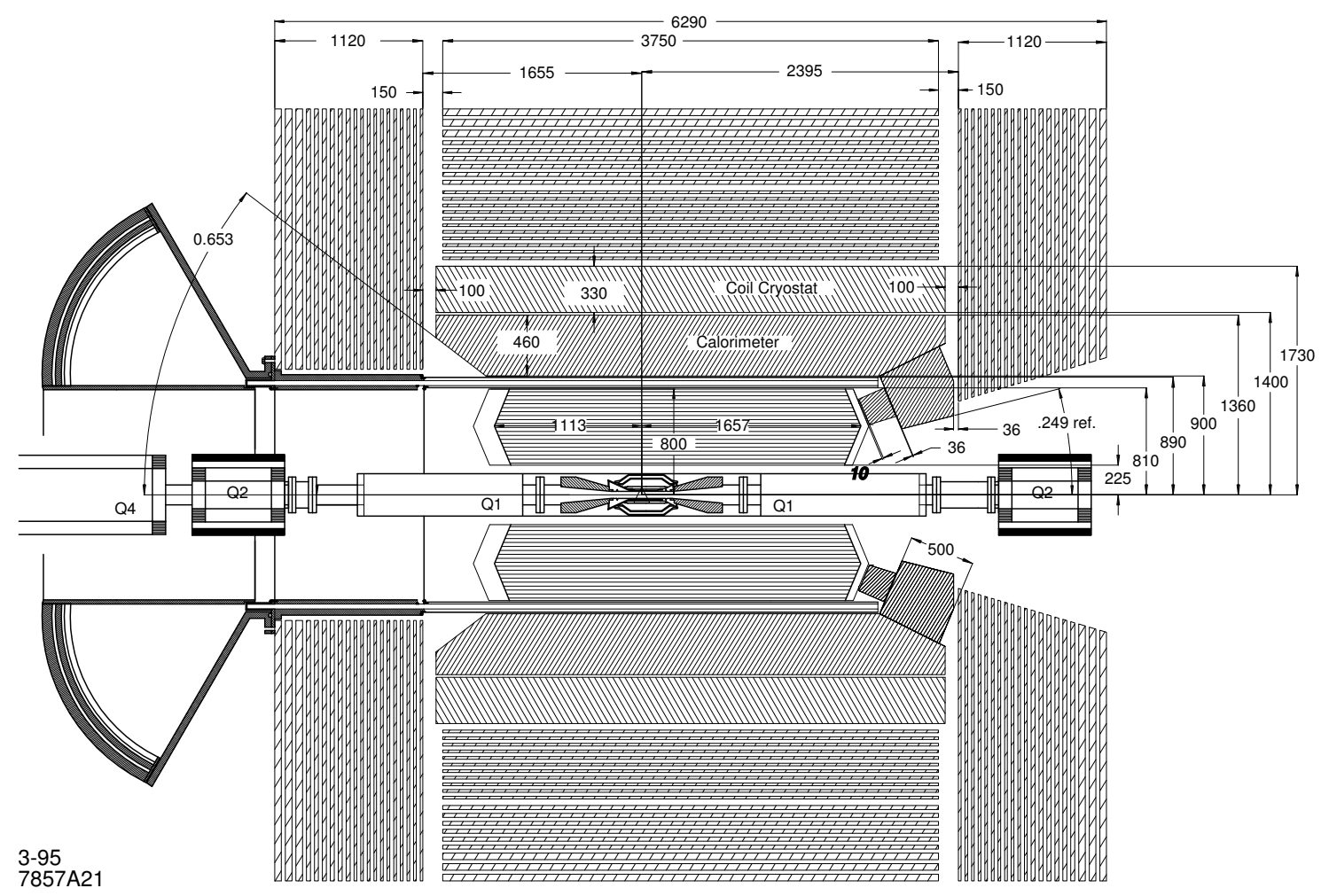

Figure 3.14: Shown is a picture of the entire $B_{A} B_{A R}$ detector as it was envisioned at the time of the Technical Design Report [14]. The dimensions given are all in millimeters. Starting at the interior and moving outward, the detector components shown are the silicon vertex tracker, the drift chamber, the particle identification system, the electromagnetic calorimeter, and the instrumented flux return housing the muon and hadron detector. Some of the beamline magnets (namely, Q1, Q2 and Q4) near the interaction region are shown as they aid in producing the high luminosity beam required at PEP-II. 
the drift chamber, which is normally where momentum is measured. A track is best measured using a number of detecting layers. Therefore, the vertex detector must have more than one layer so that it can trace out the trajectory of a particle. Because of this ability to provide track information, the $B_{A} B_{A R}$ vertex detector is known as the silicon vertex tracker (SVT).

The SVT illustrated in Figure 3.15 is constructed with 5 layers of double-sided silicon microstrip detectors, and occupies the radial space between $2.5 \mathrm{~cm}$ and $22 \mathrm{~cm}$ from the interaction point. High precision measurements of spatial distances are obtained using finely spaced silicon strips, on the order of $50 \mu \mathrm{m}$ for the inner three layers and between 65-100 $\mu \mathrm{m}$ for the outer three layers.

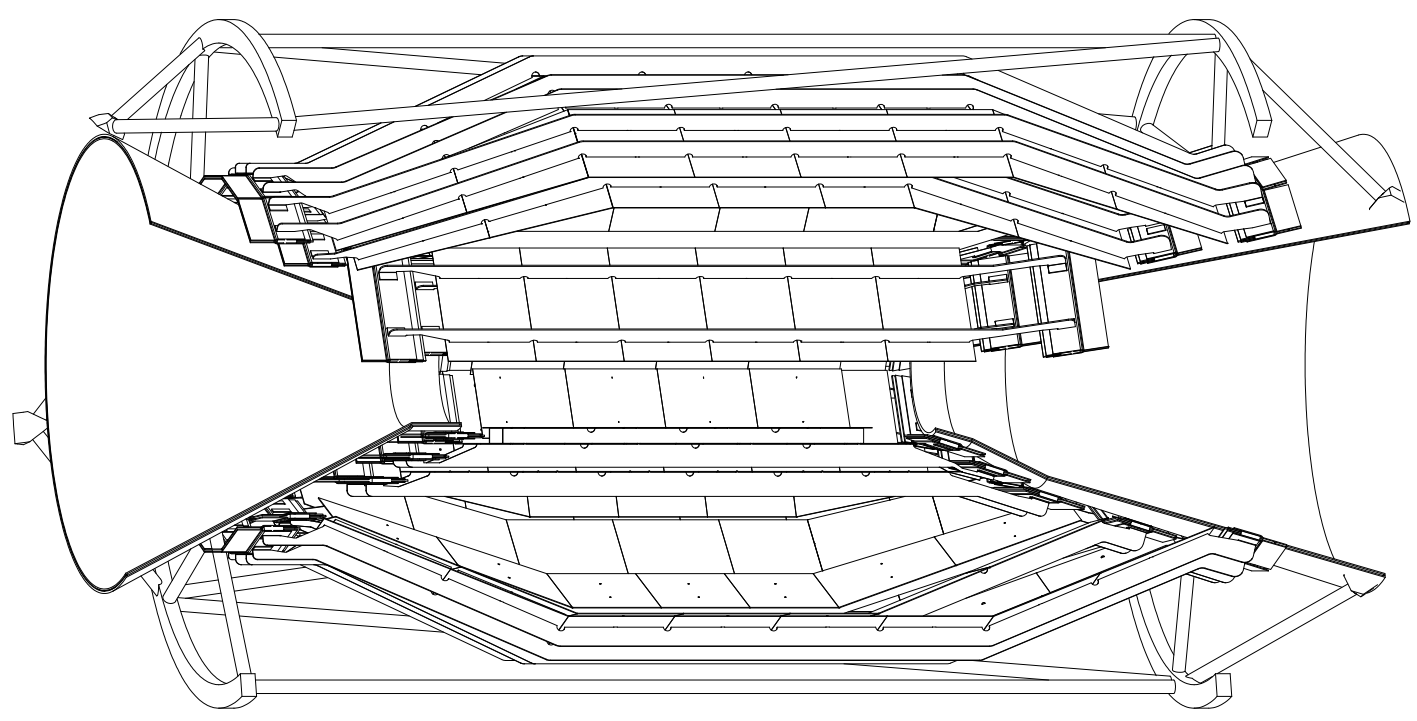

Figure 3.15: Diagram showing the silicon vertex tracker (SVT) [14]. The 5 layers are used to provide tracking information for particles which will not have sufficient energy to enter the drift chamber. 


\subsubsection{Drift Chamber}

The main purpose of the drift chamber is to provide good knowledge of charged particle momenta, as well as a good $d E / d x$ particle identification measurement for low momentum particles which do not reach the dedicated particle identification system, and backward going particles for which there is no particle identification. Since studies related to drift chamber performance are the main focus of this thesis, an entire chapter, Chapter 4, will be devoted to the discussion of its principles. However, a quick overview is in order.

At the time of the Technical Design Report, the $B_{A} B_{A R}$ drift chamber was proposed to be a large cylindrical container $280 \mathrm{~cm}$ in length with an inner radius of $22.5 \mathrm{~cm}$ and an outer radius of $80 \mathrm{~cm}$ as shown in Figure 3.16. Notice from the schematic that the interaction point (IP) is not located in the center of the chamber, but rather is offset to the left. The drift chamber is designed this way in order to take advantage of the asymmetric beam energies. Most of the particles produced in the $e^{+} e^{-}$collisions will be moving in the forward direction, which in this case is to the right. An asymmetric drift chamber such as the one proposed for the SLAC B Factory will therefore have increased sensitivity in the region where most of the particles will be. The chamber itself will be strung with approximately 7000 gold plated tungsten sense wires $20 \mu \mathrm{m}$ in diameter, 45000 gold plated aluminum field wires $55 \mu \mathrm{m}$ in diameter, filled with gas and immersed in a $1.5 \mathrm{~T}$ magnetic field. The trajectory of the particle is measured as it traverses a maximum of 40 layers of sense wires in the chamber, ionizing chamber gas in each. The points of ionization are connected to form a curved path from which the charge and momentum of the particle can be inferred, in conjunction with the specific ionization deposition $(d E / d x)$, which becomes important in identifying particle species.

To minimize multiple scattering, helium-based gases are being tested to determine 


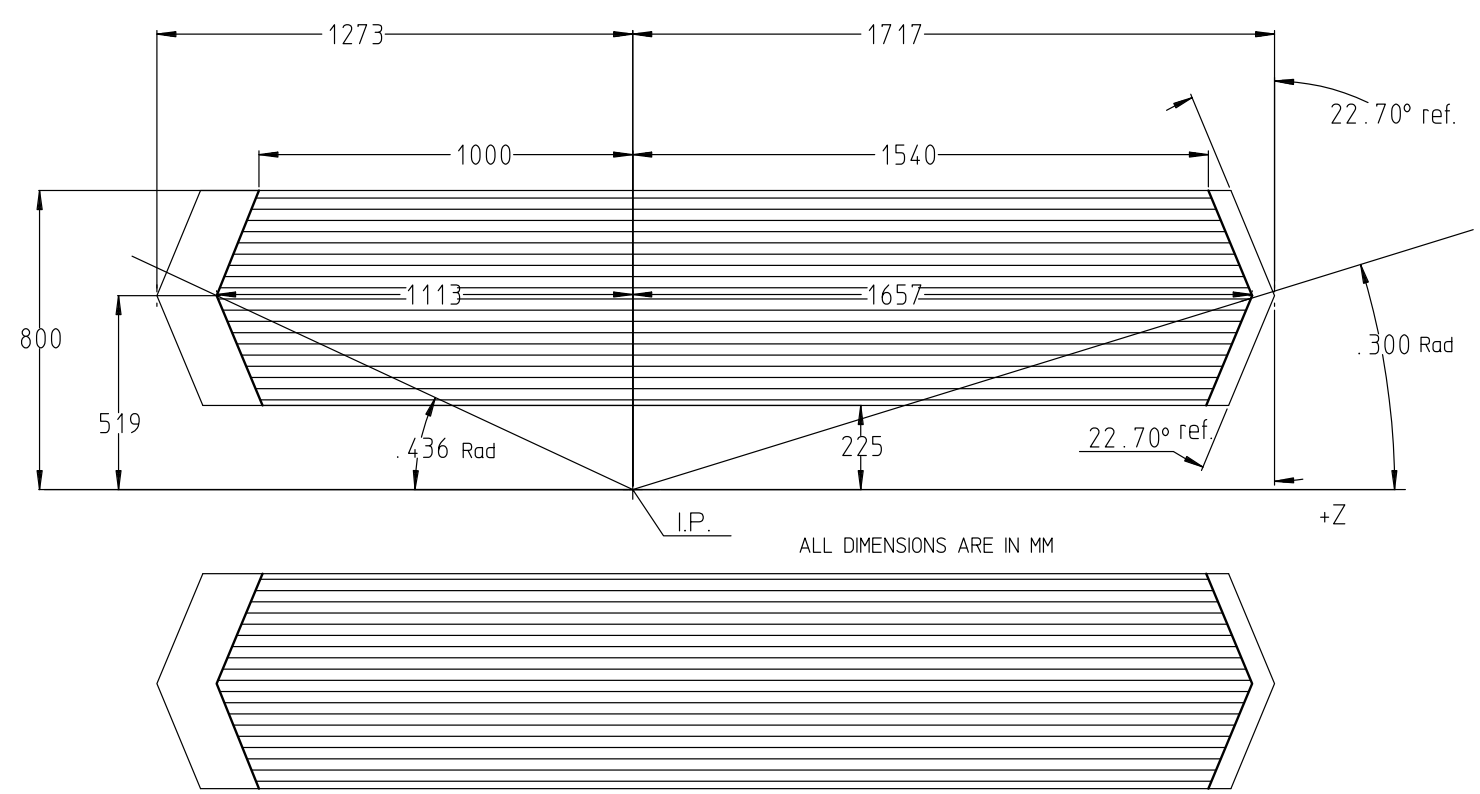

Figure 3.16: Diagram showing the drift chamber [14]. The view depicts a side view of the chamber with the sides cut away - the real chamber will form a complete cylinder. The interaction point (IP) is not located in the center of the chamber, but rather is offset to the left in order to take advantage of the asymmetric beam energies.

whether or not they can perform at a level comparable to the more traditional argonbased gases that have been used in the past. Aluminum field wires will also be used in the chamber to reduce mass.

\subsubsection{Particle Identification}

Knowing which particles came from which event is very important in any particle physics experiment. In the case of the $B_{A} B_{A R}$ detector, information from the drift chamber, electromagnetic calorimeter and instrumented flux return can be used to identify most of the leptons and many species of hadrons. However, an additional dedicated particle identification system is needed to distinguish charged pions from 
charged kaons and at higher momenta, distinguish charged pions from protons. In order to meet this requirement, a combination of Čerenkov detectors will be incorporated into the $B_{A} B_{A R}$ detector. The barrel region will be covered by a DIRC (Detection of Internally Reflected Čerenkov light) system, while an ATC (Aerogel Threshold Čerenkov counter) covers the forward region as shown in Figure $3.17^{10}$

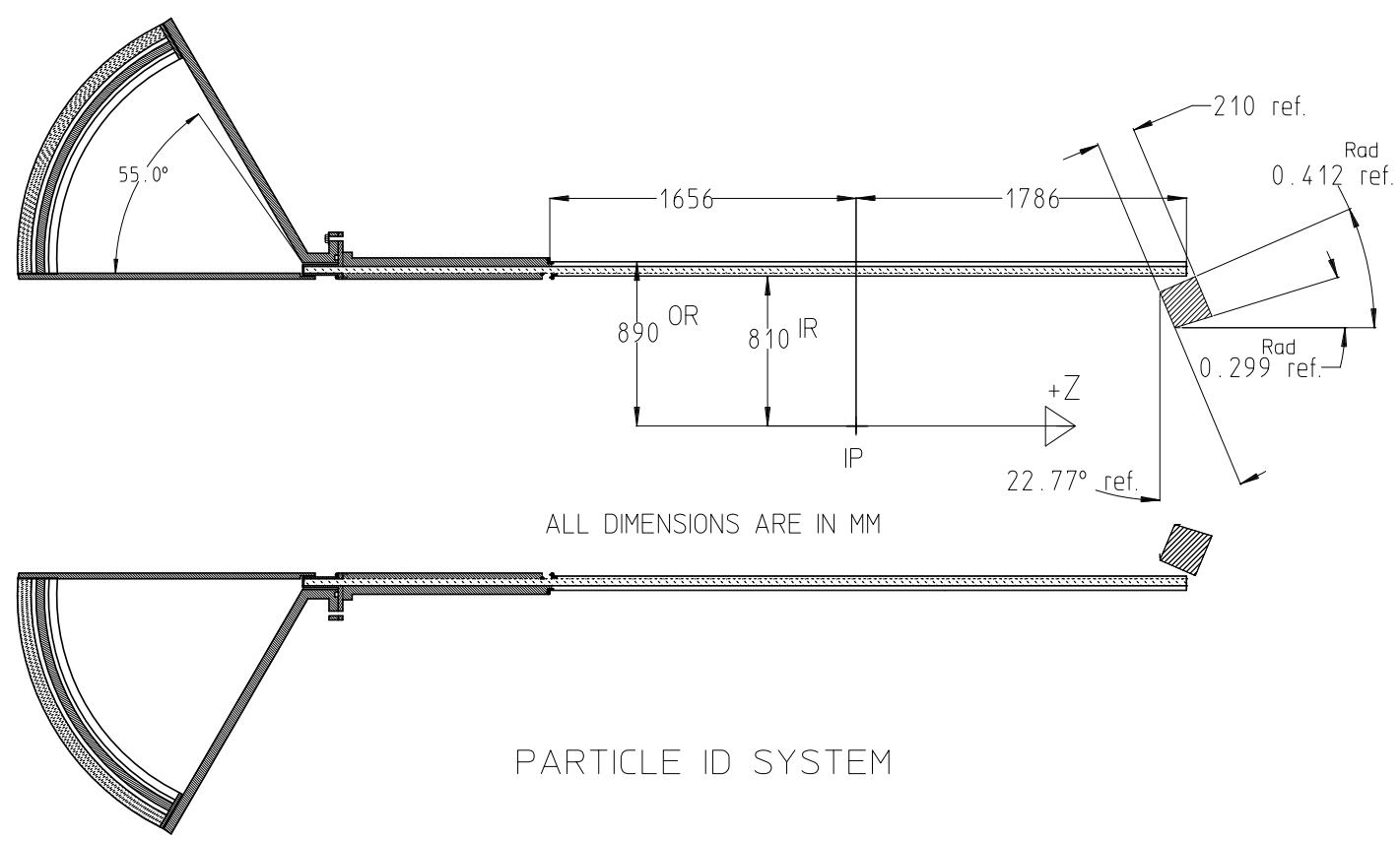

Figure 3.17: Shown is a side view of the particle identification system [14]. The DIRC comprises 156 thin quartz sectors positioned cylindrically in a 12-sided polygon parallel to the beampipe. The wedge shaped objects at the far left of the diagram house the mirrors used to direct the light from the quartz bars to the photomultiplier tubes as well as offer a support structure for the photomultiplier tubes themselves. The two small squares at the right end of the detector indicate the position of the ATCs.

When a charged particle in a certain medium travels faster than the speed of light in that same medium, an electromagnetic shock wave is formed that is conical in shape about the trajectory of the particle, as shown in Figure 3.18. The shock wave radiation

\footnotetext{
${ }^{10}$ Aerogel counters have recently been phased out of the $B A B A R$ detector design, but a brief mention is included here because at the time of the Technical Design Report [14] they were present.
} 
is called $\check{C}$ erenkov radiation, and the angle at which it is emitted depends on the particle velocity and the refractive index of the medium. Therefore, measuring the angle of the Cerenkov light cone determines the velocity of the particle. If the momentum of the particle in question is known using drift chamber measurements, and the velocity is known using the Čerenkov counters, then the mass of the particle is known, which can be used to identify the particle species. However, due to the finite resolution of the detector, it is often very difficult to separate pions from muons based on their mass alone. Unlike the pion and kaon for instance, the mass difference between the pion and muon is far too small for the particle identification system to distinguish between the two, which is the reason that an additional muon detector is required.

The DIRC detector shown in Figure 3.19 is an internal reflection imaging device that uses 156 thin quartz bars arranged in a 12-sided polygon around the beam line to generate and transport a Čerenkov cone to a huge water-filled tank. Within the water, the Čerenkov cone image is allowed to expand in diameter and is then detected by an array of approximately 13400 photomultiplier tubes located on the exterior of the tank. The reason water is used is because it transports ultraviolet Čerenkov light well and is very inexpensive.

The ATC system consists of 144 blocks of aerogel arranged in two C-shaped sections having two indices of refraction that are chosen to provide optimal separation of kaons and pions over the full momentum range.

\subsubsection{Electromagnetic Calorimeter}

The most expensive component of the $B_{A} B_{A R}$ detector will be the electromagnetic calorimeter which will measure photon energy using an array of approximately 6000 thalium doped cesium iodide crystals, $\mathrm{CsI}(\mathrm{Tl})$. Measuring photon energy is extremely important at the B Factory because photons constitute the final product in several of 


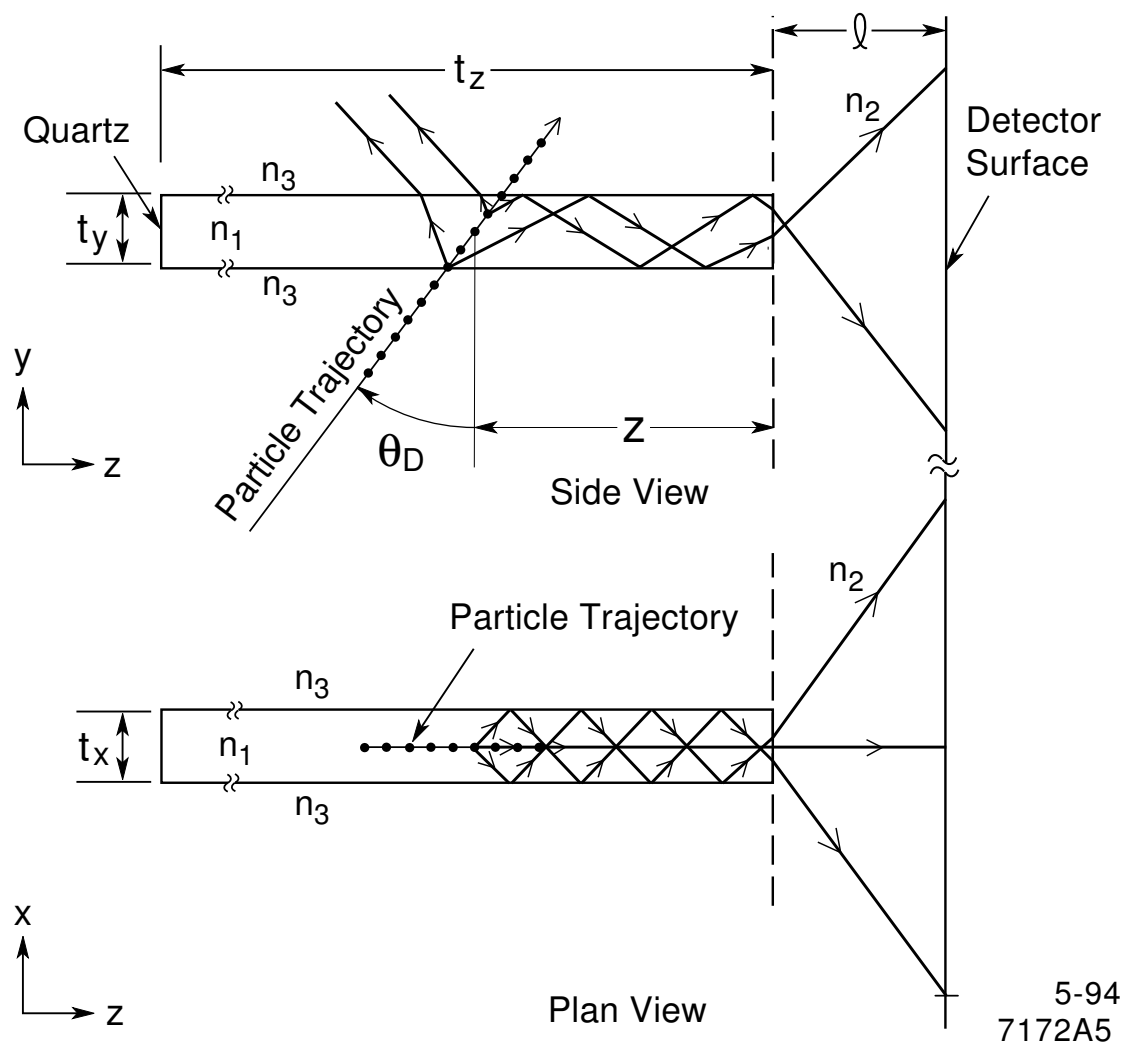

Figure 3.18: Shown is an schematic of a single quartz bar in the DIRC detector illustrating the principle behind Čerenkov counters [14]. As a particle enters the quartz bar, a Cerenkov cone is created which then propagates to the end of the bar and onto a detector surface.

the key $C P$ violation decays, including the decay $B^{0} \rightarrow J / \psi K_{S}^{0}$ and $B_{s}^{0} \rightarrow \rho K_{S}^{0}$.

For example, the decay $B^{0} \rightarrow J / \psi K_{S}^{0}$ yields two neutral pions approximately one third of the time through the subsequent decay of the $K_{S}^{0}$ :

$$
K_{S}^{0} \rightarrow \pi^{0} \pi^{0}
$$

The $\rho$ meson in the decay $B^{0} \rightarrow \rho^{+} \pi^{-}$also contributes a neutral pion through the decay mode: $\rho^{+} \rightarrow \pi^{+} \pi^{0}$. Because neutral pions have a signature decay to two photons 


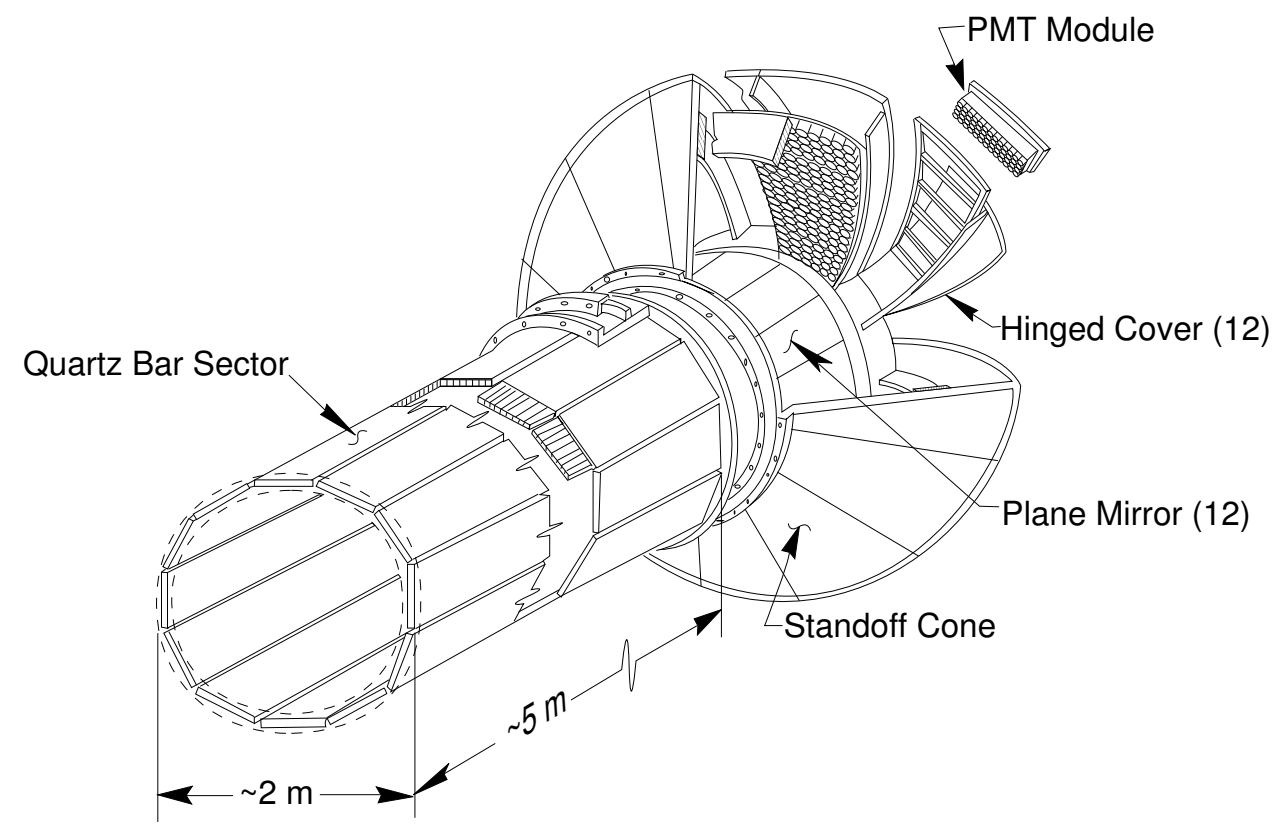

Figure 3.19: Shown is an illustration of the DIRC [14] particle identification system. The DIRC comprises 156 thin quartz sectors positioned in a 12-sided cylindrical polygon parallel to the beampipe. The wedge shaped objects at the top of the diagram house the mirrors and water used to direct the light from the quartz bars to the photomultiplier tubes, as well as offer a support structure for the photomultiplier tubes.

$98.8 \%[2]$ of the time,

$$
\pi^{0} \rightarrow \gamma \gamma
$$

an excellent photon detector is essential in order to measure the presence of $\pi^{0}$ mesons that are produced in various decay chains.

Shown in Figure 3.20 is a side view of the calorimeter barrel and forward endcap; the full detector is cylindrically symmetric about the beam axis. Notice that the electromagnetic calorimeter does not extend completely in the backward direction to cover the rear endcap. Due to the asymmetric beam energies that produce a boost in the forward direction, few particles will have momentum in the backward direction, 
and due to the high cost involved, it was felt that the present design was the best compromise.

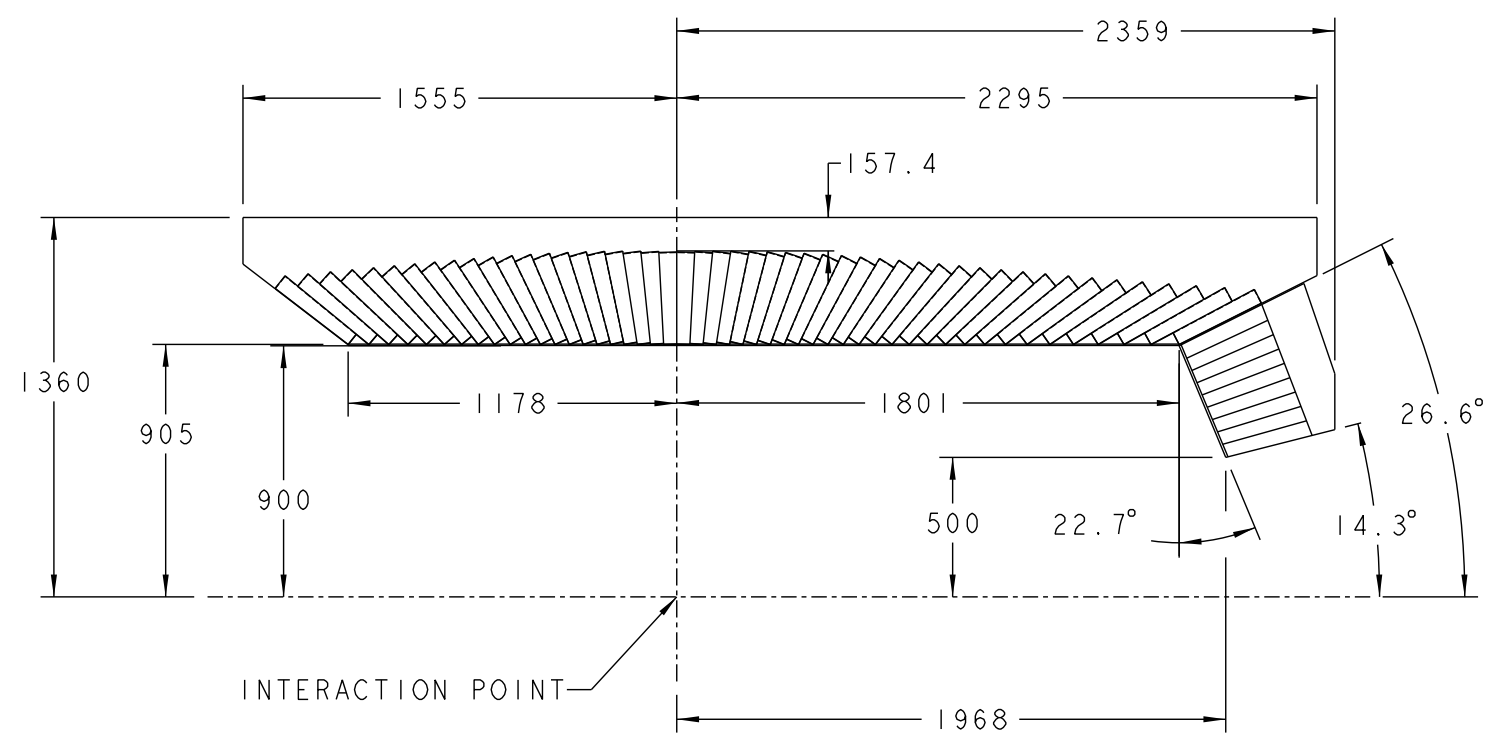

Figure 3.20: Diagram showing the electromagnetic calorimeter [14] (all dimensions shown are in millimeters). As with the other detector components shown in this chapter, the electromagnetic calorimeter is a full cylinder; the illustration provided here is only a side view. Also notice that the crystals are not arranged symmetrically about the interaction point, but rather offset so that more crystals are present in the forward region.

Scintillators, such as the crystals that will be used for the electromagnetic calorimeter, work by absorbing the energy of an incoming photon or particle and emitting light in an energy regime to which detector sensitivity is well matched. Typically photomultiplier tubes are used to detect the scintillation light, but instead the $B_{A} B_{A R}$ calorimeter will make use of smaller, less expensive silicon PIN photodiodes in conjunction with wavelength shifting fluorescent dyes. The purpose of the wavelength shifter is to shift the wavelength of the $\mathrm{CsI}(\mathrm{Tl})$ scintillation light $(560 \mathrm{~nm})$ to match the sensitive range of the photodiode (960 nm). After the scintillation light has been collected by the PIN photodiodes, the pulse that is observed is directly proportional to the energy of the 
incident photon or particle. Inorganic crystals such as $\mathrm{CsI}(\mathrm{Tl})$ are particularly well suited as scintillators because of their high density (stopping power) and high light output which leads to good energy resolution. Density of the scintillator material is very important because if the particle is not completely absorbed in the medium then the energy deposition that is measured is not the total energy of the particle, but rather only the amount lost while passing through the calorimeter. By the same token, the mass of the detector components that lie between the interaction point and the electromagnetic calorimeter must be kept low so that as little energy as possible is lost by the particles as they make their way to the scintillators, and the calorimeter is able to make an accurate measurement of the energy of the particles at the time of creation. When photons interact with metal they may convert to $e^{+} e^{-}$pairs that curl up in a magnetic field. The consequence of this conversion is that the energy that the initial photon was carrying does not reach the calorimeter, or, if the $e^{+} e^{-}$pairs do reach the calorimeter, they are detected by a different scintillator crystal than the rest of the decay products and are therefore not associated with the same event.

\subsubsection{Muon and Neutral Hadron Detector}

Surrounding the $B_{A} B_{A R}$ detector is a large iron return yoke that is instrumented to provide muon and $K_{L}^{0}$ identification. The ability to detect muons is important because knowing the energy or momentum and charge of the lepton will indicate whether a $B^{0}$ or a $\overline{B^{0}}$ decay has occurred. As mentioned in Section 3.2, a positively charged lepton or kaon will tag a $\bar{b}$ decay, while a negatively charged lepton or kaon will tag a $b$ decay. Working backwards, it is then possible to know the point in time when one of either of the $B$ mesons was in a definite state as a $B^{0}$ or $\overline{B^{0}}$.

The instrumented flux return (IFR) illustrated in Figure 3.21 is 24 layers of steel, 
each of which is $2.5 \mathrm{~cm}$ thick, which screen out pions by preventing pion "punchthrough", with resistive plate counters (RPCs) inserted between the plates. The principle behind the RPCs is similar to that of the drift chamber. When a charged particle passes through the chamber, it ionizes the gas, and a signal is measured on electrode plates made of 2 mm-thick Bakelite.

The iron structure also provides the external flux path for the magnetic field from the superconducting solenoid that will be mentioned in the next section. The reason that this detector assembly is called the "instrumented flux return" is that while the iron framework serves as the flux return, the interior space between the iron layers is instrumented with muon and neutral hadron detectors.

\subsubsection{Magnet Coil}

The $B_{A} B_{A R}$ magnet is a $1.5 \mathrm{~T}$ superconducting solenoid with a uniformity of $\pm 2 \%$ in the tracking region. The conductor is made of a superconducting niobium titanium (NbTi) cable which operates at a temperature of $20 \mathrm{~K}$. Due to the low temperatures

required for the operation of the superconducting magnet, a surrounding cryogenic system is also necessary. 


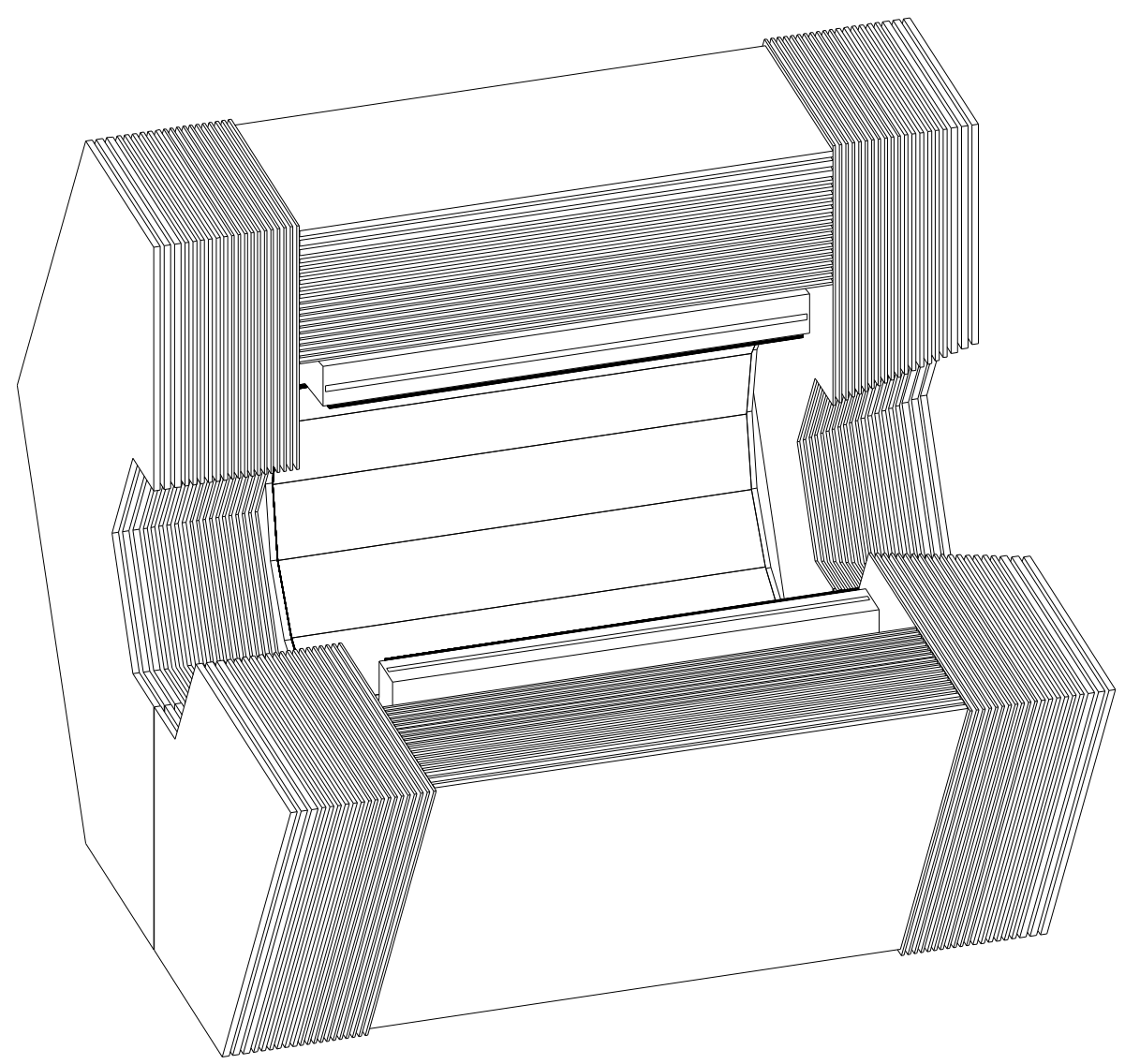

Figure 3.21: Diagram showing the instrumented flux return (IFR) [14]. In between the many iron plates that serve as the magnetic flux return, resistive plate counters are inserted to detect muons and neutral hadrons. 


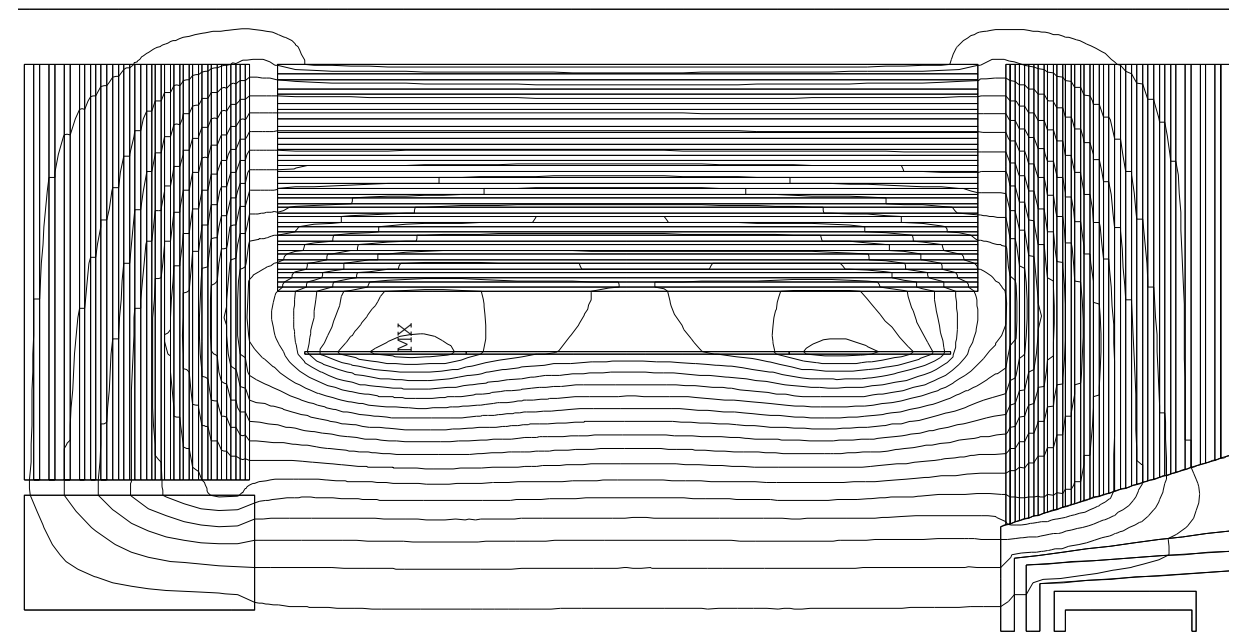

Figure 3.22: Schematic showing the magnetic field lines of the 1.5 T magnet [14]. The solenoid for the superconducting magnet is housed within the IFR shown in Figure 3.21. The horizontal field lines at the bottom of the diagram indicate good field uniformity in the region where the drift chamber will be located. 


\section{Chapter 4}

\section{Drift Chambers}

\subsection{Introduction}

The drift chamber is an instrument used in the detection of charged particles by means of observing ionization in the chamber medium (usually taken to be gaseous) induced by the particles as they traverse the chamber, interacting with the molecules within. Its early predecessors include the ionization chamber, the proportional counter and the Geiger-Müller counter, and more recently, the multi-wire proportional chamber. The basic underlying principle behind all of these devices, including the drift chamber, is the same.

\subsection{Ionization Detectors}

Consider a hexagonal container filled with gas, for example, one of the cells in the prototype drift chamber that will be discussed in Chapter 5. A wire running through the centre of the cell, the anode or sense wire, is maintained at a positive voltage relative to the surrounding cathode or field wires which create an electric field directed from the anode to the cathode (see Figure 4.23).

When a high energy particle enters the chamber, electron-ion pairs may be created when the particle collides with one of the atoms in the gas, knocking one of the electrons out from its orbit from around the nucleus,

$$
\text { particle }+X \rightarrow X^{+}+\text {particle }+e^{-} .
$$




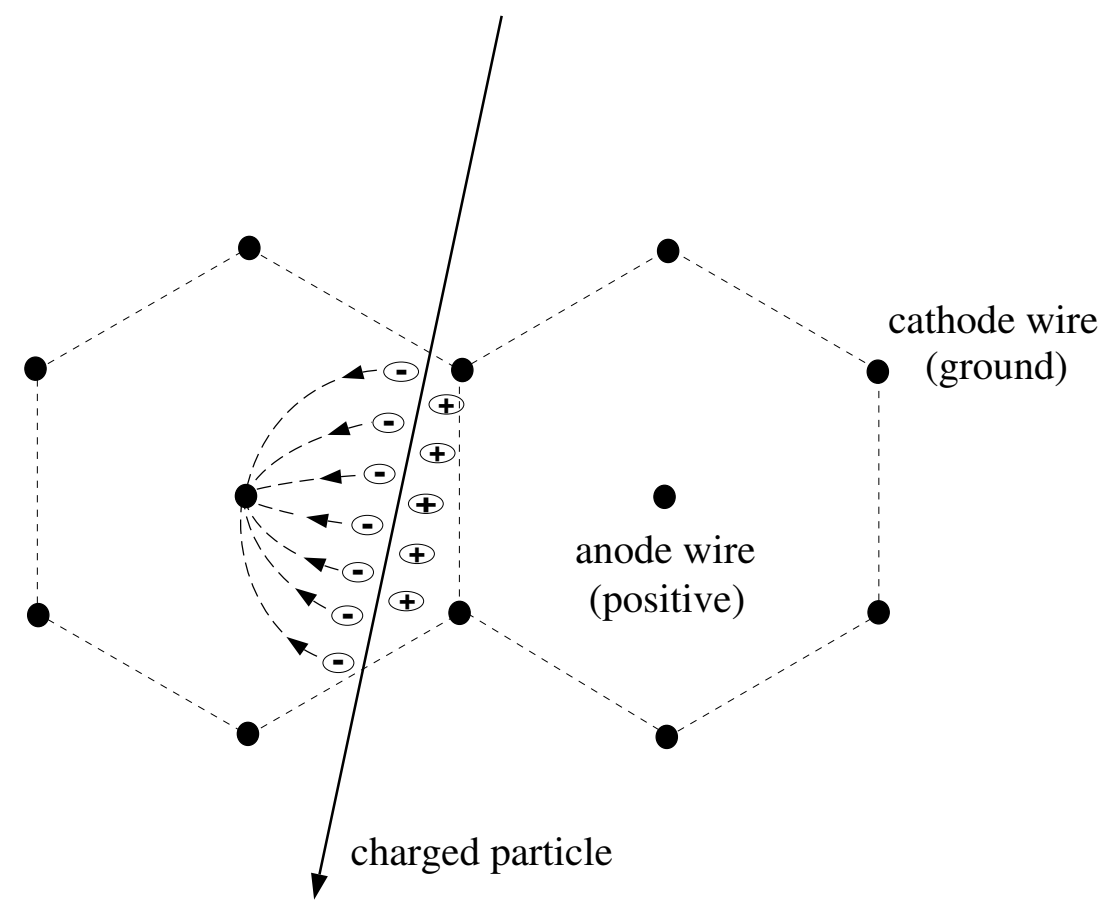

Figure 4.23: Schematic of a drift chamber cell. A charged particle is shown entering the cell volume whereupon it proceeds to ionize the drift chamber gas. The electrons freed in the ionization process accelerate toward the anode wire due to the potential difference between the anode and cathode wires. The dashed lines are included only to indicate the hexagonal shape of the cell; no physical boundary exists other than the cathode wires to define the cell geometry.

This is the process known as ionization.

In the presence of the electric field, negatively charged electrons will accelerate toward the nearest anode wire because it is held at a positive potential relative to the cathode wires at the edge of the cell boundary, while the remaining ions will migrate toward the cathode wires which are maintained at a relatively negative potential.

The anode wires in a drift chamber have high voltage applied to them so that when the electron-ion pairs are created and the electrons accelerate toward the anode wire, each of the primary electrons is then able to gain sufficient energy due to the high 
acceleration to further ionize the chamber gas molecules that lie in its path, freeing secondary electrons in the process. These secondary electrons are then able to produce more ionization and the process escalates into what is called an ionization avalanche. If a sufficiently large number of electrons are contained in the avalanche and are collected at the sense wire (that is, enough charge), then a signal may be detected. The number of electrons at each step - primary ionization, secondary ionization, leading up to and including the electron avalanche that collects on the sense wire and ultimately the height of the pulse that is measured - is directly proportional to the energy of the charged particle. The gas gain is defined to be the ratio of the number of electrons in the avalanche that arrive at the sense wire to the number of initial primary ions, and is typically on the order of $10^{6}$.

The time taken from the first ionization, $t_{0}$, to signal readout, $t$, may be measured using an external trigger that indicates the point of a certain event in time. Using this information, the drift time can be deduced. In the case of the prototype drift chamber that will be discussed in Chapter 5, a scintillation counter placed above the chamber. Furthermore, if the electrons drift toward the anode wire with a known velocity, $v_{d}$, called the drift velocity, then the position of the particle when it passed through the cell is also known:

$$
x=\int_{t_{0}}^{t} v_{d} d t
$$

In practice, it is desirable to have a constant electric field and a constant drift velocity so that there is a linear relationship between time and distance of the form $x=v_{d}(t-$ $\left.t_{0}\right)$. Therefore, two of the key ingredients to a successful drift chamber include good knowledge of time and a well understood drift velocity. It should be made clear that the incoming charged particle is not absorbed in the process of ionization, but rather leaves a trail of ionization behind it. 
How well the position of the particle is known and detection efficiency is primarily governed by the choice of gas and the cell size and geometry used in the chamber.

\subsection{Gas Properties}

There are numerous gas properties that dictate the suitability of a gas for a particular drift chamber and even motivate design features. A few of those characteristics include the type of gas (whether it should be monatomic, diatomic or even more complex), drift velocities and how this quantity for various gases reflects on chamber stability, and gas diffusion.

\subsubsection{Choice of Gas Type}

There are a number of factors to be considered when choosing a drift chamber gas. In Chapter 3, the need to reduce the total amount of material in the chamber was outlined and in order to measure low momentum particles, and obtain $d E / d x$ information for particles that will not reach the particle identification system, multiple scattering is required to be as small as possible. For now though, more generic requirements will be discussed which are common to drift chambers in general.

For a chamber that operates at room temperature and atmospheric pressure, there are only a few elements that exist in a gaseous state such as oxygen $\left(\mathrm{O}_{2}\right)$, nitrogen $\left(\mathrm{N}_{2}\right)$, fluorine $\left(\mathrm{F}_{2}\right)$, and the noble gases, helium (He), neon (Ne), argon (Ar), krypton (Kr), and xenon $(\mathrm{Xe})$. Notice that the noble gases are monatomic while oxygen, nitrogen and fluorine are diatomic and therefore more complex. If the size of the molecule is not an issue, even more complex gas mixtures would be possible.

However, general requirements that dictate the choice of gas include having a low 
operating anode voltage. Avalanche multiplication occurs in noble gases such as argon and helium using much lower anode voltages than gas mixtures involving complex molecules because of the many non-ionizing energy dissipation modes complex molecules have available. For instance, an excited noble gas atom can return to its ground state only through a radiative process in which a photon is emitted, which in turn can lead to further ionization avalanching. Complex molecules, on the other hand, can dissipate energy through collisions and disassociation into smaller molecules which the simple noble gases cannot do.

At high voltages, there is a problem with having only noble gases present in the chamber. Ultraviolet photons that are released in the process of de-excitation of an excited noble atom, $X$,

$$
e^{-}+X \rightarrow X^{*} \rightarrow X+\gamma
$$

are typically much greater in energy (11.6 eV for argon, $19.8 \mathrm{eV}$ for helium) [16] than the work function ${ }^{1}$ of any metal cathode (4.82 eV for a gold plated cathode) [17]. Unwanted photoelectrons from the cathode are produced when struck by these high energy electrons, which in turn begin the repeated ionization process as they move toward the anode. The remedy is to add a small amount of quencher gas, a complex molecule such as carbon dioxide $\left(\mathrm{CO}_{2}\right)$ or iso-butane $\left(i-\mathrm{C}_{4} \mathrm{H}_{10}\right)$ which prevents this from occurring in a number of ways. First, since complex molecules have a large number of non-radiative excited states (rotational and vibrational), they can absorb a wide range of photon energies and in doing so prevent avalanching by removing the ultraviolet radiation from the de-excitation process before it reaches the cathode, without contributing an electron in the process. Other ways complex molecules help dissipate energy include colliding with other gas molecules or through dissociation.

\footnotetext{
${ }^{1}$ The work function of a metal is the energy that a photon needs in order to just free one of the outermost electrons.
} 
There is one significant side effect to using a quencher gas, and that is wire aging. The quenching process discussed above may occasionally lead to polymerization in which liquid or solid polymers deposit on the chamber wires which seriously affects the operation of the drift chamber. If there are a lot of high energy ultraviolet photons in the chamber, then positive ions are produced faster than the cathode can neutralize them and the excess builds up, coating the field wires. Cleaning these wires is one solution, but other studies are in progress to determine which gases show the least aging effects.

As long as the dominant process is ionization, the number of electrons that arrive at the sense wire is proportional to the energy of the incoming particle. However, due to a process called electron attachment, not all of the the electrons that are liberated through ionization remain free until they reach the anode. This mechanism consists of the capture of a free electron produced through ionization by an electronegative atom ${ }^{2}$, $X$, to form negative ions,

$$
e^{-}+X \rightarrow X^{-}+\text {energy }
$$

rather than the desired ionization process shown in Equation 4.104 .

If, by the addition of an electron, the outer electron shell surrounding the nucleus becomes full, then the negative ion, $X^{-}$, formed is stable and is in a lower energy state than the original species, $X$, which means that energy is released in the above reaction. For noble gases however, the energy released is negative, which implies that in order for the electron attachment process to occur for a noble gas, energy must be provided. Therefore, a choice of argon or helium as the primary drift chamber gas minimizes the loss of electrons due to electron attachment, and the height of the pulse detected at the anode is a truer measure of the energy of the incoming particle.

\footnotetext{
${ }^{2}$ Electronegative atoms have an almost full outer electron shell and will capture a free electron in order to fill that shell, thereby becoming more stable.
} 
The Penning effect is another important process to consider. In this case though, the reaction mechanism enhances ionization which occurs between molecular gases and noble gases and between two noble gases. Certain atoms may be excited to metastable, relatively long-lived, states that de-excite when a collision occurs with a second atom causing ionization in the latter. For example, the following reactions might occur:

$$
\begin{gathered}
\mathrm{He}^{*}+\mathrm{CO}_{2} \rightarrow \mathrm{He}+\mathrm{CO}_{2}^{+}+e^{-} \\
\mathrm{Ne}^{*}+\mathrm{Ar} \rightarrow \mathrm{Ne}+\mathrm{Ar}^{+}+e^{-}
\end{gathered}
$$

Because of the Penning effect, drift chambers containing noble gases can operate at slightly lower voltages.

In light of these processes, a noble gas would seem to be a good choice for the primary chamber medium, with additions of complex molecules such as $\mathrm{CO}_{2}$ or $i-\mathrm{C}_{4} \mathrm{H}_{10}$ to act as quenching impurities.

\subsubsection{Drift Velocity}

The drift velocity, $v_{d}$, of an electron superimposed on random thermal motion and in the presence of an electric field, $E$, is given by the expression [18]:

$$
v_{d}(x)=\frac{2}{3} \frac{e l}{m_{e} \bar{u}} E(x)
$$

where $e$ is the charge of the electron, $l$ is the mean distance that the electron travels before colliding with an atom or molecule in the chamber medium (called the mean free path), and $\bar{u}$ is the mean thermal velocity of the electron liberated by the incoming ionizing particle. The factor multiplying $\mathrm{E}$ is known as the mobility of the electrons. Since $\bar{u}$ is higher in a gaseous medium than in say, a liquid, the mobility will be lower, leading to a lower drift velocity, $v_{d}$. Typical drift velocities in helium- or argon-based gases are on the order of $10-25 \mu \mathrm{m} / \mathrm{ns}$ which is much lower than typical random thermal 
velocities which are in the $1000 \mu \mathrm{m} / \mathrm{ns}$ range at room temperature. Low drift velocities lead to large drift times and thus better position resolution because of the improved timing resolution, while high drift velocities are important in experiments where the count rates are expected to be high and therefore deadtime becomes important.

To optimize chamber stability, a gas exhibiting drift velocity saturation at reasonably low electric fields should be chosen. Figure 4.24 shows a number of different examples where electron drift velocities are relatively constant over a range of electric field intensities for various gas mixtures. When operated in this plateau region, the drift velocity is then less sensitive to inhomogeneities in the electric field and environmental variables such as temperature and pressure. However, since drift velocity is very sensitive to "impurities" within the gas, great care must be taken to ensure that, once the plateau region for a particular gas is found, impurities are not subsequently introduced that would cause a shift the plateau region away from the original high voltage settings. Figure 4.25 illustrates the effect of having a small amount of impurity present. Notice that not only does the drift velocity change when impurities are added, but so does the position of the saturation plateau.

\subsubsection{Electron Diffusion}

The limit on the spatial resolution of a drift chamber is governed predominantly by the diffusion of electrons in the chamber medium as they move toward the anode wire [16].

In the absence of an electric field, the electron-ion pair formed by the particle diffuses uniformly outward, from regions of high concentration to regions of low concentration from their point of creation. The concentration of electrons at a later position $x$ after diffusing a time $t$ is approximately Gaussian, and is given by the following 


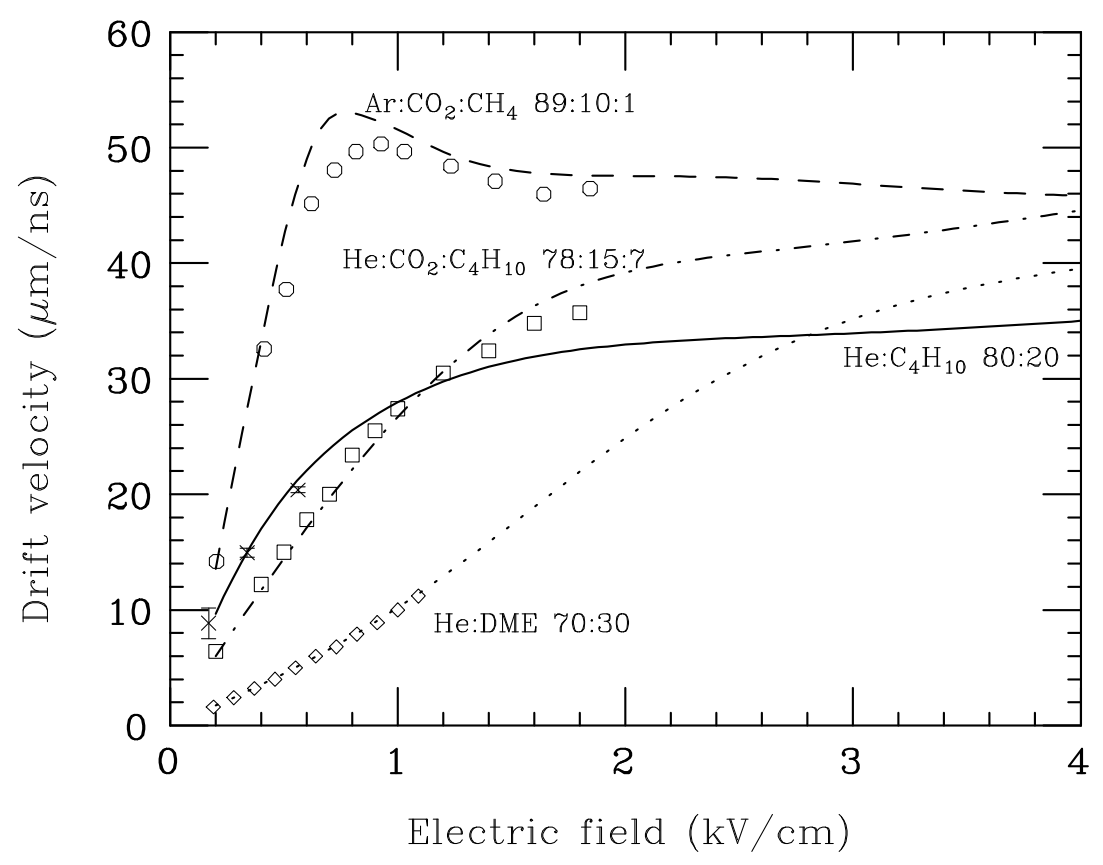

Figure 4.24: Calculated and measured drift velocities as a function of electric field with no magnetic field present [14]. In each case, the plateau region indicates that saturation has been reached, and the drift chamber will be least sensitive to changes in the electric field. Notice that different gas mixtures have different saturation points.

expression [16]:

$$
\frac{d N}{d x}=\frac{N_{0}}{\sqrt{4 \pi D t}} \exp \left(-\frac{x^{2}}{4 D t}\right)
$$

where $x$ is the distance from the point of ionization, $N_{0}$ is the initial number of electrons at $x=0$ and $t=0$, and $D$ is the diffusion coefficient which, from kinetic theory, is given by:

$$
D=\left(\frac{u^{2}}{3 \nu}\right)_{\text {average }}=\frac{1}{3} l \bar{u}
$$

where $\bar{u}$ is the average velocity of the electron and $\nu$ is the frequency of electron collisions with the chamber gas.

Comparing Equation 4.111 to the form of a Gaussian distribution, distributed about 


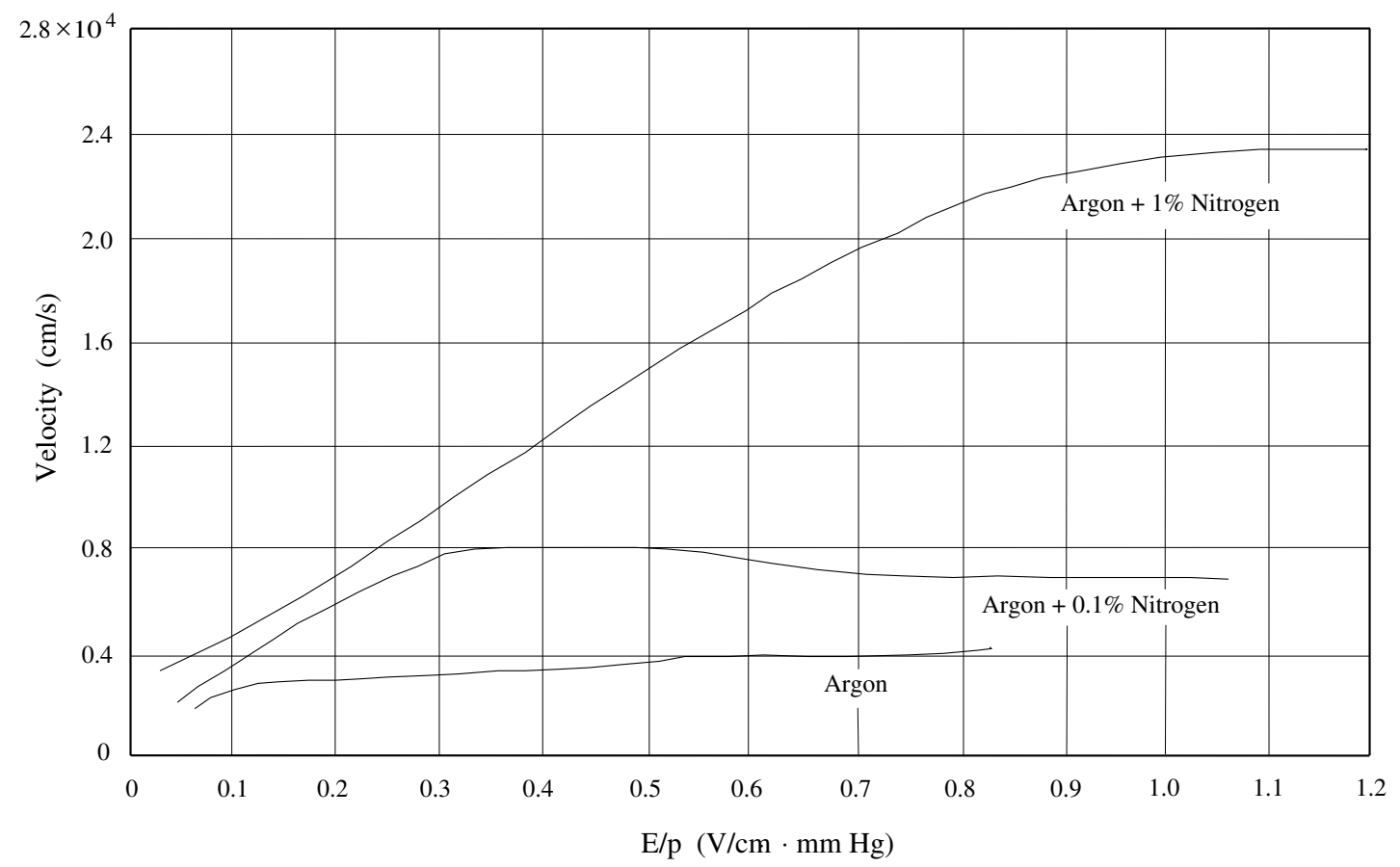

Figure 4.25: Drift velocity vs. electric field indicating the influence of nitrogen impurities in argon gas [19]. Notice the strong dependence on gas purity - the addition of $1 \%$ nitrogen to argon increases the drift velocity by a factor of 5 , as well as changing the position of the saturation plateau.

$x=0$ :

$$
y=\frac{1}{\sigma \sqrt{2 \pi}} \exp \left(-\frac{x^{2}}{2 \sigma^{2}}\right),
$$

the standard deviation of the electron distribution, or the spatial spread of electrons at $x$ is identified as:

$$
\sigma(x)=\sqrt{2 D t}=\sqrt{2 D \cdot \frac{l}{v_{d}(x)}}=\sqrt{\frac{m_{e} \bar{u}^{2}}{e E(x)}} .
$$

The dependence of spatial resolution on the size of the cell is now explicitly clear. The diffusion distribution varies as the square root of the drift length, which means that the longer the path the electron travels, the more diffuse the distribution will be due to an increase in the number of collisions. Ultimately, a high diffusion will adversely 
affect the spatial resolution of a drift chamber since the timing for a dispersed group of electrons becomes difficult to determine accurately. Therefore, a small cell size should give improved spatial resolution as well as avoid the use of very high voltages. With drift path lengths on the order of $1 \mathrm{~cm}$, spatial resolution values of less than $100 \mu \mathrm{m}$ are expected [20].

Figure 4.26 shows the diffusion of electrons from a layer as a function of time. With an electric field present, the mean of the electron distributions is shifted without affecting the relative shapes.

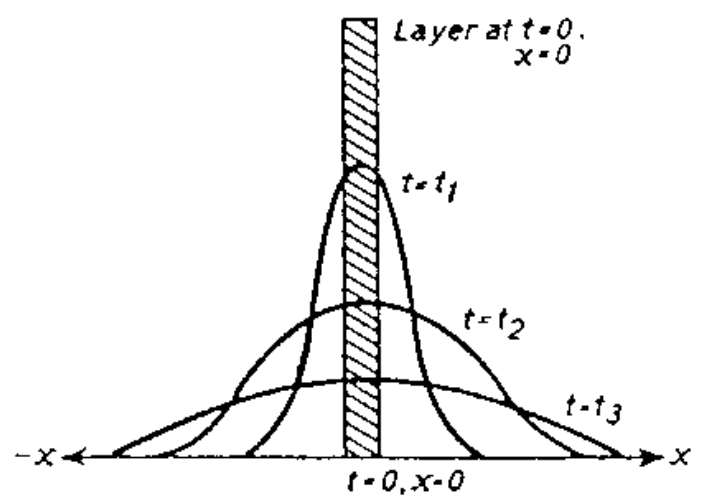

(a)

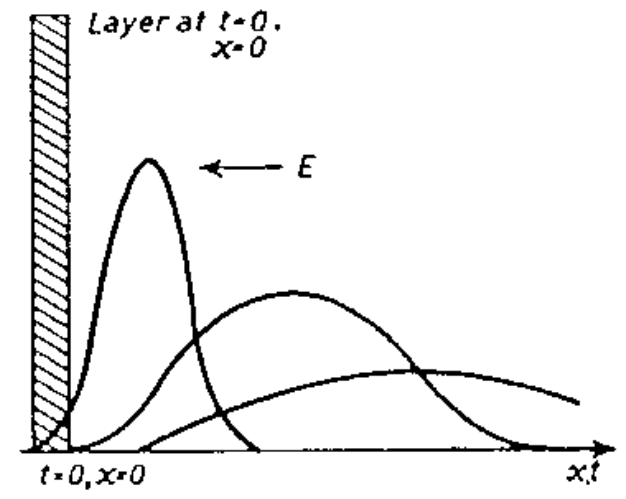

(b)

Figure 4.26: Shown is the diffusion of electrons (a) with no electric field present, and (b) in the presence of an electric field [18]. Electrons are localized at $t=0$ (indicated by the shaded region) and spread out spatially due to diffusion as time progresses.

\subsection{Wire Configurations}

Having information about where the particle may have passed through a single cell is not very useful. Many cells spanning the area of interest and stacked one on top of the other are needed in order to resolve spatial coordinates accurately. The trajectory or track of the particle as it passes through many layers is obtained by utilizing the array 
of distances from the sense wires.

In a drift chamber where all wires are axial, the $z$ component along the wire direction is often left undetermined because it is not straightforward to distinguish between a particle passing through the chamber at two different points along the length of the chamber. The solution is often to include a layer (or number of layers) of stereo wires. These wires are strung at an angle with respect to the other wires which are strung straight across (referred to as axial wires) as shown in Figure 4.27. Typical stereo angles are small, usually being less than $10^{\circ}$.

The axial wires are used to measure $x$ and $y$ as before, but knowing which axial wire along with which stereo wire was hit means that the $z$ position can now be deduced. Therefore, with an appropriate wire arrangement, the path of the particle can be reconstructed in 3 dimensions.

\subsection{Behaviour of a Drift Chamber in a Magnetic Field}

Still more information can be gained by using a drift chamber in the presence of a magnetic field. In a region of uniform magnetic field, $\mathbf{B}$, the Lorentz force, $\mathbf{F}$, is given by:

$$
\mathbf{F}=q \mathbf{v} \times \mathbf{B}
$$

continually deflects charged particles that have a component of velocity perpendicular to $B$ causing them to follow a circular path. The radius of curvature of this helical path (circular in the $x-y$ plane and linear in $z$ ) is given by

$$
r=\frac{m v \sin \theta}{q B}=\frac{p \sin \theta}{q B} \rightarrow p_{t}=r q B
$$

where $q$ is the charge of the particle, and $p_{t} \equiv p \sin \theta$ its transverse momentum. Hence, momenta of particles of a known charge can be determined by measuring the radii of 


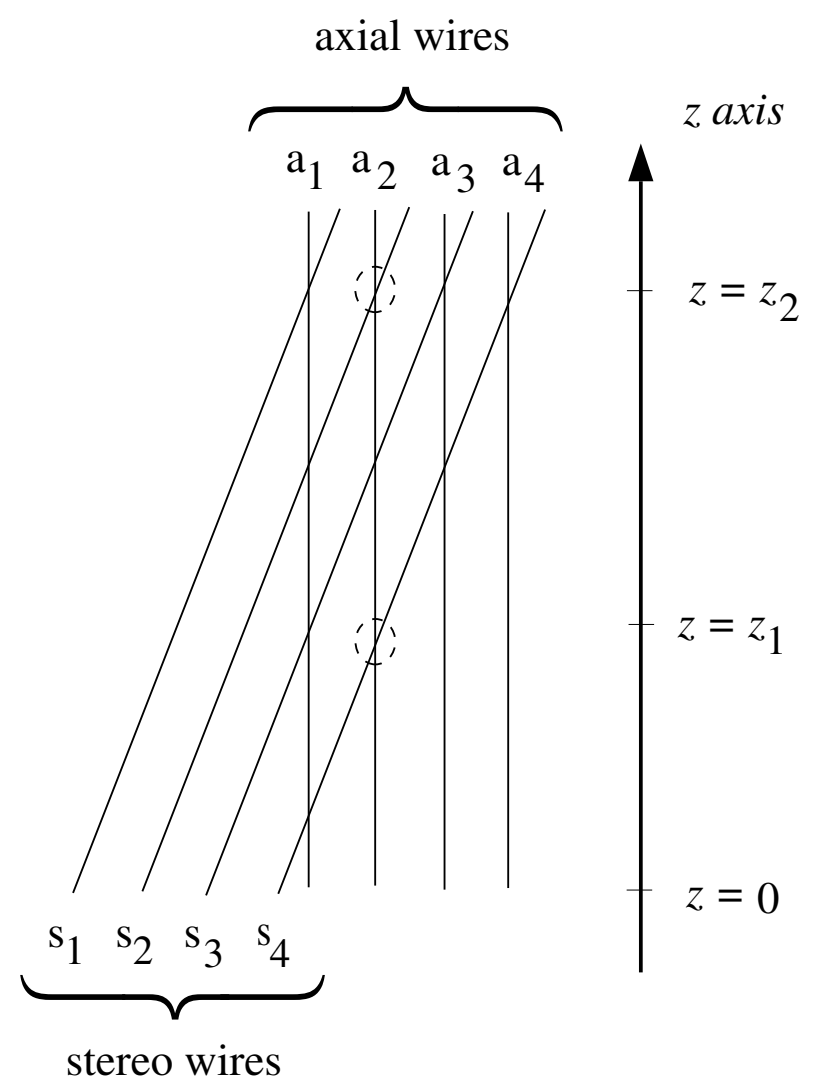

Figure 4.27: Diagram illustrating axial and stereo wire configurations. Using both the axial and stereo wires, it is possible to gain information about all 3 coordinates of the track. Knowing only that a hit was recorded on axial wire $a_{2}$ is not enough to determine the position in the $z$ direction. However, using the additional information provided by the stereo wires, it is possible to infer the $z$-position. For instance, if a hit is detected in axial wire $a_{2}$ and stereo wire $s_{4}$, then $z=z_{1}$; on the other hand, if a hit is detected in wires $a_{2}$ and $s_{2}$, then $z=z_{2}$. 
curvature of tracks in a known magnetic field.

However, since the presence of a magnetic field affects both the path of the particle as well as the drift velocity, precise knowledge of the magnetic field is necessary in order to correlate drift time with position. 


\section{Chapter 5}

\section{Prototype Drift Chamber}

\subsection{Introduction}

In order to test some of the features of the proposed $B_{A} B_{A R}$ drift chamber, such as the electrostatic stability of a long chamber, small cell design, wire material and various gas mixtures, a full-length prototype version was constructed at SLAC. The efficiency and resolution analysis outlined in Chapters 7 and 8 used data that were collected using this prototype chamber.

\subsection{Prototype Geometry}

The prototype chamber is $2.5 \mathrm{~m}$ in length and includes 72 sense (anode) wires of $20 \mu \mathrm{m}$ gold plated tungsten and 512 field (cathode) wires of $55 \mu \mathrm{m}$ unplated aluminum arranged in 12 layers or 3 superlayers (4 layers per superlayer) as shown in Figure 5.28. Two of the superlayers have axial wires which are strung straight across the length of the chamber, and one superlayer which has stereo wires that are strung at an angle of $50 \mathrm{mrad}\left(\approx 3^{\circ}\right)$ with respect to the axial wires.

\subsection{Gases}

Due to the requirement that the inner tracking components, including the drift chamber, have as little mass as possible, the $B_{A} B_{A R}$ drift chamber group decided to investigate a low mass (or atomic number, $Z$ ) gas such as helium. One of the main purposes 


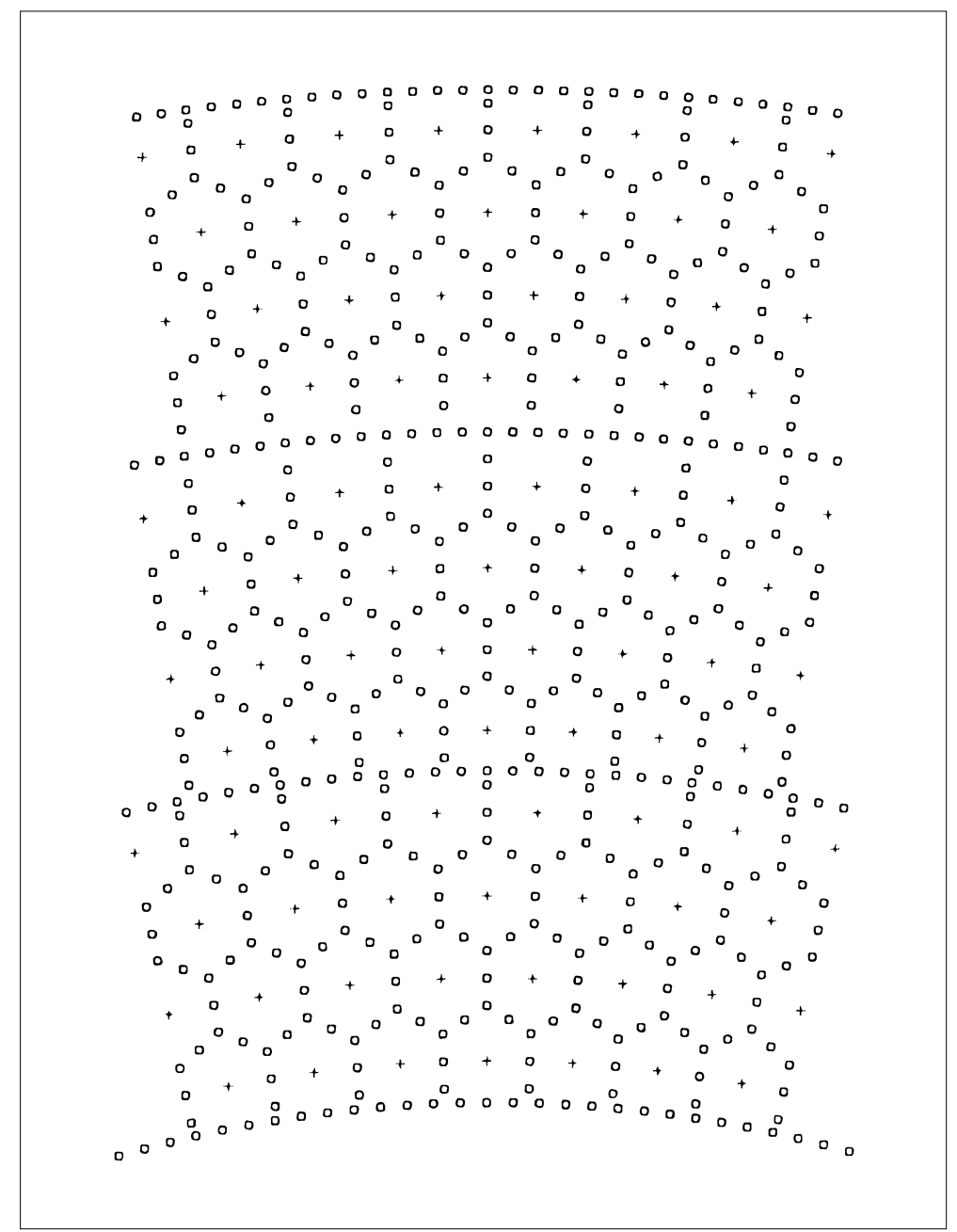

Figure 5.28: Diagram showing the wiremap of the prototype drift chamber [21]. The circles in the drawing represent cathode wires, while the crosses indicate the position of the anode wires. The cells in the middle of the superlayers are hexagonal in shape, whereas, at the superlayer boundaries the cells are pentagonal. Each side of the cell is approximately $1 \mathrm{~cm}$. The top and bottom layers are composed of axial wires, while the middle layer has stereo wires that are strung at an angle of $50 \mathrm{mrad}$ with respect to the axial wires. A layer of field wires is inserted between superlayers to allow cells to change shape and relative position from one superlayer to the next. 
for building the prototype was to test whether helium-based gases could perform as well as the more traditional argon-based gas mixtures. So far, four gas mixtures have been tested in the prototype, namely: $\mathrm{Ar}: \mathrm{CO}_{2}: \mathrm{CH}_{4}[89: 10: 1], \mathrm{He}: \mathrm{CO}_{2}: i-\mathrm{C}_{4} \mathrm{H}_{10}$ [83:10:7], He: $i-\mathrm{C}_{4} \mathrm{H}_{10}$ [80:20], He: $\mathrm{C}_{3} \mathrm{H}_{8}$ [70:30]. As discussed in Chapter 4, a number of properties are important when choosing a drift chamber gas, including the number of primary ions. One advantage of using helium is that it has approximately 50 times the radiation length of argon, however, it also has fewer primary ions released upon ionization. On one hand, a large radiation length contributes to lower mass and therefore higher momentum resolution, but, on the other hand, having fewer primary ions released leads to poorer resolution. Furthermore, the helium-based gases tend to be slower; that is, the electrons, once freed from a helium gas mixture, typically drift toward the anode at a slower rate than the corresponding argon gases. This is an advantage because having a gas mixture with a slower drift velocity leads to larger drift times and thus better position resolution because of the improved timing resolution. One last requirement of the drift chamber is that it must provide a means of identifying particles with low momentum that will not reach the DIRC or ATC particle identification systems. Particle identification in the drift chamber relies on energy deposition in the chamber gas. The higher the density of the gas, the more energy is deposited and the better the identification resolution. Argon-based gases tend to be better suited to perform this task due to their high mass, but based on minimum ionization calculations, the helium gas mixtures under study are expected to give comparable $d E / d x$ resolutions. Table 5.3 provides a summary of the various properties of the gases tested.

The analysis that follows in Chapters 7 and 8 examines whether or not there is a trade-off between radiation length and performance factors like efficiency and spatial resolution. 


\begin{tabular}{|l|c|c|c|c|c|}
\hline Gas Mixture & Ratio & $\begin{array}{c}\text { Radiation } \\
\text { Length, } \\
(\mathbf{m})\end{array}$ & $\begin{array}{c}\text { Primary } \\
\text { Ions/cm }\end{array}$ & $\begin{array}{c}\text { Drift } \\
\text { Velocity, } v_{\boldsymbol{d}} \\
(\mu \mathbf{m} / \mathbf{n s})\end{array}$ & $\begin{array}{c}d E / d x \\
\text { Resolution } \\
(\mathbf{\%})\end{array}$ \\
\hline \hline $\mathrm{Ar}: \mathrm{CO}_{2}: \mathrm{CH}_{4}$ & $89: 10: 1$ & 124 & 23.6 & 49 & 7.3 \\
$\mathrm{He}: \mathrm{CO}_{2}: i-\mathrm{C}_{4} \mathrm{H}_{10}$ & $83: 10: 7$ & 963 & 13.8 & 19 & 8.5 \\
$\mathrm{He}: i-\mathrm{C}_{4} \mathrm{H}_{10}$ & $80: 20$ & 807 & 21.2 & 22 & 6.9 \\
$\mathrm{He}: \mathrm{C}_{3} \mathrm{H}_{8}$ & $70: 30$ & 733 & 24.1 & 24 & 6.5 \\
\hline
\end{tabular}

Table 5.3: Table of calculated properties for various gas mixtures at atmospheric pressure and $20^{\circ} \mathrm{C}$ [14]. The most important thing to notice is that the helium-based gases have a much longer radiation length than their argon-based counterpart, which means that particles passing through them interact less often and lose less energy.

\subsection{Setup}

The detection process begins when a cosmic muon passes through the top scintillator above the prototype chamber, as illustrated in Figure 5.29. As the cosmic ray passes through the detector volume, it has the opportunity to ionize the chamber gas particles, the electrons from which migrate toward the anode wire as described in Chapter 4. Once the particle traverses the entire detector space, it can then pass through two remaining scintillator paddles located below the chamber. In order for an "event" to occur, there has to be a coincidence between all three scintillators, indicating that a track passed through the top and made it all the way to the bottom. The size of the scintillators restricts the incoming cosmic rays to be very close to vertical in order to simplify the subsequent analysis.

\subsubsection{TDCs}

The time interval between when the particle passes through the scintillator and when the pulse is detected at the anode wire is measured using a time-to-digital converter (TDC) module. A TDC works by using a "start" signal to initiate counting on a 


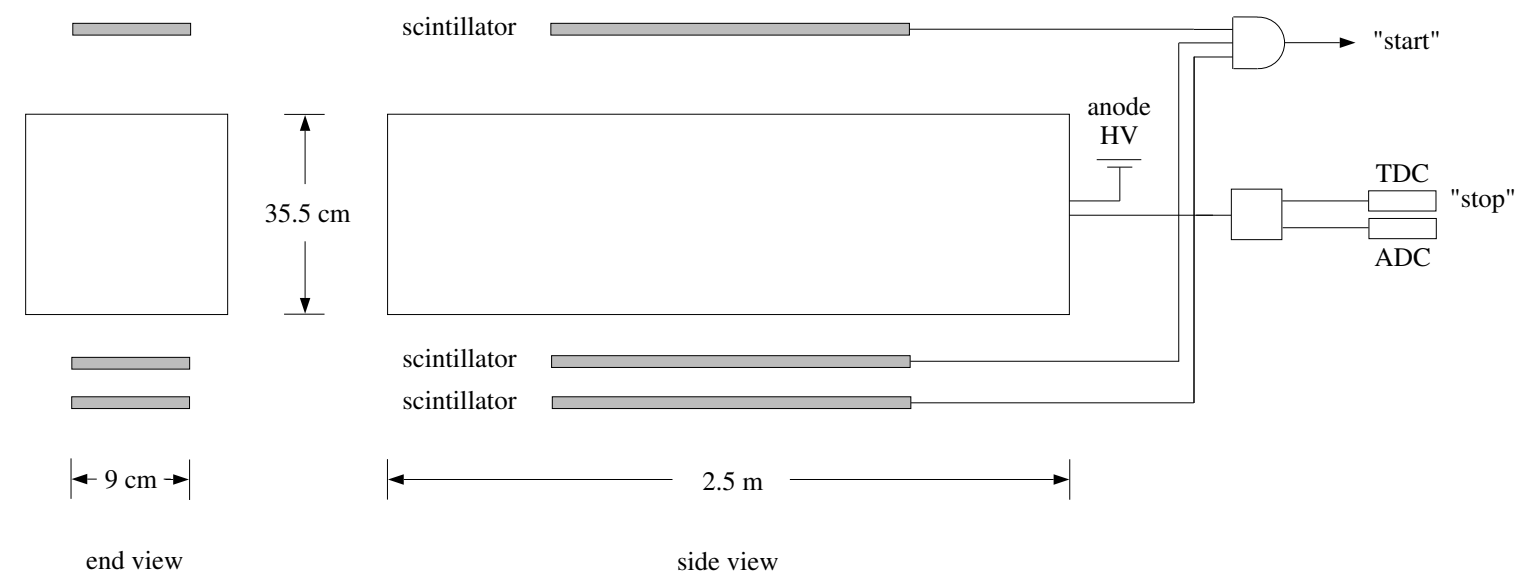

Figure 5.29: Diagram showing the prototype drift chamber and the positioning of the cosmic trigger scintillators.

constant frequency oscillator (or clock) and "stop" signal to end the counting interval, which is measured in channels. The more channels associated with a TDC, the better the time resolution. In the case of the prototype, a signal from the top scintillator starts the TDC, and a sufficiently large pulse from the anode wire stops it. The particular TDC modules used in this setup were LeCroy CAMAC 2228A 8 channel TDCs.

\subsubsection{ADCs}

An analog-to-digital converter (ADC) module measures the integrated amplitude of an input analog pulse and converts that value to a digital number. The ADCs used in this experiment were charge sensitive ADCs which means that the digital output is proportional to the charge deposited on the anode wire, and therefore the number of electrons. The ADC modules used in the prototype were LeCroy CAMAC 2249W 12 channel ADCs. 


\subsubsection{Data Collection}

The data were collected using an IBM RS6000 workstation, which runs the online data aquisition software. 


\section{Chapter 6}

\section{Track Fitting}

\subsection{Introduction}

In Chapter 5 the experimental setup was presented - the chamber itself with the axial and stereo wire configurations, the data acquisition system, including the ADC and TDC modules, as well as the scintillator paddles that were used in conjunction with the TDCs to provide time information. This chapter is devoted to a discussion of how this equipment gathers information and how the data are analyzed ${ }^{1}$ after they are obtained.

As far as this thesis is concerned, two of the most important studies that the prototype can be used to perform are spatial resolution and efficiency studies for various gases. The aim of the spatial resolution studies is to determine how well the position of the track within the chamber can be determined and compare this quantity between the argon- and helium-based gases. Recall from Chapter 4 that knowing the trajectory (position as a function of time) of a particle track well is equivalent to knowing the momentum of the particle well if the magnetic field is also well understood, which means that the most desirable gas must allow for good spatial resolution. The second study looks at the efficiency of the chamber for various gases; that is, are certain gases better than others at identifying regions of the chamber where a particle has passed through? In order to know the efficiency of the chamber, the trajectory of the particle

\footnotetext{
${ }^{1}$ The track finding and fitting code outlined below was developed by Steve Schaffner at SLAC.
} 
must be precisely known. Clearly, in both cases, it is essential that the path of the particle be known very well within the chamber. To understand how accurately the trajectory of a particle within the drift chamber can be known, an understanding of how well tracks are found and fit is crucial; therefore, a quick overview of the track fitting code is in order.

\subsection{Data Collection and Storage}

Raw prototype data is stored in Cheetah [22] format as wires hit, ADC channel counts, TDC channel counts. Cheetah is a data management system that was developed and is used at SLAC.

\subsection{Hardware Preliminaries}

In order to use the prototype drift chamber to make track measurements, information about the time it takes for a particle to ionize the chamber gas, as well as information about the charge collected on the sense wire must be known. In the section that follows, TDC calibrations and $t_{0}$ offsets are discussed with regard to time information, while data from the ADC modules are presented that indicate the pulse heights (charge collection) associated with the various gases tested.

\subsubsection{TDC Calibrations}

In the first stage of the track finding and fitting algorithm, the TDCs are calibrated, which means that a conversion between TDC channel number and time is found. Recall from the discussion of drift chambers in Chapter 4 that good knowledge of time is one of the key ingredients to good spatial resolution. TDCs act like a stopwatch, but rather than measuring the duration of an event in seconds, they keep track of time in channel 
numbers, so in order to be useful, the calibration of TDC channels in terms of time must be known.

The calibration itself involves sending a string of 8 pulses in time spacings of every $0.1 \mu \mathrm{s}$, as shown in Figure 6.30. The plot of TDC channel versus time is then fit to a straight line to give the conversion of TDC channel number to time. Notice in the figure that the relationship between TDC channel number and time is indeed linear.

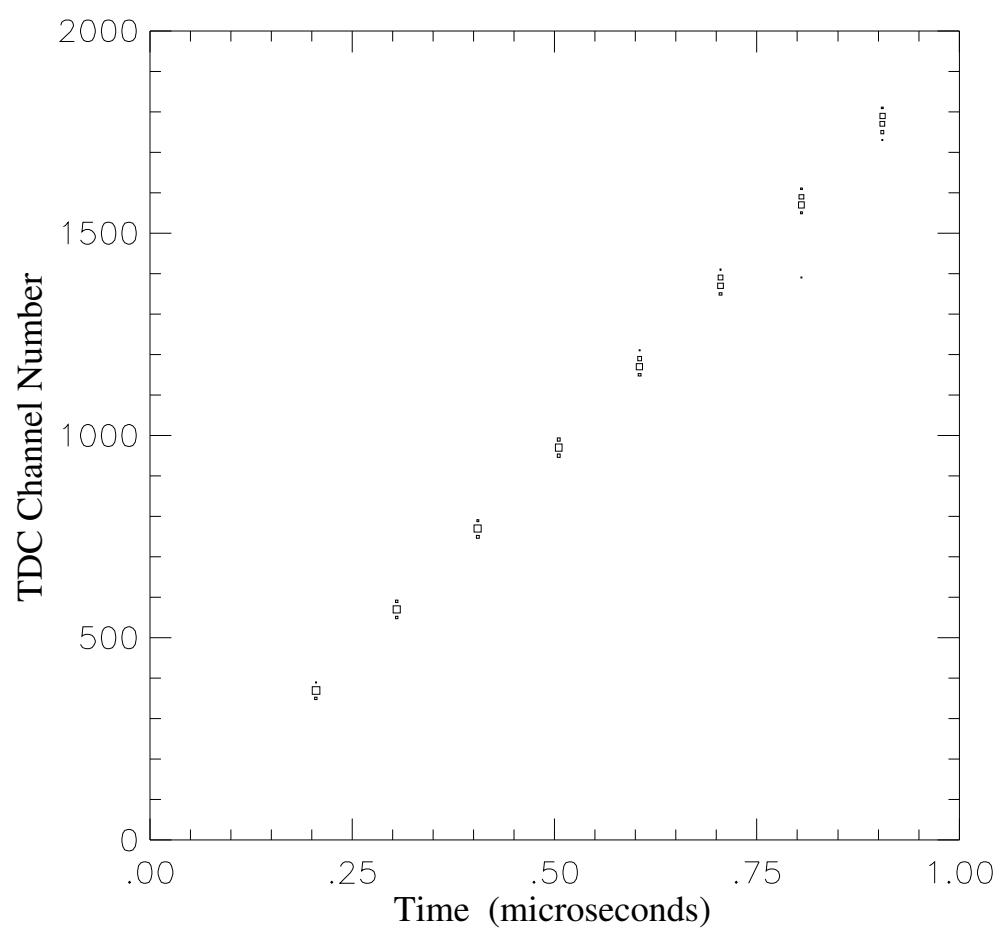

Figure 6.30: TDC channel number vs. time. This plot shows the linear relationship between TDC channel number and time.

The prototype drift chamber detects cosmic muons (see Chapter 5 for more details on the experimental setup). Recall that the time delay measured between when a cosmic ray ionizes an atom in the chamber and when the charge collected on the sense wire has exceeded a certain threshold level is the drift time of the ionized electrons in the gas mixture. As a cosmic muon passes through the chamber, it leaves a stream 
of ionized gas when it collides with a number of the atoms in the chamber gas, giving one or more of the electrons in each atom enough energy that they are able to leave the atom entirely. Due to the potential difference between the field and sense wires in the chamber, the electrons slowly migrate (the speed of the migration, or drift velocity, depends on the gas mixture used) to the positively charged anode wire, while the remaining ions make their way toward the negatively charged cathode wires surrounding the anode. As the electrons migrate to the sense wire, they are accelerated and in turn are able to ionize other gas atoms which lie in their path and the process escalates to a point where an "avalanche" of electrons is collected at the wire (see Chapter 4 for a general discussion on the workings of a drift chamber). The number of electrons that reach the wire must be above the TDC threshold if the pulse of electrons is to be detected and measured. If the number of electrons is too low, insufficient charge will collect at the sense wire and no pulse of electrons will be recorded.

\subsubsection{ADCs}

The purpose of an analog-to-digital converter (ADC) is to take the information contained in an analog current pulse signal, for example, and convert in to the equivalent digital form. Below is a typical histogram of ADC pulse height per hit (see Figure 6.31) which has had the zero-offset pedestal counts from each wire subtracted from it.

The noise at the "tail" of the distribution is excluded at ADC pulse height $\geq 1800$ and the mean of the distribution is taken to be representative of the pulse shape. The various gas mixtures are compared by plotting the logarithm of the mean pulse height versus high voltage as shown in Figure 6.32.

In Figure 6.32, it is evident that the mean pulse height has an exponential dependence on the anode high voltage. The height of the line on the plot is an indication of the number ion pairs produced in the gas mixture. Despite the fact that the number of 


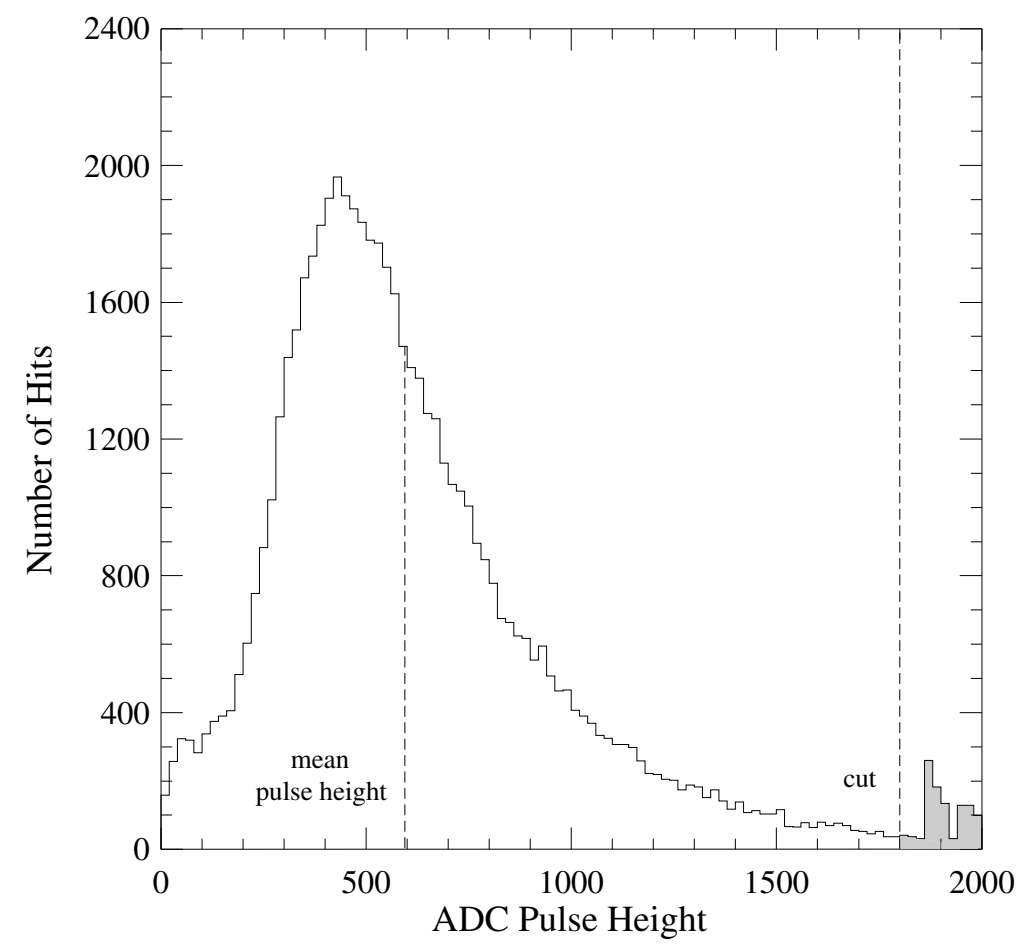

Figure 6.31: Histogram of $\mathrm{ADC}$ pulse height per hit using $\mathrm{He}: i-\mathrm{C}_{4} \mathrm{H}_{10}$ [80:20] gas at an anode voltage of $1850 \mathrm{~V}$. The noise at the far right of the distribution will be excluded when comparisons between the four gases are made.

primary ions per unit track length for $\mathrm{He}: i-\mathrm{C}_{4} \mathrm{H}_{10}[80: 20]$ gas is significantly lower than the other gas mixtures tested (see Table 5.3), its pulse height is very comparable to that of the $\mathrm{Ar}: \mathrm{CO}_{2}: \mathrm{CH}_{4}[89: 10: 1]$ and $\mathrm{He}: i-\mathrm{C}_{4} \mathrm{H}_{10}[80: 20]$ gas mixtures. It is unknown whether or not the Penning effect discussed in Chapter 4 is responsible for this increase in pulse height. On the other hand, $\mathrm{He}: \mathrm{C}_{3} \mathrm{H}_{8}[70: 30]$ gas has the most primary ions per unit track length, but it has a measured pulse height that is considerably lower than the other gas mixtures. Although this result is in general agreement with the results found by P.R. Burchat et al. [23] in their comparison of helium-based gases to Ar: $\mathrm{CO}_{2}: \mathrm{CH}_{4}[89: 10: 1]$, it was not expected based purely on the number of primary ions 


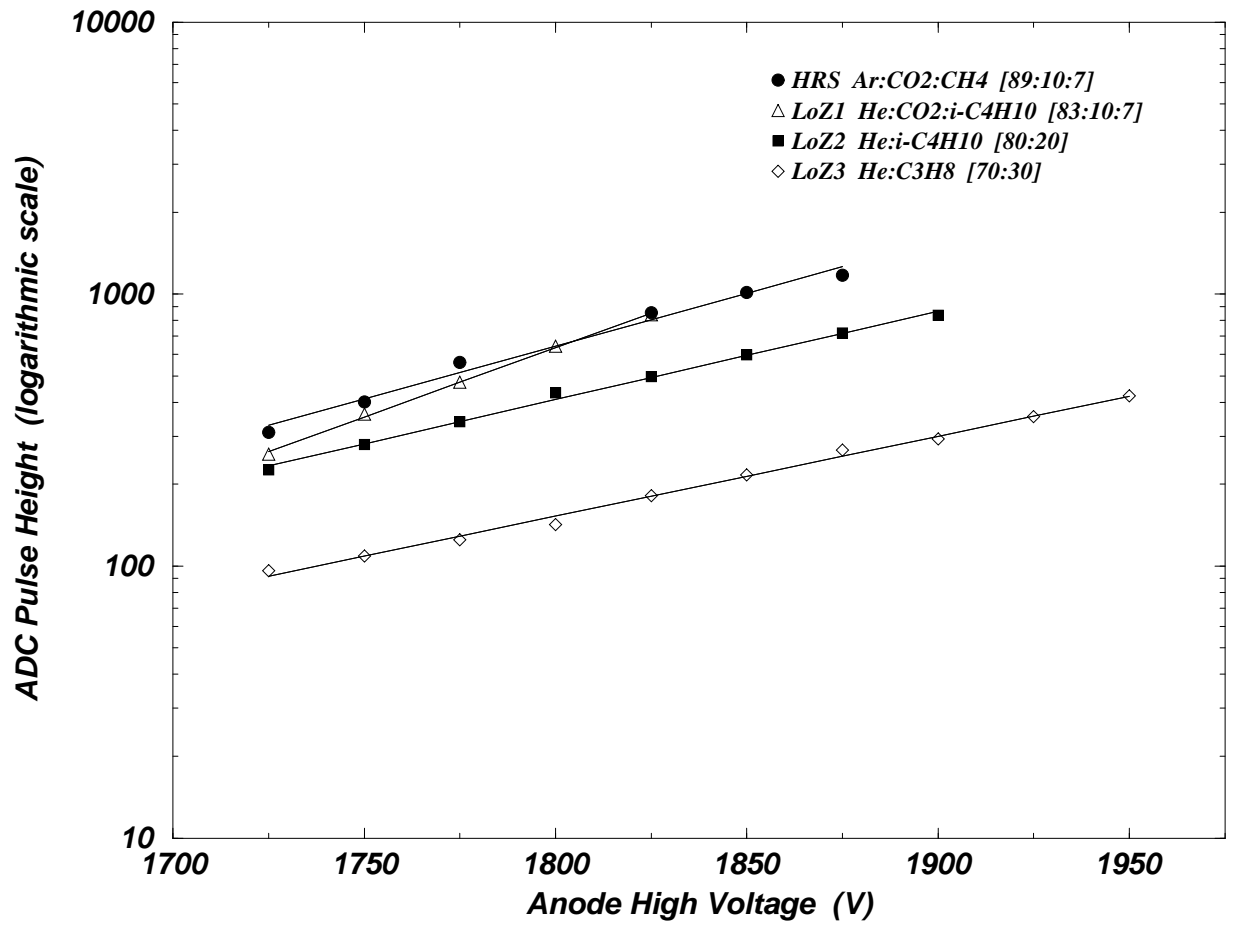

Figure 6.32: Measured mean ADC pulse height vs. anode high voltage for the four gases tested in the prototype chamber. The fit to a straight line in each case indicates that the mean pulse height has the expected exponential dependence on anode high voltage. Notice that the $\mathrm{He}: \mathrm{CO}_{2}: i-\mathrm{C}_{4} \mathrm{H}_{10}[83: 10: 7]$ and $\mathrm{He}: i-\mathrm{C}_{4} \mathrm{H}_{10}$ [80:20] gas mixtures are very similar to Ar: $\mathrm{CO}_{2}: \mathrm{CH}_{4}$ [89:10:1] in ADC pulse height, while the pulse height for $\mathrm{He}: \mathrm{C}_{3} \mathrm{H}_{8}[70: 30]$ gas is considerably lower. 
per unit track length argument.

\subsection{3 $t_{0}$ Offsets}

TDCs provide a measurement of the time between the triggering of the top scintillator paddle and charge collection on the anode. To find the time lapse between ionization and charge collection at the anode, a time offset specified as $t_{0}$ is required. The $t_{0}$ offset is the time difference between when a signal from the upper scintillator paddle is registered and the time of ionization in the chamber gas.

The wire-by-wire $t_{0}$ offsets were found after the track fitting was performed by looking at distributions of "time-like" residual distributions for each wire:

$$
\text { time-like residual }=\mid \text { measured } \text { drift distance }- \text { predicted } \text { drift distance } \mid
$$

where the measured drift distance is obtained from the TDC time values and the predicted drift distance is obtained from the fitting program. A $t_{0}$ offset shows up as a displacement of the distribution from zero.

Therefore, subtracting the $t_{0}$ offset from the converted TDC time gives the measure of time taken by the electrons to migrate from the initial ionization until they are collected at the anode. If the drift velocity of the electrons as they migrate to the sense wire is also well known, then velocity and time can be used to give information about the distance of the particle from the anode wire. This distance gives a locus of points forming a circle around the anode wire, indicating all possibilities for the point of closest approach to the wire. In the following section on segment finding and fitting, this ambiguity will be resolved and the position of the track within the chamber can be determined. 


\subsection{Segment Finding and Fitting}

Given raw data that contains information on which cells are hit for a certain event, the task of finding and fitting segments consists of looking for groups of hits that are lined up in prescribed patterns, signifying the shape of the particle track, and deciding which among the possible choices is the most likely. There are essentially three steps involved in finding and fitting segments: $(i)$ pattern recognition, $(i i)$ determining leftright ambiguities, and finally (iii) segment fitting. Thus, track fitting amounts to knowing what hit patterns to look for within the raw data, finding sets of hits that fit that description, and then taking the best fitted segments from those hits to be the true segment describing the trajectory of the particle and then combining those segments into tracks.

\subsubsection{Pattern Recognition}

Figure 5.28 in Chapter 5 shows the cell geometry of the prototype drift chamber used in this experiment. 72 anode wires were instrumented and organized into 3 "superlayers" of 4 layers each.

The segment finding algorithm begins by first breaking the cells of one of the superlayers into wedge groups of 10 cells, arranged like bowling pins. In the case of the prototype chamber, where there are 4 axial layers per axial superlayer, the wedge pattern would have the configuration shown in Figure 6.33.

Within this small subsection of the chamber's cells, all of the valid hit patterns that traverse the superlayer can be identified. At this stage, a valid pattern is one which has a hit in each of the 4 layers within a single wedge pattern. After all such 4 hit patterns have been identified, patterns with only 3 hits are then checked. The single cell at the top of the wedge acts as a pivot point, and spanning the 4 layers in each 


$$
\begin{aligned}
& \mathrm{X} \quad \text { smaller chamber radius } \\
& \begin{array}{lllllllll} 
& & & \mathrm{X} & & \mathrm{X} & & & \\
\mathrm{X} & \mathrm{X} & & \mathrm{X} & & \mathrm{X} & & \\
& & \mathrm{X} & & \mathrm{X} & & \mathrm{X} & \text { larger chamber radius }
\end{array}
\end{aligned}
$$

Figure 6.33: Cell pattern of wedge grouping used for segment recognition. The track finding program groups the cells in the chamber this way when it tests for segments.

of the 2 axial superlayers there are 8 valid hit patterns which are explicitly shown in Figure 6.34.

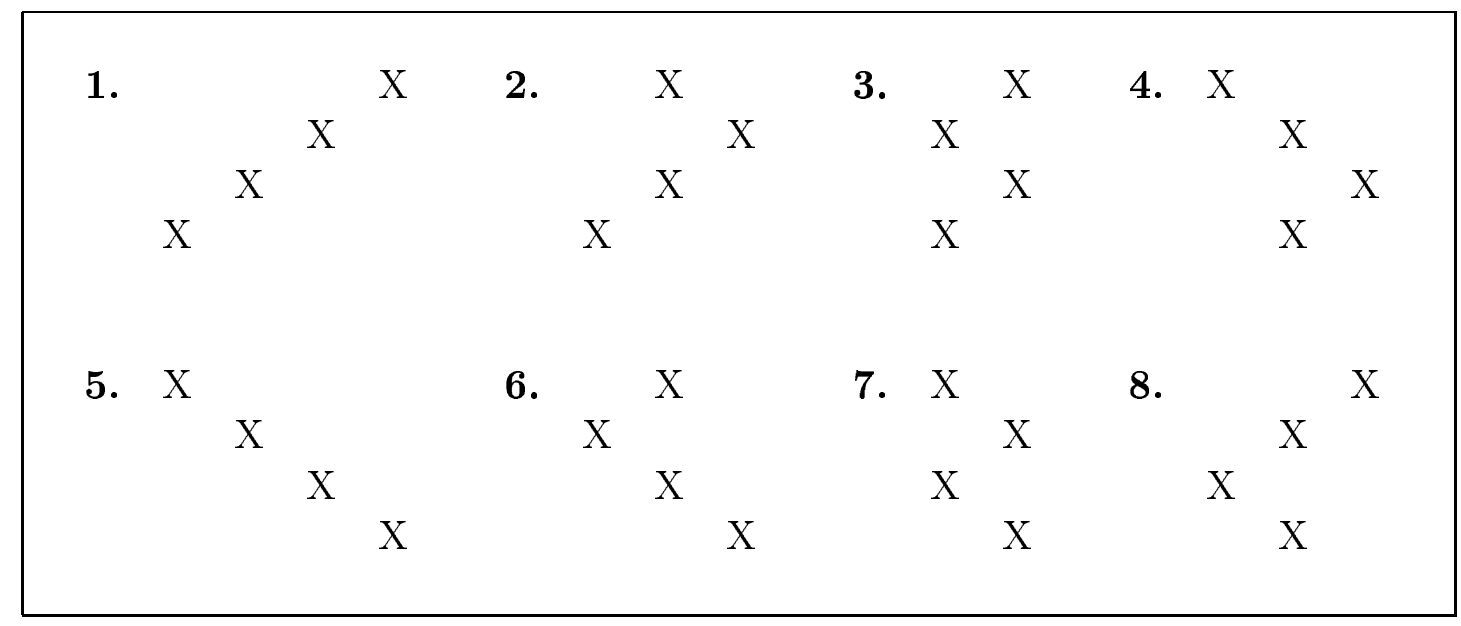

Figure 6.34: The 8 valid segment patterns that can be found within the 10 cell grouping defined in Figure 6.33. First, these patterns are searched for assuming no hits are missing from any of the layers, then later, 1 missing hit is allowed.

These cell configurations form the complete list of valid segment hit patterns, which may be compared to the raw hit data. Once a wedge of cells is isolated, the above patterns are essentially overlaid on to the observed hit pattern to see whether or not there is a match. If a match occurs, this group of 4 hits forms a segment and is then fit after left-right ambiguities have been resolved. 


\subsubsection{Left-Right Ambiguities}

At this point, whether or not a cell has been hit or not is known. The time it has taken the electrons from that hit to migrate to the sense wire using the TDC and $t_{0}$ offsets (and hence the radial distance of the track from the sense wire) is also known. However, knowing only the radial distance from the sense wire is not sufficient to constrain the trajectory of the track - the particle is free to have its point of closest approach pass through any one of the infinite number of points on the circle surrounding the anode wire because there is no way to know beforehand where the track should be (see Figure 6.35).

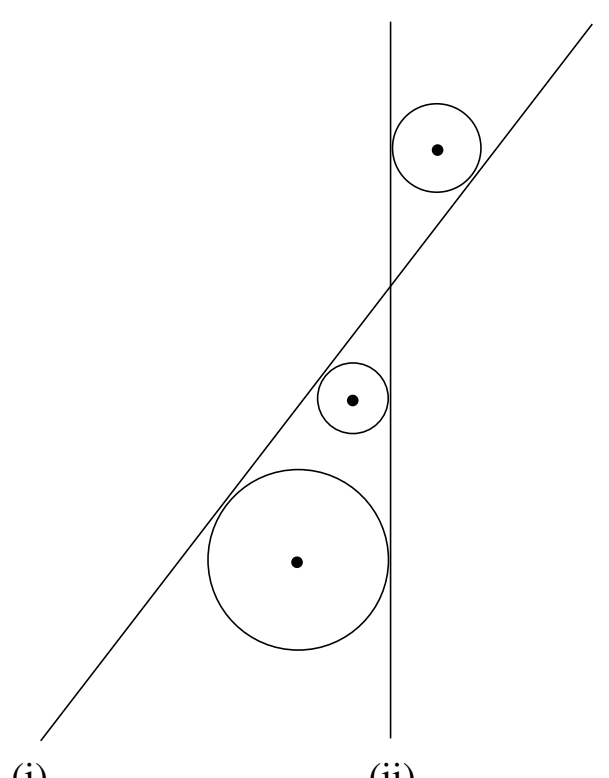

(i)

(ii)

Figure 6.35: Diagram illustrating left-right ambiguities. The time measured by the TDCs places the particle at any one of an infinite number of points on a circle surrounding the anode wire. Paths (i) and (ii) represent two possible tracks consistent with the measured TDC times for each hit. Knowing only time does not allow us to determine which side of the sense wire the track should be on. The best choice of left or right for a particular segment is determined by finding the configuration that offers the lowest $\chi^{2}$ contribution to the segment. 
In practice, the geometry of the upper and lower scintillator paddles, as well as the particular cells considered, constrain the incoming cosmic rays to be very nearly vertical. In effect, the infinite number of choices available surrounding each wire is reduced to only two, namely the left and the right, and hence the name left-right ambiguities. The segment fitting algorithm outlined below is used to resolve these ambiguities.

\subsubsection{Segment Fitting}

For each hit wire in the segment, two hypothetical paths are constructed: one in which the segment passes on the left of the wire in question and one in which it passes on the right. Both segments are fit to a straight line. After all of the allowed combinations are tried in this manner, the segment which has the best chi-square distribution ${ }^{2}, \chi^{2}$, is taken to be the true segment. Segments having a $\chi^{2} / d o f>20.0$ are discarded.

Once this stage is reached, the segment has been fit assuming only 1 hit from each of the 4 layers is present in the positions dictated by the segment recognition patterns. However, a particle may leave ionization in more than 4 cells as it passes through a superlayer, as illustrated in Figure 6.36, which means steps must be taken to consider cells adjacent to those through which the particle passed.

Beginning with the initial "seed" segment of 4 hits, additional hits that have a drift distance $10 \sigma$ away or less are tested to see whether or not they belong to this segment. As a result of this last procedure the final fitted segment that initially had only 4 hits is now complete with all of the adjacent hits that are expected to be associated with

\footnotetext{
${ }^{2}$ The $\chi^{2}$ is a goodness-of-fit distribution which is defined as [24]:

$$
\chi^{2} \equiv \sum_{i=1}^{n}\left\{\frac{1}{\sigma_{i}^{2}}\left[y_{i}-y\left(x_{i}\right)\right]^{2}\right\} \text {. }
$$
}




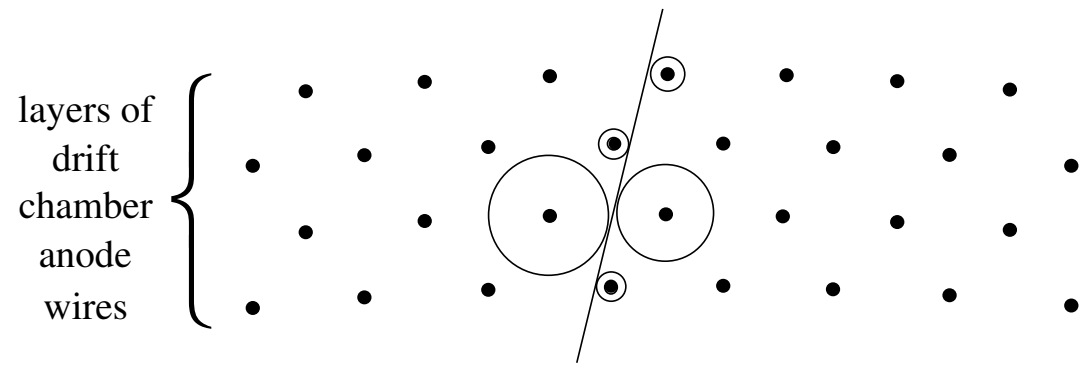

Figure 6.36: Shown is an example of how a segment formed with hits from 4 layers can have more than 4 hits associated with it.

it and that have also passed all of the previously described cut criteria.

Having exhausted all of the possibilities of constructing a seed segment with 1 hit in each of the four layers, the next step is to allow for 1 missing hit in the group of four that the segment recognition algorithm expects. The algorithm for this procedure follows in a manner similar to that outlined above. In effect, we have placed a cut on the data: for a given group, a hit is necessary in at least three of the four layers before the hit arrangement is considered for possible segments.

After completing this analysis on one group of cells, the pinnacle of the wedge is moved over one cell and the process is repeated until the first axial superlayer has been spanned, at which point we continue on to the second axial superlayer until all possible cell groups have been considered and all possible segments have been found.

\subsection{Track Fitting}

In two dimensions, for a charged particle in a chamber with no applied magnetic field, each segment is parameterized by two track parameters $d_{0}$ and $\phi_{0}$ as defined in Figure 6.37.

Figure 6.37 shows an end-view in the $x-y$ plane looking along the $z$ axis of the 


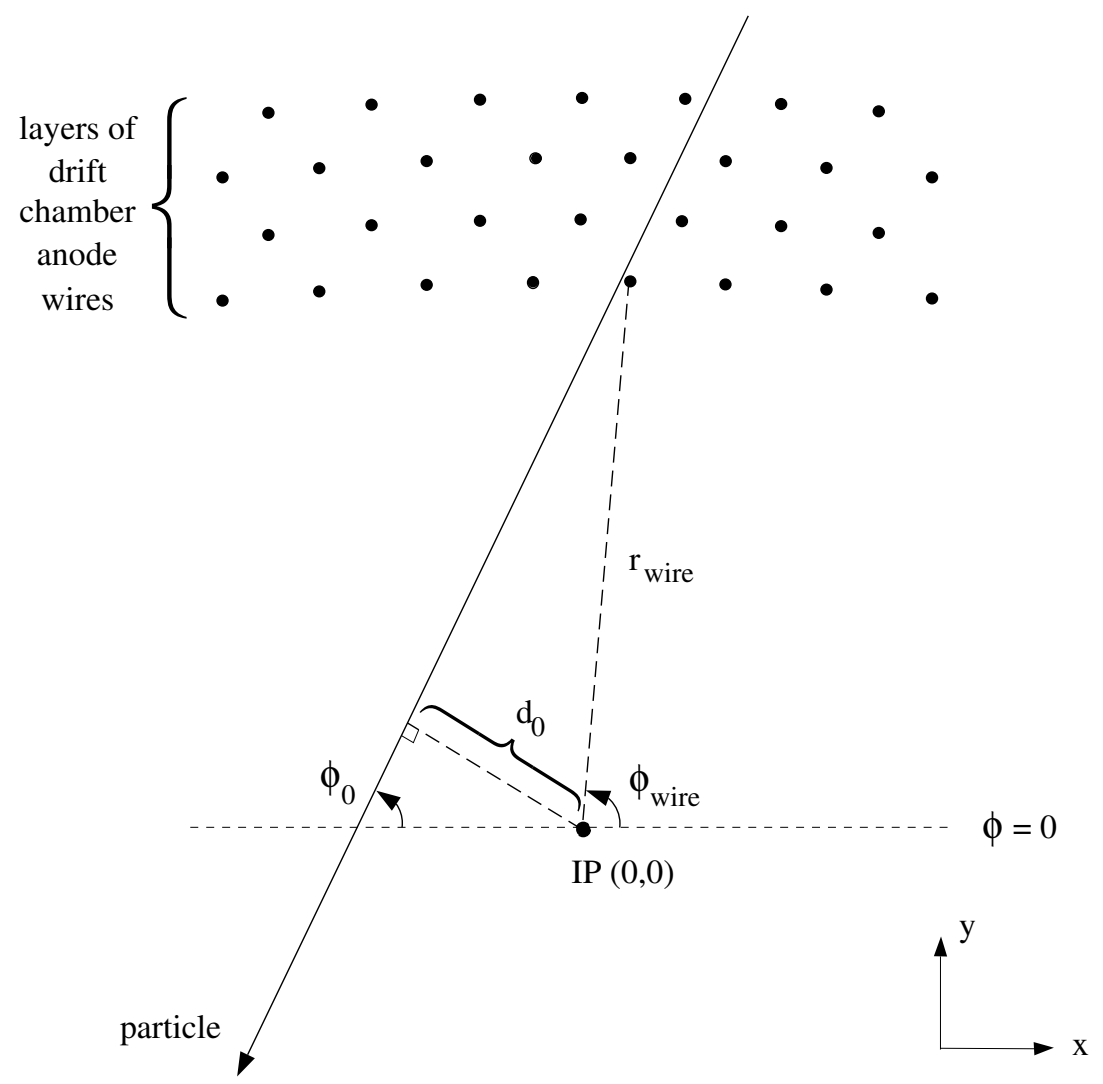

Figure 6.37: Diagram illustrating the definition of the track parameters $d_{0}$ and $\phi_{0}$. In a drift chamber with no applied magnetic field, these two parameters define the trajectory of the particle in the $x-y$ plane.

prototype drift chamber illustrated with only 4 layers of wires rather than 12 . The fit parameter $d_{0}$ is defined as the signed impact parameter or distance of closest approach to the origin of the coordinate system called the interaction point (IP). The second fit parameter, $\phi_{0}$, is the angle that the track makes with the $\phi=0$ line.

Taking all hits that comprise the two segments, a least-squares fit to the hypothesis of a straight line is then performed. The fit is accomplished by assuming that the equation involving the track parameters is linear; because of this approximation, the 
fit may be iterated. For a given iteration, the change in the fitted parameters, $\alpha$ :

$$
\alpha=\left(\begin{array}{c}
d_{0} \\
\phi_{0}
\end{array}\right)
$$

is given by [25]:

$$
\eta=V_{A} A^{T} V_{y}^{-1} \Delta y
$$

where $\eta$ is the change in track parameters $\alpha$ such that $\alpha^{\prime}=\alpha+\eta . V_{y}^{-1}$ is the inverse of the measured drift distance covariance ${ }^{3}$ matrix, and is often referred to as the "weight matrix" as it is used to weight the errors in the fit. Written explicitly, it is simply a diagonal matrix including the errors weighted by drift distance, $\sigma_{n}$, associated with the $n$ independently measured values of the drift distance (that is, for $n$ hits):

$$
V_{y}^{-1}=\left(\begin{array}{ccc}
1 / \sigma_{1}^{2} & 0 & 0 \\
0 & 1 / \sigma_{2}^{2} & 0 \\
\vdots & \vdots & \vdots \\
0 & 0 & 1 / \sigma_{n}^{2}
\end{array}\right)
$$

$A^{T}$ is the transpose of the matrix $A$ which contains the derivatives of the calculated drift distance with respect to the two track parameters:

$$
A=\left(\begin{array}{cc}
\frac{\partial d_{\text {drift }_{1}}}{\partial \phi_{0}} & \frac{\partial d_{\text {drift }_{1}}}{\partial d_{0}} \\
\frac{\partial d_{d r i f t_{2}}}{\partial \phi_{0}} & \frac{\partial d_{d r i f t_{2}}}{\partial d_{0}} \\
\vdots & \vdots \\
\frac{\partial d_{d r i f t_{n}}}{\partial \phi_{0}} & \frac{\partial d_{d r i f t_{n}}}{\partial d_{0}}
\end{array}\right)
$$

where [26]

$$
\frac{\partial d_{d r i f t}}{\partial d_{0}}=1, \quad \frac{\partial d_{d r i f t}}{\partial \phi_{0}}=r_{w i r e} \cos \left(\phi_{\text {wire }}-\phi_{0}\right) .
$$

\footnotetext{
${ }^{3}$ The covariance matrix contains information about the errors of parameters as well as on how these parameters are correlated (i.e., on how a change in one parameter affects another).
} 
In Equation 6.119, $V_{A}=\left(A^{T} V_{y}^{-1} A\right)^{-1}$ is the symmetric covariance (error) matrix of the fitted track parameters

$$
V_{A}=\left(\begin{array}{ll}
v_{11} & v_{12} \\
v_{12} & v_{22}
\end{array}\right),
$$

and $\Delta \mathrm{y}$ is the residual which is defined to be

$$
\text { residual }=d_{d r i f t}(\text { measured })-d_{d r i f t}(\text { predicted })
$$

where the measured drift distance is found using the drift time (obtained using the converted TDC time value and $t_{0}$ offsets) and drift velocity, and the predicted drift distance is that estimated by the fitting program. For $n$ values of the drift distance (that is, for $n$ hits) and 2 track parameters, the drift distance covariance matrix $V_{y}$ has dimensions $(n \times n)$, which means that the track parameter covariance matrix $V_{A}$ has dimensions $(2 \times 2)$.

The fit is said to converge if the change in $\chi^{2}$ from one iteration to the next is less than 0.05. After the fitting has converged, if the resulting track has a $\chi^{2}>20.0 /$ dof it is dropped from consideration.

Using the following expressions to describe the coordinates of the hit, $x_{0}$ and $y_{0}$ :

$$
\begin{gathered}
x_{0}=-d_{0} \sin \left(\phi_{0}\right)-r_{\text {wire }} \cos \left(\phi_{\text {wire }}\right), \\
y_{0}=d_{0} \cos \left(\phi_{0}\right)-r_{\text {wire }} \sin \left(\phi_{\text {wire }}\right)
\end{gathered}
$$

where $d_{0}$ and $\phi_{0}$ are the track fit parameters, and $r_{\text {wire }}, \phi_{\text {wire }}$ are the coordinates of the sense wire at the centre of the hit cell (see Figure 6.37), the expected drift distance can be determined. The distance from the particle track to the sense wire as predicted from the fitting algorithm can be found using:

$$
\text { predicted drift distance }=y_{0} \cos \left(\phi_{0}\right)-x_{0} \sin \left(\phi_{0}\right) \text {. }
$$




\subsection{Track Refitting}

It may turn out that in the track fitting described above, a few hits are at the limit of the $\sigma$ and $\chi^{2}$ cuts imposed, and in the fitting process these hits would tend to pull the resulting fit of all the hits in their direction. If a track is skewed toward a given hit, this would mean that the predicted drift distance obtained through that fit is biased, and consequently the residual value would also be biased. If, instead, this hit is not included while fitting, then a less biased value for the residual of that particular hit will be obtained. Here, new residuals are obtained from the final fitted track by dropping hits one at a time from the track and refitting the hits that remain to get the residual contribution of the dropped hit. However, the residual obtained when hits are left out of the fit has a contribution due to the intrinsic resolution of the wire and another due to the error on the projection of the track into the cell. The track error is an estimate of the error in the residual caused by this track projection, and is calculated for each chamber layer using the error from a straight line fit and the assumed spatial resolutions for the other hits in the track:

$$
t_{\text {error }}^{2}=v_{22}\left(\frac{\partial d_{d r i f t}}{\partial d_{0}}\right)^{2}+v_{11}\left(\frac{\partial d_{d r i f t}}{\partial \phi_{0}}\right)^{2}+2 v_{12}\left(\frac{\partial d_{d r i f t}}{\partial d_{0}}\right)\left(\frac{\partial d_{d r i f t}}{\partial \phi_{0}}\right)
$$

The error matrix elements $v_{11}, v_{22}$, and $v_{12}$ come from Equation 6.123 and indicate the errors in the fitted track parameters. The partial derivatives are explicitly given in Equation 6.122. Because the track error depends on resolution values found only after some analysis, the determination of the error is an iterative process.

\subsection{Drift Functions}

Finding drift functions, or the time-distance relations of the drift electrons, is an iterative process which necessarily means that track finding is also an iterative process. 
Because of the numerous environmental factors involved (for example, temperature and pressure), a theoretical drift function will not globally apply to the data. Instead, an experimental approach for finding the time-distance relationship must be pursued.

For each layer in the chamber, all of the hits in a given layer for a particular gas run are considered, where each hit has an associated drift time (from the TDC) and a predicted drift distance (from the track refitting part of the fitting program). The entire time interval over which hits arrive at the sense wire is divided into 30 bins, or time windows, for which the drift distances within each individual time bin are described by a single Gaussian distribution, unless the drift distances are close to the sense wire. In the former case, the distribution of drift distances is fit to a single Gaussian distribution excluding regions $2.5 \sigma$ on either side of the mean, and the mean of the distribution is taken to be the best estimate of the drift distance for that time bin. In the latter case, which applies to the first 5 time bins, the distribution is fit to 2 Gaussian distributions and the mean of the peak is taken as the best estimate of the drift distance.

Once this has been done this for all time bins in the time interval, the drift velocity of the gas mixture has been determined, as shown in Figure 6.38. These new estimates of the time-distance relationship are then used in the next iteration of the track finding program. Depending on the initial estimate for the time-distance relation, it usually takes 4 or 5 iterations before the process converges to a point where the resolution (see Chapter 8) is constant within the associated statistical error. 


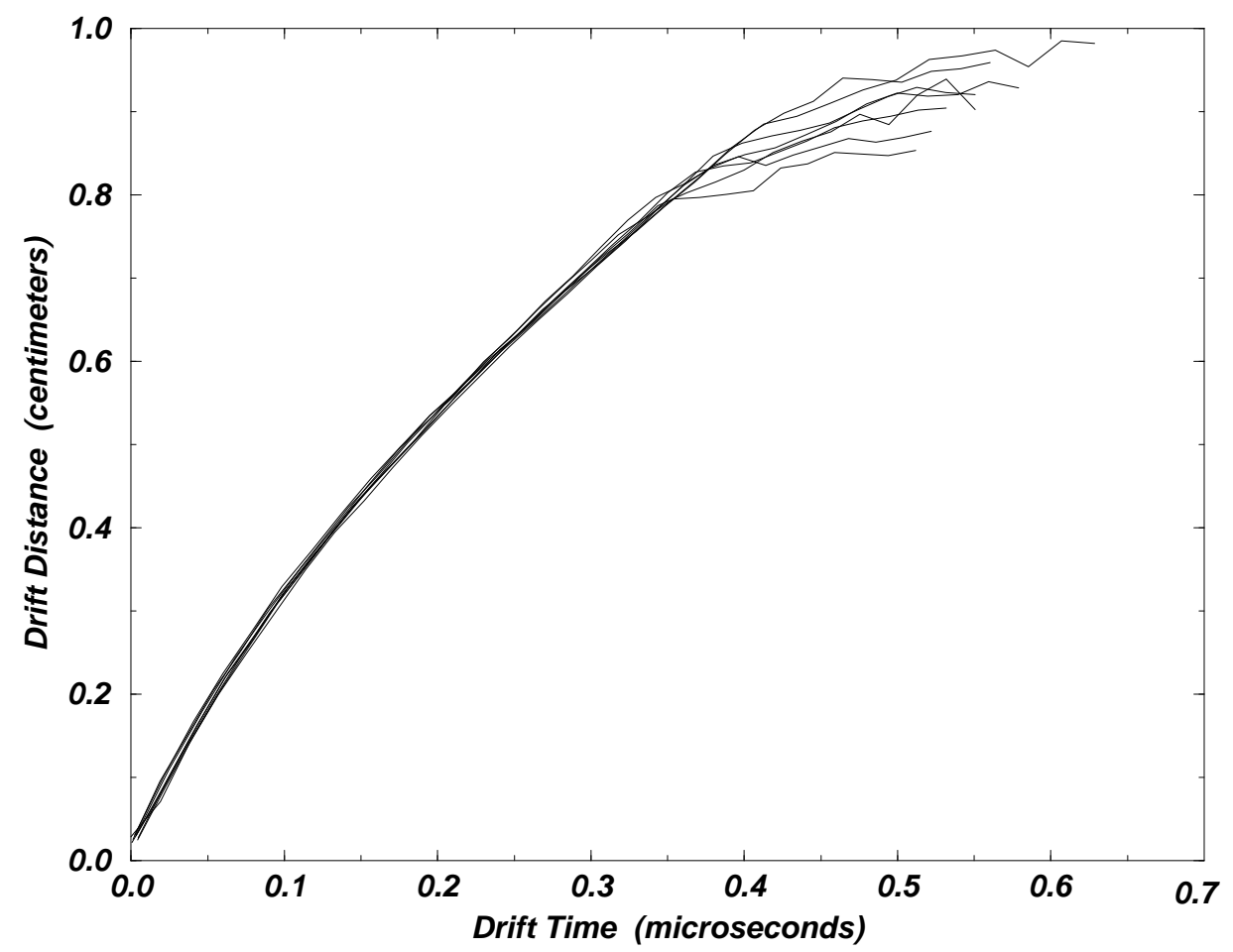

Figure 6.38: Drift Function for $\mathrm{He}: i-\mathrm{C}_{4} \mathrm{H}_{10}$ [80:20] gas at anode voltage $1850 \mathrm{~V}$. The 8 lines indicate the time-distance relation for each of the 8 axial layers used in the prototype chamber. Notice that over a large region, the drift function is approximately constant; however, near the edge of the cell boundary $(0.8-1.0 \mathrm{~cm})$ the magnetic field is not as uniform as it is over the interior region, which is reflected in the non-linear shape of the drift velocity curve at those distances. A time cut is applied so that these outlying, extremely non-linear contributions are excluded. 


\section{Chapter 7}

\section{Efficiency Studies}

\subsection{Introduction}

In this chapter, chamber efficiency as a function of gas mixture will be investigated. Using the fit from the final tracks found in Chapter 6 for each of the four chamber gases, chamber efficiency will be tested by considering cells which should register a hit in a given event, but do not. Factors that are expected to contribute to inefficiency include having tracks pass too close to the sense wire giving large deviations from the time-distance relation and poor electric field shaping at the cell boundaries.

\subsection{Pseudo-efficiency}

Before a more rigorous efficiency algorithm was implemented, a quick check on efficiency was performed using a quantity called pseudo-efficiency, which is simply the ratio of the number of tracks found in a particular gas run to the number of events in that run:

$$
\text { pseudo-efficiency }=\frac{\text { Number of Tracks }}{\text { Number of Events }}
$$

An "event" is recorded when both the upper and lower scintillator paddles detect a cosmic ray passing through them, while a "track" found in an event has passed all of the hit requirements, $\chi^{2}$ and distance cuts discussed in Chapter 6. Typically, the pseudoefficiency is approximately $50 \%$ for a gas operating in its optimal high voltage region. The results acquired by looking at pseudo-efficiency are summarized in Table 7.4 along 
with the results of a more detailed efficiency study. The trends observed with the more thorough efficiency study are very similar to those obtained through these simple means of counting events and tracks. Hence, pseudo-efficiency is a useful cross check for the efficiency studies described in the following section, but will not be used for any quantitative comparisons.

\subsection{Efficiency Algorithm}

The track parameters $d_{0}$ and $\phi_{0}$ for the final fitted tracks are easily manipulated to form the equation of a line, $y=m x+b$ :

$$
y=\left(x-x_{0}\right) \tan \left(\phi_{0}\right)+y_{0}
$$

where $m=\tan \left(\phi_{0}\right)$ is the slope of the track, $b=y_{0}-x_{0} \tan \left(\phi_{0}\right)$ is the $\mathrm{y}$-intercept, and where the Cartesian coordinates $x_{0}$ and $y_{0}$ representing the distance of closest approach that the track makes with the IP are given by the equations:

$$
x_{0}=-d_{0} \sin \left(\phi_{0}\right), \quad y_{0}=d_{0} \sin \left(\phi_{0}\right)
$$

Considering all of the wires in both axial superlayers, the distance of closest approach that the track makes with each wire is calculated. The fact that every wire in the chamber is considered allows for the possibility for there to be more than one valid hit for a given layer.

To find the distance, $D^{\prime}$, the distance of closest approach between a line and a point, consider the distance, $D$, between an arbitrary point on the track and a sense wire within the chamber:

$$
D=\sqrt{\left(x-x_{w i r e}\right)^{2}+\left(y-y_{w i r e}\right)^{2}}
$$

where $x_{w i r e}$ and $y_{\text {wire }}$ are the Cartesian coordinates of the sense wire under consideration. The distance between the track and the sense wire is minimized in the standard 
manner by setting the derivative of $D$ with respect to one of the track coordinates to zero. Choosing to minimize the above distance with respect to $x$, this analysis yields the following expression for $D^{\prime}$, the distance of closest approach:

$$
D^{\prime}=\sqrt{\left(x_{\text {min }}-x_{w i r e}\right)^{2}+\left(m x_{\text {min }}+b-y_{w i r e}\right)^{2}}
$$

where

$$
x_{\text {min }}=\frac{m\left(y_{w i r e}-b\right)+x_{w i r e}}{1+m^{2}} .
$$

In order to decide whether or not the track has passed through a particular cell, a circle is placed around each sense wire as an approximation of the cell area. If a track intersects this circle, then a hit is expected in that cell. Due to the slightly different geometry, the hexagonal and pentagonal cells will be considered separately.

The hexagonal cells are approximated by a circle inscribed within a hexagon which includes $91 \%$ of the cell area - it was not felt that the effort needed to exactly model the cell was necessary, as the electric field in the corners of the hexagon tends to be irregular and has large derivatives. Whereas, with the pentagonal cells, more care must be taken before the circular approximation can be applied. However, as was argued in Chapter 6 for the left-right track ambiguities, tracks that are very nearly vertical will pass only on the right- or left-hand side of the sense wire rather than above or below the wire or through any other region in the cell. Therefore, as the sides of the pentagonal cells are no different from those of the hexagonal cells, the same circular approximation is valid for the pentagonal cells.

The track and a circle surrounding each sense wire within the detector space are now well specified, so all that remains is to test whether or not the distance of closest approach is within the cell radius. If it is, a hit is expected and the list of observed hits that make up the track under consideration is checked to see if indeed a hit was detected. An efficient chamber will detect all such expected hits; if a hit is expected 
but the chamber failed to identify it, this is a measure of inefficiency. If, on the other hand, the circle and track do not intersect, then a hit is not expected in the cell, and the algorithm moves on to the next wire where the above procedure is repeated.

Once all of the tracks for a particular gas run have been analyzed, the total number of times a hit was expected and seen by the chamber is known as well as the total number of times a hit was expected but not detected by the chamber. Prototype efficiency is then defined as:

$$
\text { efficiency }=\frac{\text { expect \& hit }}{(\text { expect \& hit })+(\text { expect \& miss })}=\frac{K}{N}
$$

where the denominator $(N)$ is simply the normalization factor which indicates the total number of times a hit was expected within the chamber which is constant for a given gas run.

The error in efficiency is governed by the binomial distribution since there are only two possibilities allowed: either a hit is expected and one is detected, or a hit is expected and one is not detected. The variance for a binomial distribution is given by:

$$
\sigma^{2}=N p(1-p)
$$

where $N$ is the total number of trials (the normalization factor in Equation 7.135) and $p$ is the efficiency, or the probability of "success". The error in $p$ is determined using the error in $K$, namely:

$$
\sigma_{K}=\sqrt{N p(1-p)}
$$

Simple propagation of errors gives the corresponding error in $p$ as:

$$
p=\frac{K}{N} \quad \rightarrow \quad \sigma_{p}=\frac{\sigma_{K}}{N}=\frac{\sqrt{N p(1-p)}}{N} .
$$

Substituting for $p$ in Equation 7.138, an expression for the error in efficiency $(p)$ is obtained in terms of the number of times a hit is expected and detected $(K)$ and the 
total number times a hit was expected $(N)$ :

$$
\sigma_{e f f i c i e n c y}=\sqrt{\frac{K}{N^{3}}(N-K)} .
$$

\subsection{Analysis Data Reduction}

In Chapter 6, the various data cuts employed during the track fitting and finding procedure were discussed. For both the efficiency studies and the resolution studies that will be outlined in Chapter 8 , the data were further reduced by the introduction of two additional constraints.

\subsubsection{Entrance Angle}

The first cut involves placing an upper boundary of approximately $\pm 17^{\circ}$ on the entrance angle as measured from the radial direction that is allowed for the incoming particle. The analysis data will then consist of events in which the tracks are more vertical than those that simply meet the geometry requirements set by the scintillator paddles as illustrated in Figure 7.39 (a diagram showing the scintillator paddles is shown in Figure 5.29). The more vertical a track is, the more valid the left-right approximation will be when fitting segments.

\subsubsection{Wires Used}

The second cut that was applied limited the wires used in the analysis to be only those in the very centre of the prototype in an attempt to simulate a symmetric drift chamber (see Figure 7.40).

The full scale drift chamber that will eventually be built for the $B_{A} B_{A R}$ detector will be a complete cylinder where no cells will be at an edge in $\phi$ - in other words, the drift chamber cells in the $x-y$ plane will form a complete circle. The prototype being used 


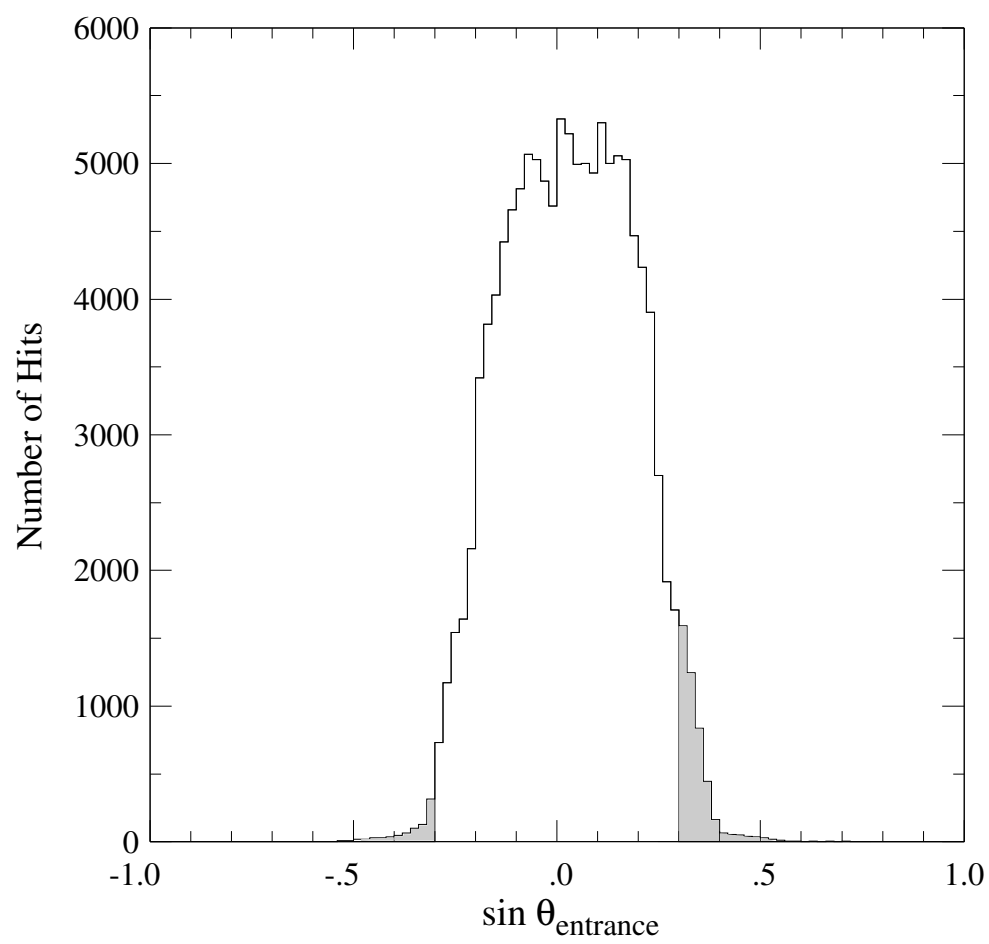

Figure 7.39: Histogram of the sine of the entrance angle for each hit showing the effect of the cut used in the analysis. This particular histogram was obtained using $\mathrm{He}: i-\mathrm{C}_{4} \mathrm{H}_{10}[80: 20]$ gas at $1850 \mathrm{~V}$. The shaded areas represent hits that have entrance angles greater than approximately $\pm 17^{\circ}\left(-0.3>\sin \theta_{\text {entrance }}>0.3\right)$.

in these studies, however, represents only a small wedge of that cylinder, which means that the cells located at the edges will experience non-uniformities that will not be present in the full scale chamber. Therefore, in order to minimize these "edge-effects", the analysis was performed using only the central wires of the prototype which will have the most uniform electric fields. 


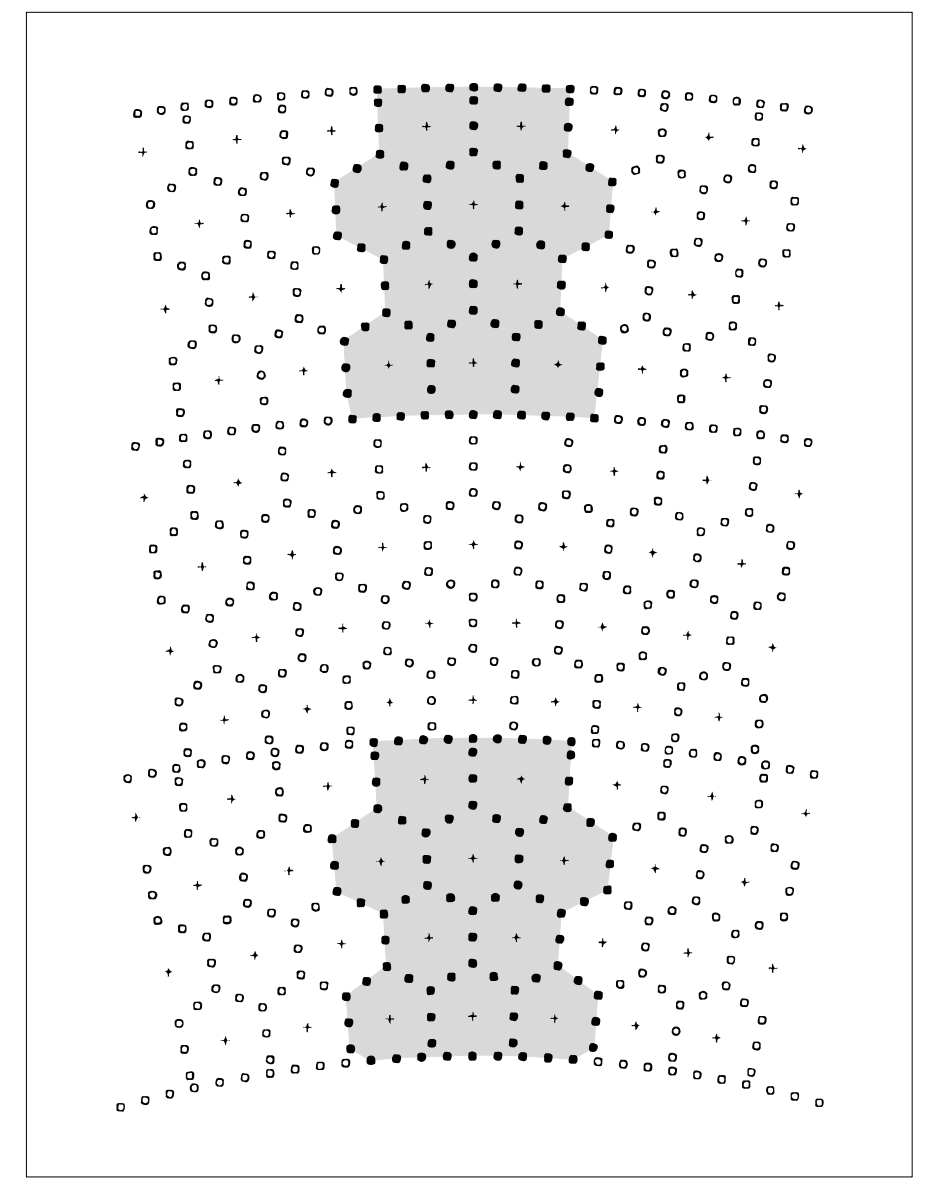

Figure 7.40: Diagram showing the prototype wires used in the analysis. Only the central wires were used in order to simulate a cylindrically symmetric drift chamber.

\subsection{Efficiency Results}

It was expected that the number of tracks for a given gas run should sample the cell space uniformly ${ }^{1}$, so as a zeroth order check on the efficiency algorithm, the number of times a track was seen at a given radial distance of closest approach was plotted.

\footnotetext{
${ }^{1}$ Although the distance of closest approach is a radial quantity, once the cell area is binned, the tracks do not uniformly populate the annuli surrounding the anode, but rather the left- and right-most portions because the tracks are approximately vertical.
} 


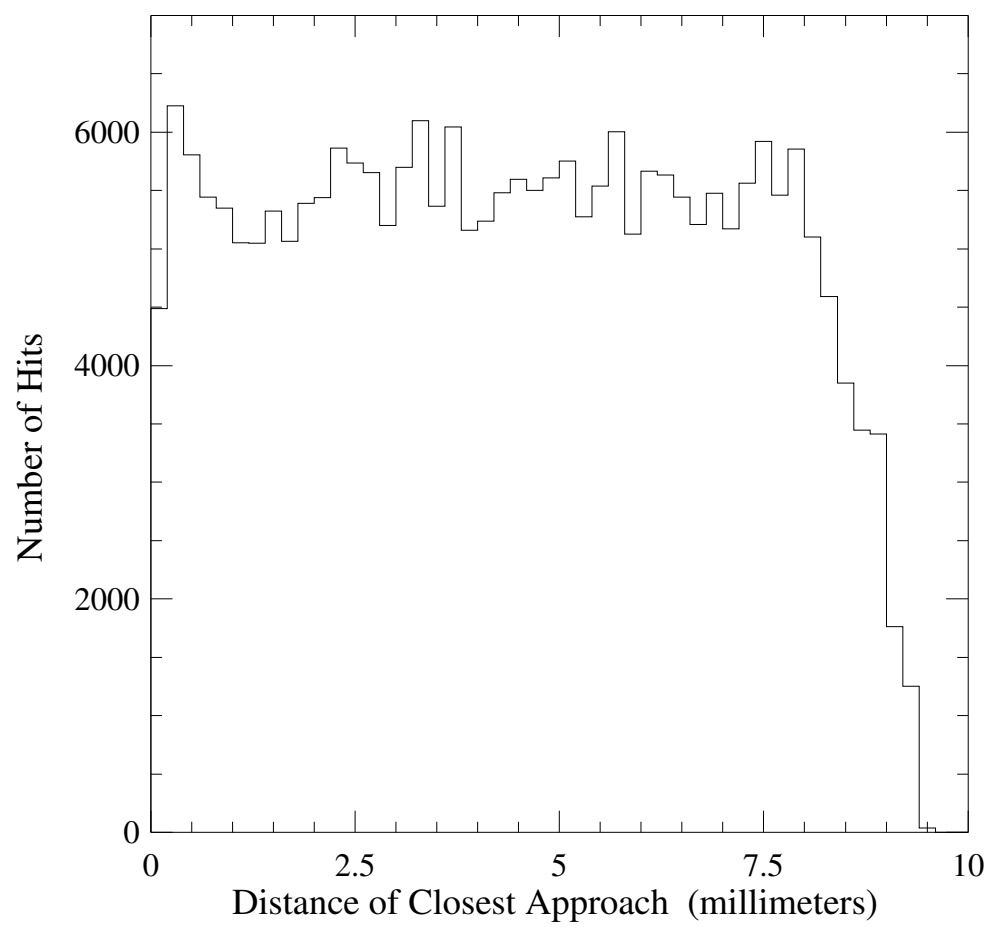

Figure 7.41: Histogram of the distance of closest approach per hit for $\mathrm{He}: i-\mathrm{C}_{4} \mathrm{H}_{10}$ [80:20] gas at $1850 \mathrm{~V}$. Notice that the distribution is approximately constant throughout the cell except at high distances corresponding to the edge of the cell.

The distribution of distances of closest approach shown in Figure 7.41 is approximately constant throughout the cell which lends credibility to the efficiency algorithm. Regions that have a very large distance of closest approach have fewer counts in them because not all cells have a radius that large (remember that the radial size of cells in the prototype chamber increases as layer number increases). Also, there is a slight deficit near the sense wire because tracks that pass too close will not have had time to initiate an electron avalanche and therefore will not be detected.

One of the first questions that needs to be answered about each gas is where the optimal high voltage operating region is. Different gas mixtures will have different 
efficiencies depending on what high voltage is used; the results of this study are summarized in Table 7.4. Notice how the trends in the pseudo-efficiency match those of the more sophisticated efficiency calculation. The absolute pseudo-efficiency numbers, however, are not very meaningful.

Figure 7.42 shows a plot of efficiency as a function of applied anode high voltage which indicates the regions where efficiency is optimum.

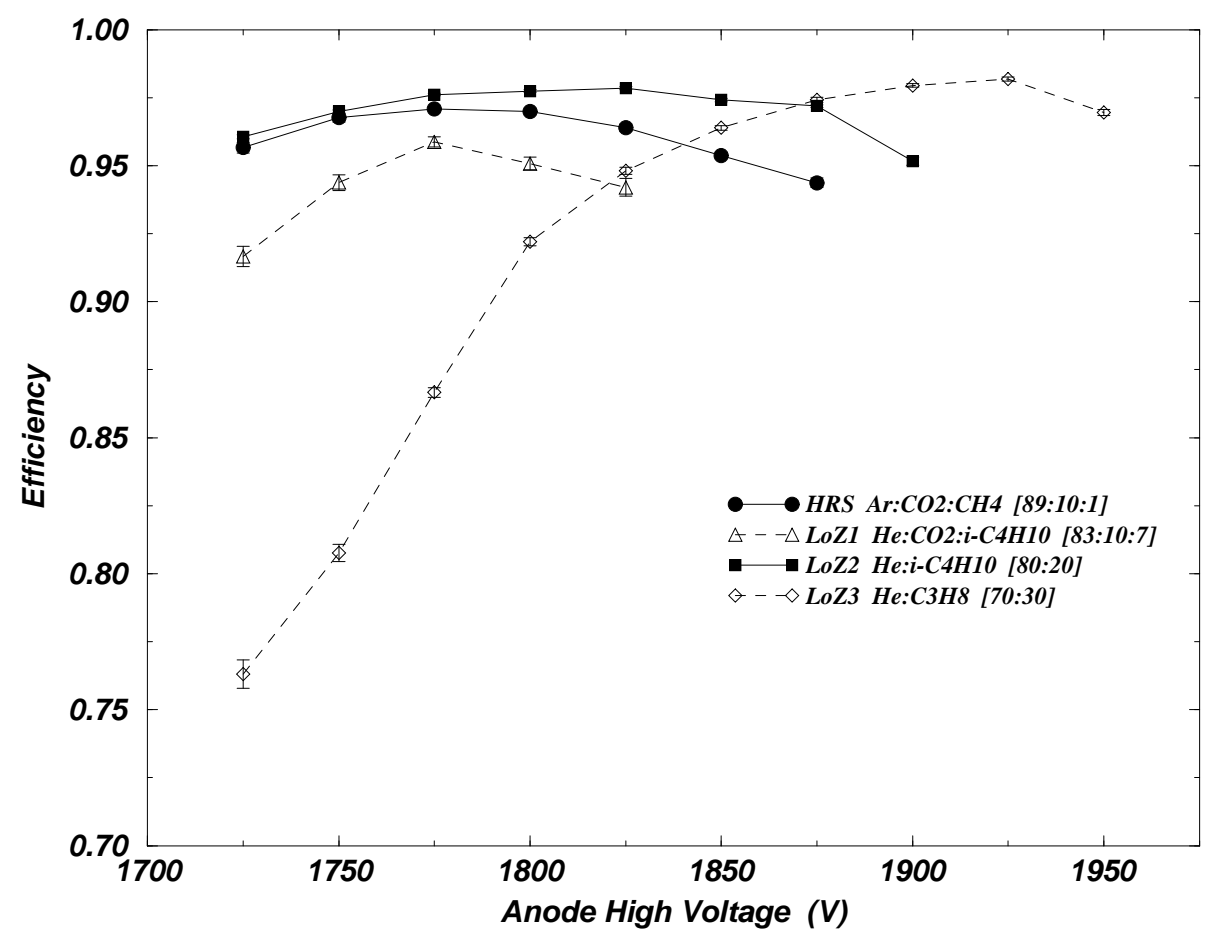

Figure 7.42: Efficiency vs. anode high voltage for all gases tested in the prototype chamber. Notice that all gases have efficiencies greater than 0.95 once the optimal high voltage operating regions are found. Also notice that $\mathrm{He}: \mathrm{C}_{3} \mathrm{H}_{8}[70: 30]$ gas starts out with the poorest efficiency, but attains the best efficiencies of all gas mixtures tested at higher voltages. 


\begin{tabular}{|c|c|c|c|}
\hline Gas Mixture & $\begin{array}{c}\text { Anode } \\
\text { High Voltage } \\
\text { (V) }\end{array}$ & $\begin{array}{c}\text { Pseudo- } \\
\text { Efficiency }\end{array}$ & $\begin{array}{c}\text { Average } \\
\text { Efficiency }\end{array}$ \\
\hline Ar: $\mathrm{CO}_{2}: \mathrm{CH}_{4}[89: 10: 1]$ & $\begin{array}{l}1725 \\
1750 \\
1775 \\
1800 \\
1825 \\
1850 \\
1875 \\
\end{array}$ & $\begin{array}{l}0.437 \\
0.479 \\
0.467 \\
0.465 \\
0.419 \\
0.346 \\
0.236 \\
\end{array}$ & $\begin{array}{l}0.9566 \pm 0.0019 \\
0.9676 \pm 0.0016 \\
0.9708 \pm 0.0007 \\
0.9699 \pm 0.0006 \\
0.9640 \pm 0.0013 \\
0.9537 \pm 0.0011 \\
0.9437 \pm 0.0018\end{array}$ \\
\hline $\mathrm{He}: \mathrm{CO}_{2}: i-\mathrm{C}_{4} \mathrm{H}_{10}[83: 10: 7]$ & $\begin{array}{l}1725 \\
1750 \\
1775 \\
1800 \\
1825\end{array}$ & $\begin{array}{l}0.385 \\
0.444 \\
0.490 \\
0.451 \\
0.312\end{array}$ & $\begin{array}{l}0.9166 \pm 0.0037 \\
0.9438 \pm 0.0029 \\
0.9587 \pm 0.0019 \\
0.9508 \pm 0.0023 \\
0.9420 \pm 0.0033\end{array}$ \\
\hline$\overline{\mathrm{He}}: i-\mathrm{C}_{4} \mathrm{H}_{10}[80: 20]$ & $\begin{array}{l}1725 \\
1750 \\
1775 \\
1800 \\
1825 \\
1850 \\
1875 \\
1900\end{array}$ & $\begin{array}{l}0.388 \\
0.509 \\
0.530 \\
0.523 \\
0.448 \\
0.485 \\
0.465 \\
0.323\end{array}$ & $\begin{array}{l}0.9606 \pm 0.0007 \\
0.9699 \pm 0.0006 \\
0.9761 \pm 0.0005 \\
0.9774 \pm 0.0005 \\
0.9785 \pm 0.0005 \\
0.9741 \pm 0.0006 \\
0.9719 \pm 0.0005 \\
0.9517 \pm 0.0015\end{array}$ \\
\hline$\overline{\mathrm{He}}: \mathrm{C}_{3} \mathrm{H}_{8}[70: 30]$ & $\begin{array}{l}1725 \\
1750 \\
1775 \\
1800 \\
1825 \\
1850 \\
1875 \\
1900 \\
1925 \\
1950\end{array}$ & $\begin{array}{l}0.061 \\
0.145 \\
0.268 \\
0.381 \\
0.440 \\
0.511 \\
0.530 \\
0.555 \\
0.541 \\
0.516\end{array}$ & $\begin{array}{l}0.7630 \pm 0.0052 \\
0.8076 \pm 0.0032 \\
0.8666 \pm 0.0018 \\
0.9221 \pm 0.0015 \\
0.9481 \pm 0.0013 \\
0.9639 \pm 0.0008 \\
0.9742 \pm 0.0009 \\
0.9795 \pm 0.0006 \\
0.9818 \pm 0.0008 \\
0.9696 \pm 0.0011\end{array}$ \\
\hline
\end{tabular}

Table 7.4: Table of results indicating pseudo-efficiency and average efficiency for the four chamber gases at various operating voltages. Refer to the text for definitions of these two efficiencies. 
All gas mixtures have efficiencies greater than 0.95 once the optimal high voltage operating regions are found. Notice that $\mathrm{He}: \mathrm{C}_{3} \mathrm{H}_{8}$ [70:30] gas starts out with the poorest efficiency, but attains the best efficiencies of all at higher voltages.

A second investigation studies how efficiency varies with location within the cell. Efficiency is expected to be poor close to the sense wire (at small radii) since, as was mentioned above, tracks that pass too close to the wire will not be detected due to the fact that ionized electrons will not have had enough time to start a sufficiently large number of subsequent ionizations which would then lead to an electron avalanche, and therefore insufficient charge will amass at the sense wire and the TDCs will not trigger indicating an event. In this way, a track could well pass near to the sense wire without the chamber signifying its presence, contributing to inefficiency. At large distances from the sense wire (large radii), the electric field is not as uniform as it is in the interior of the cell, which means that charge collection at these large radii is comparatively not as efficient. This in turn translates into a cell inefficiency. Shown in Figure 7.43 is the dependence of efficiency on drift distance.

As expected, efficiency is slightly poorer near the sense wire as well as near the cell boundary where the error is higher due to lower statistics (fewer tracks that should have passed through the cell). The interior of the cell from 2-8 $\mathrm{mm}$ has fairly constant, high efficiency.

One concern might be that there are systematic errors affecting the results. Perhaps one layer within the chamber consistently provides markedly poorer efficiency for some reason, or a few wires may have poor performance.

Consider first the efficiency for each wire as shown in Figure 7.44. The blank region in the middle of the plot indicates the stereo layer of wires which were not used in the analysis. The remaining gaps are due to wires that are either not instrumented or are not being considered (recall that only the wires in the very central regions of the 


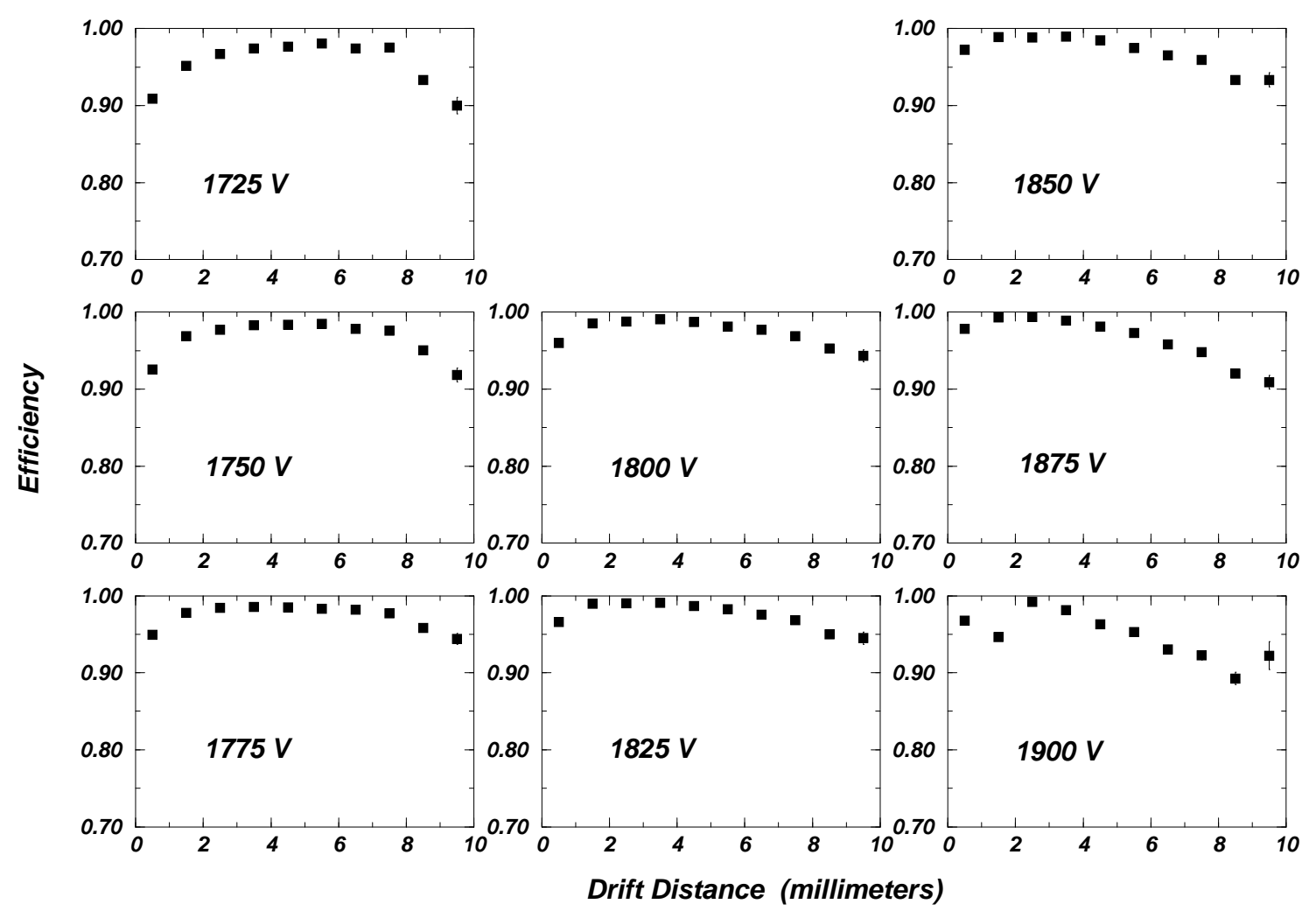

Figure 7.43: Efficiency vs. drift distance using $\mathrm{He}: i-\mathrm{C}_{4} \mathrm{H}_{10}$ [80:20] gas at various high voltages. Notice that the efficiency is generally lower very near to the wire as well as near the cell boundary compared the the middle distances.

chamber are being used for analysis).

For the most part, there was only slight variation between wires. Similar results were found for the other gases tested - variation by more than $20 \%$ was not found.

Now consider a similar efficiency plot to investigate the next possible level of systematic tendencies. In Figure 7.45 efficiency is plotted versus layer number to see if there is a systematic trend in chamber layers that can be seen.

Once again, there is slight variation but no deviation that could be called drastic. Cells in layers $1,4,5$, and 8 are pentagonal while cells in layers $2,3,6$, and 7 are 


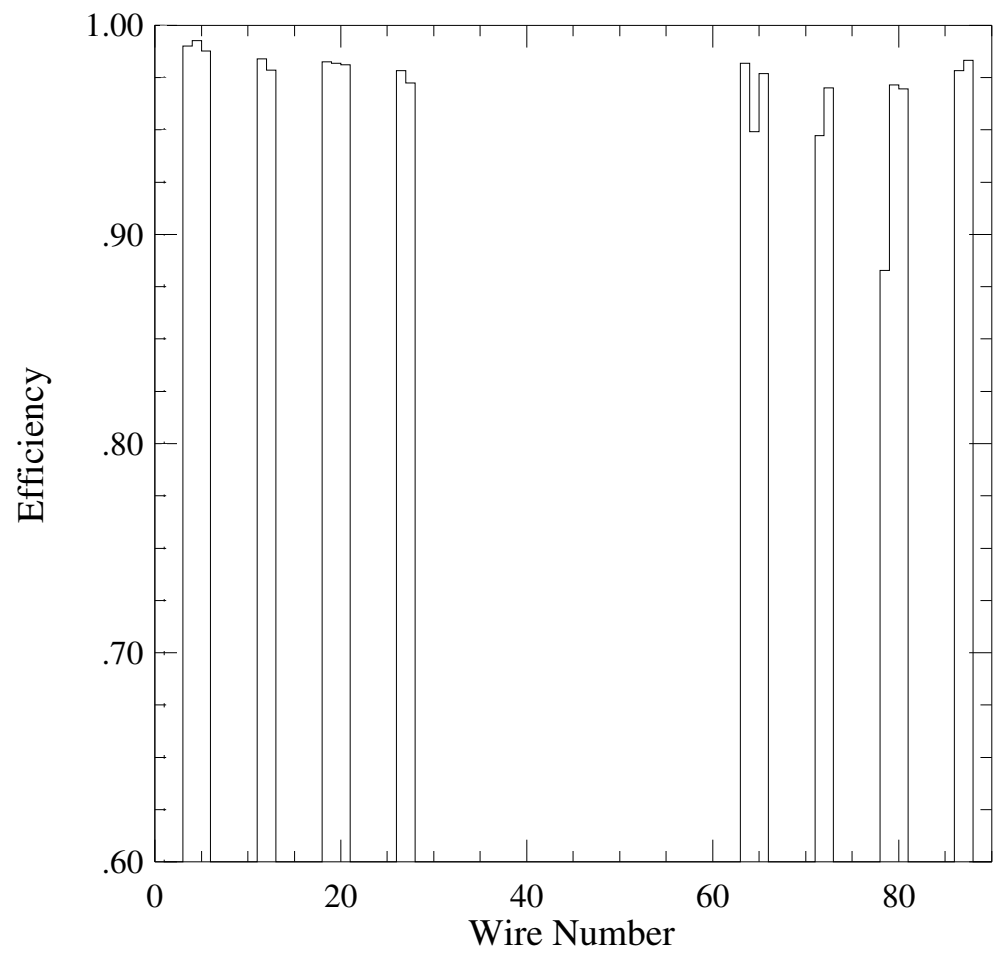

Figure 7.44: Efficiency vs. wire number using $\mathrm{He}: i-\mathrm{C}_{4} \mathrm{H}_{10}[80: 20]$ gas at $1850 \mathrm{~V}$. The blank region in the middle of the plot indicates the stereo layer of wires which were not used in the analysis. The remaining gaps are due to wires that were either not instrumented or were not being considered. From left to right, the left-most groupings represent wires in the first superlayer: layer 1 (pentagonal), layer 2 (hexagonal), layer 3 (hexagonal), and layer 4 (pentagonal). A similar pattern of pentagonal and hexagonal wires applies to the second superlayer of wires which are found on the right-hand side of the large gap. 


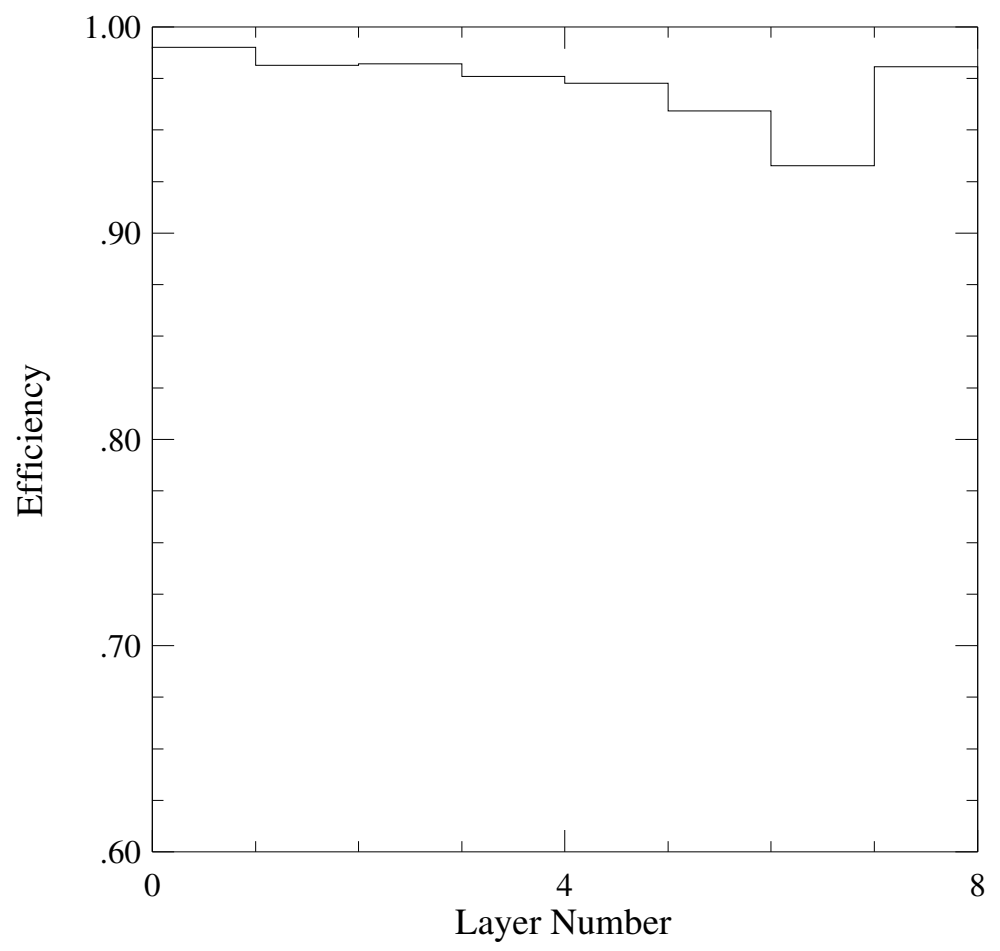

Figure 7.45: Efficiency vs. layer number using $\mathrm{He}: i-\mathrm{C}_{4} \mathrm{H}_{10}[80: 20]$ gas at $1850 \mathrm{~V}$. The numbering scheme for this plot only labels the axial superlayers (the stereo layers are not included). Layers 1 through 4 are for the lower axial superlayer, while 5 through 8 are for the upper axial superlayer.

hexagonal. For the data plotted in Figure 7.45 and on the whole for all gases, the data seems to suggest that hexagonal cells perform marginally poorer than pentagonal cells.

It would also be interesting to see if one of these plots varied in the same manner over all of the runs to see if there is a consistently poor layer or wire. Shown in Figure 7.46 is a plot of efficiency versus layer number for the four gas mixtures averaged over all gases runs. There appears to be some variation in layer efficiency for all gas mixtures, but the efficiency of layer 7 seems to be consistently lower than the rest. No explanation has been found to account for this. In all cases, the efficiency is approximately 0.90 or greater. 


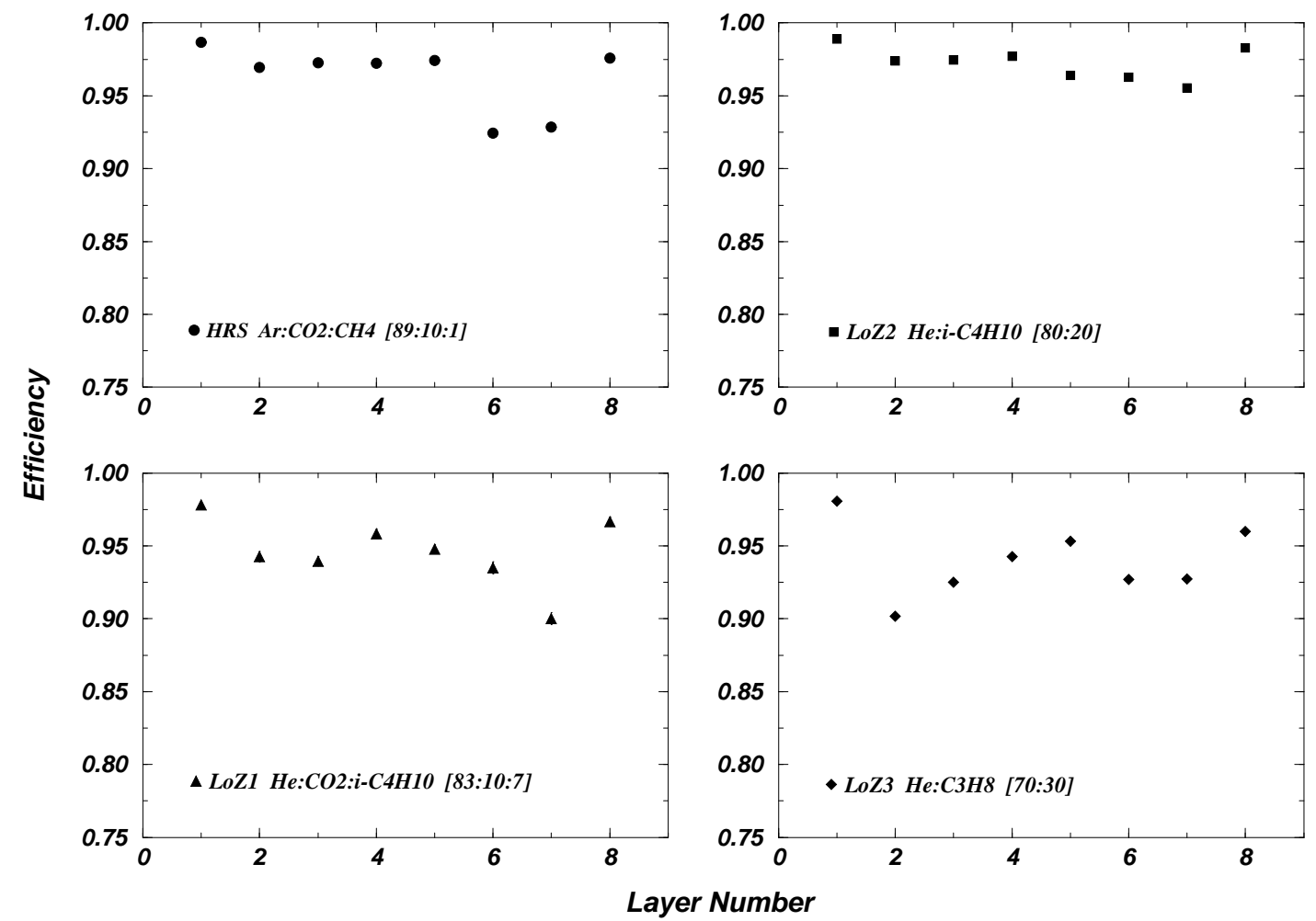

Figure 7.46: Efficiency vs. layer number for the four gas mixtures averaged over all gas runs. The numbering scheme for this plot only labels the axial superlayers (the stereo layers are not included). Layers 1 through 4 are for the lower axial superlayer, while 5 through 8 are for the upper axial superlayer. The error bars are in fact plotted but are small relative to the height of the symbol. 


\section{Chapter 8}

\section{Resolution Studies}

\subsection{Introduction}

After the final tracks have been fit, a second set of studies can be performed which is aimed at determining the spatial track resolution for each of the four chamber gases used: $\mathrm{Ar}: \mathrm{CO}_{2}: \mathrm{CH}_{4}[89: 10: 1], \mathrm{He}: \mathrm{CO}_{2}: i-\mathrm{C}_{4} \mathrm{H}_{10}$ [83:10:7], He: $i-\mathrm{C}_{4} \mathrm{H}_{10}$ [80:20], He: $\mathrm{C}_{3} \mathrm{H}_{8}$ [70:30]. The results from this study will be used in conjunction with the efficiency studies outlined in Chapter 7 to determine in part whether or not helium-based gases are a viable alternative to argon-based gases which have been used extensively in the past. If the helium-based gases do indeed offer an attractive alternative, this study will also help determine which of the helium-based gases should be used. The gas that provides the lowest spatial resolution is desirable because in that case the trajectory of the particle as it traverses the chamber is known as well as possible. Factors that are expected to contribute to spatial resolution include how well the relation between drift time and distance within the chamber is known, or can be approximated, as well as the amount of distortion in the electric field near the cell boundary. Normally, diffusion suffered by the electrons as they drift toward the anode wire contributes significantly to position resolution as mentioned in Chapter 4, but the effects due to electron diffusion are expected to be small [20] since the maximum drift length in the prototype chamber is quite small at $1 \mathrm{~cm}$. In addition, as discussed in Chapter 5, helium-based gases might be expected to provide poorer spatial resolution because of the smaller number 
of primary (and hence, total) ion pairs along the path of the charged particle.

\subsection{Residuals}

Recall from Chapter 6 that drift distance is determined by the track fitting program using TDC information, the $t_{0}$ offset calibration, and the time-distance relation. The quantity that will be used in calculating spatial track resolution is the residual which compares the measured drift distance to that predicted by the track fitting program defined in Chapter 6 as:

residual $=$ measured drift distance - predicted drift distance.

Shown in Figure 8.47 is the distribution of the raw residual values for the entire cell. The peak around $0 \mu \mathrm{m}$ indicates that the drift distances obtained from the TDC and the track fitting algorithm average to $0 \mu \mathrm{m}$ with no significant offset or bias.

To better understand the regions where the two measures of the drift distance do not agree well, consider taking distance slices through the cell, placing the raw residuals in $1 \mathrm{~mm}$ wide bins progressively further from the sense wire as shown in Figure 8.48.

In each case, these residual values will then be fit to a Gaussian distribution and further analyzed in order to learn how accurately the track fitting program is able to place the trajectory of the particle within the chamber.

\subsection{Fitting Residuals}

The average cell resolution is found by fitting the residuals shown in Figure 8.47 to a single Gaussian distribution. The likelihood fit algorithm within the MINUIT [27] fitting package was used as opposed to the $\chi^{2}$ algorithm in order to treat the errors in bins with small counts properly. This plot is shown in Figure 8.49. Resolution is 


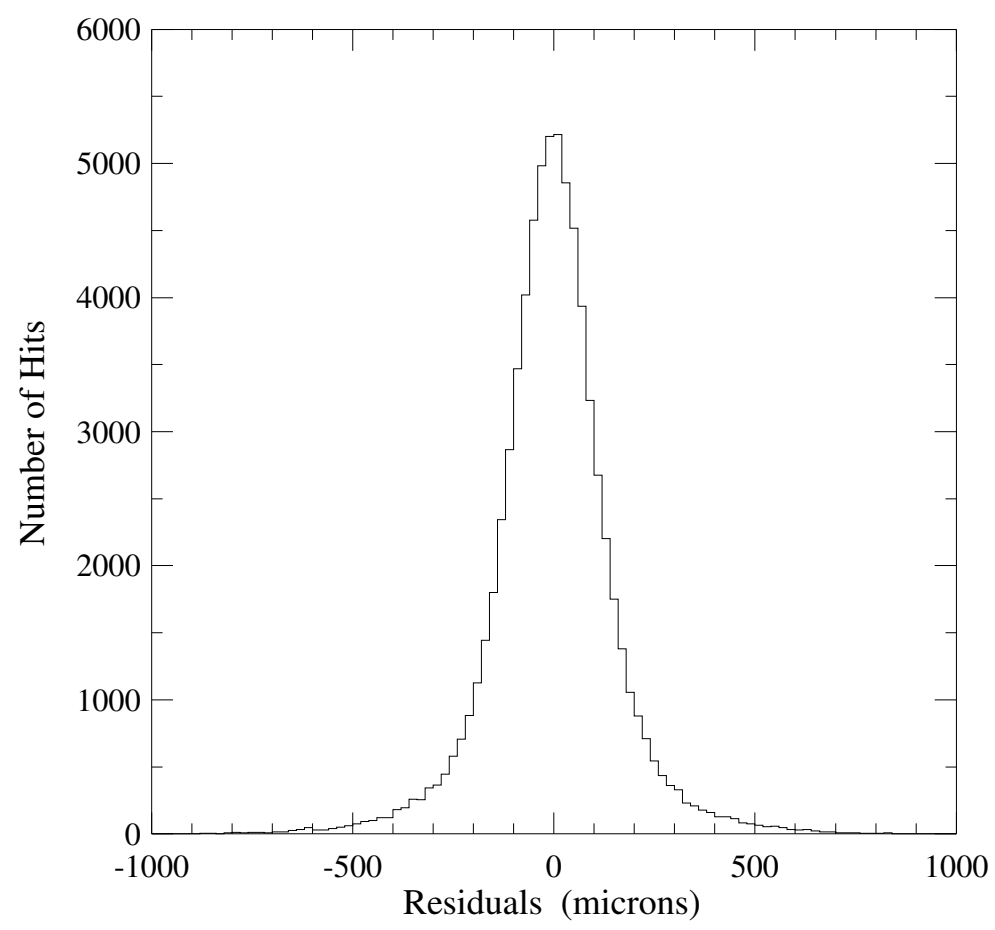

Figure 8.47: Histogram of residuals for each hit in the $\mathrm{He}: i-\mathrm{C}_{4} \mathrm{H}_{10}$ [80:20] gas data sample at $1850 \mathrm{~V}$ data sample before fitting to a Gaussian distribution.

defined as the standard deviation of this distribution, and the error in the resolution is simply the statistical error in the standard deviation.

Obviously, the distribution shown in Figure 8.49 is not governed by a purely Gaussian shape. Assuming that the peak region follows a Gaussian distribution, the regions with large residual values can be excluded so that the fitting algorithm can concentrate on fitting the peak which is of main concern. However, the question arises: Where should the cut be made? After considering many plots similar to those shown in Figure 8.50 it was decided that the residual values should be fit to a Gaussian distribution where the regions further than $1.5 \sigma$ on either side of the mean are excluded in order to ignore non-Gaussian tail contributions. The decision to cut so close to the mean 


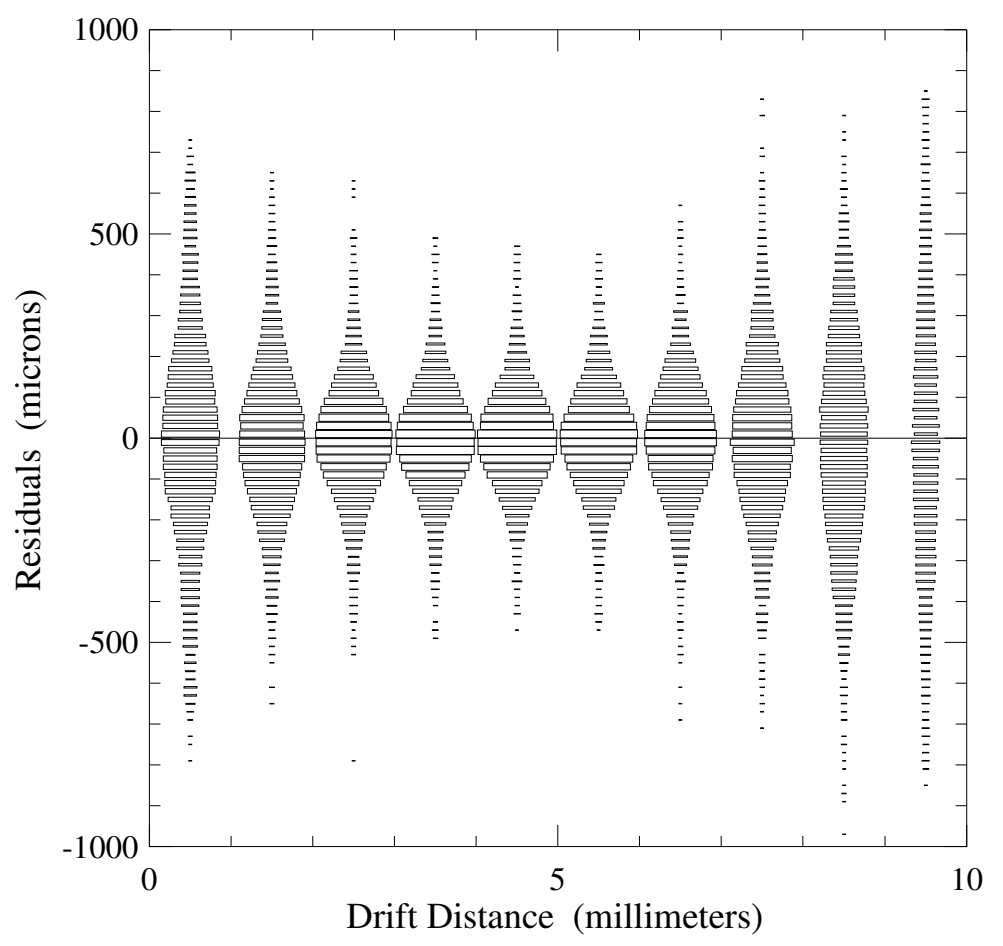

Figure 8.48: Residuals vs. the absolute value of the predicted drift distance obtained through the fitting program using $\mathrm{He}: i-\mathrm{C}_{4} \mathrm{H}_{10}[80: 20]$ gas at $1850 \mathrm{~V}$. The number of counts with a given residual and drift distance is indicated by the thickness and width of the bars. For most regions of interest in the cell, the residuals are distributed about $0 \mu \mathrm{m}$ in a roughly Gaussian distribution. At large distances from the sense wire, the residuals are substantially larger than those closer to the wire.

was arrived at after considering how well the peaks were fit; or, alternatively, the decision was arrived at after considering how poorly the peaks were fit when the data was truncated at $2 \sigma$ from the mean.

Clearly, the fit using the cutoff at $1.5 \sigma$ is the better fit of the two- the $\chi^{2} /$ degree of freedom ${ }^{1}(d o f)$ in the case where we use the $1.5 \sigma$ cut is 1.99 while the $\chi^{2} /$ dof in the case of the cut at $2 \sigma$ is 8.61 . Obviously, the more data points one leaves out in the

\footnotetext{
${ }^{1}$ The term degrees of freedom is defined to be the number of data points minus the number of coefficients plus one constant term used to constrain the fit [24]. A fit to a single Gaussian distribution has 3 associated fit parameters, whereas a fit to two Gaussian distributions has 6 .
} 


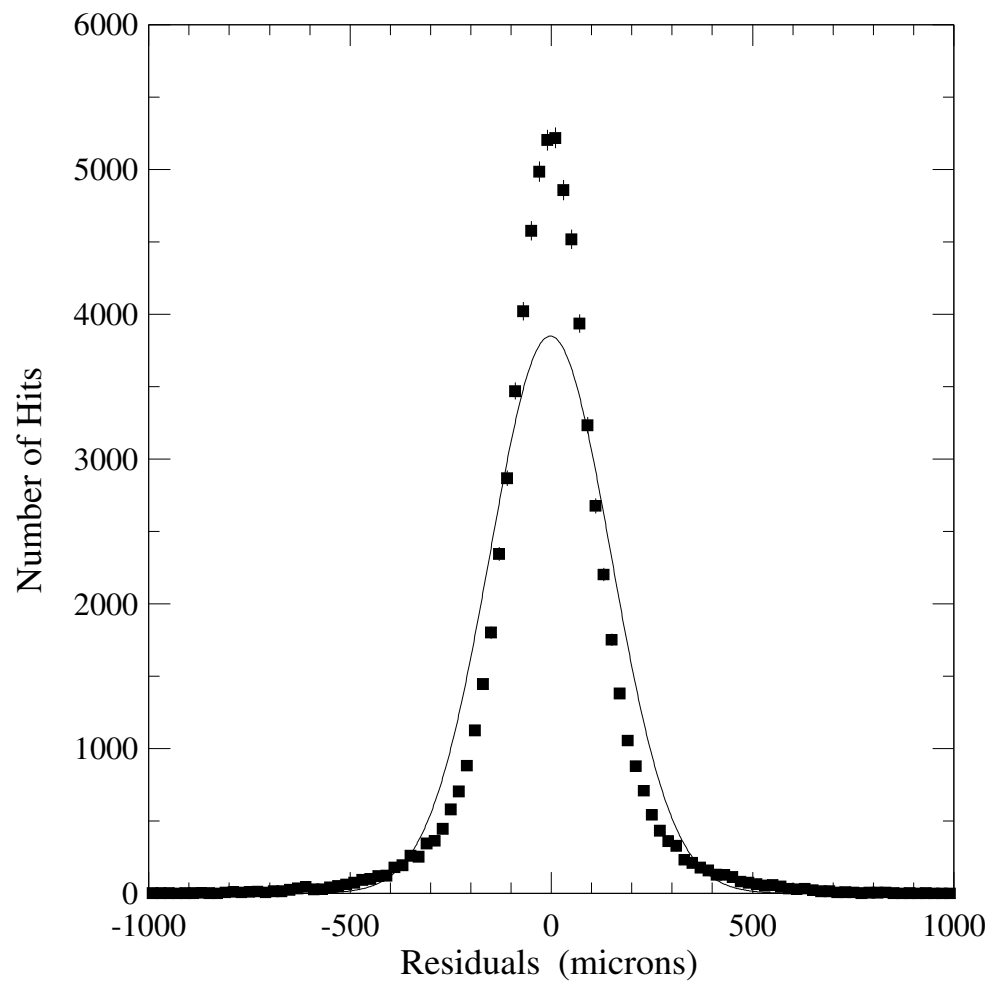

Figure 8.49: Histogram of the residuals for each hit using $\mathrm{He}: i-\mathrm{C}_{4} \mathrm{H}_{10}$ [80:20] gas at $1850 \mathrm{~V}$. It is quite clear that on the whole the residual values are not well described by a single Gaussian distribution, and that the regions with large residual values, the "tails" of the distribution, are adversely affecting the fit of the peak.

tails of the distribution, the better the amplitude of the remaining data in the peak will be fit, but it was felt that $1.5 \sigma$ was a suitable trade off between cutting out data and obtaining a good estimate of the resolution as the width of the residual peak. The justification for excluding the non-Gaussian tail regions of the residual data is that hits in these regions are not well measured. Systematic effects due at cell boundaries may be the cause, but in any case, data in these outlying regions do not constitute a significant fraction of the complete data sample, and they are not interesting for these studies. 
(a)
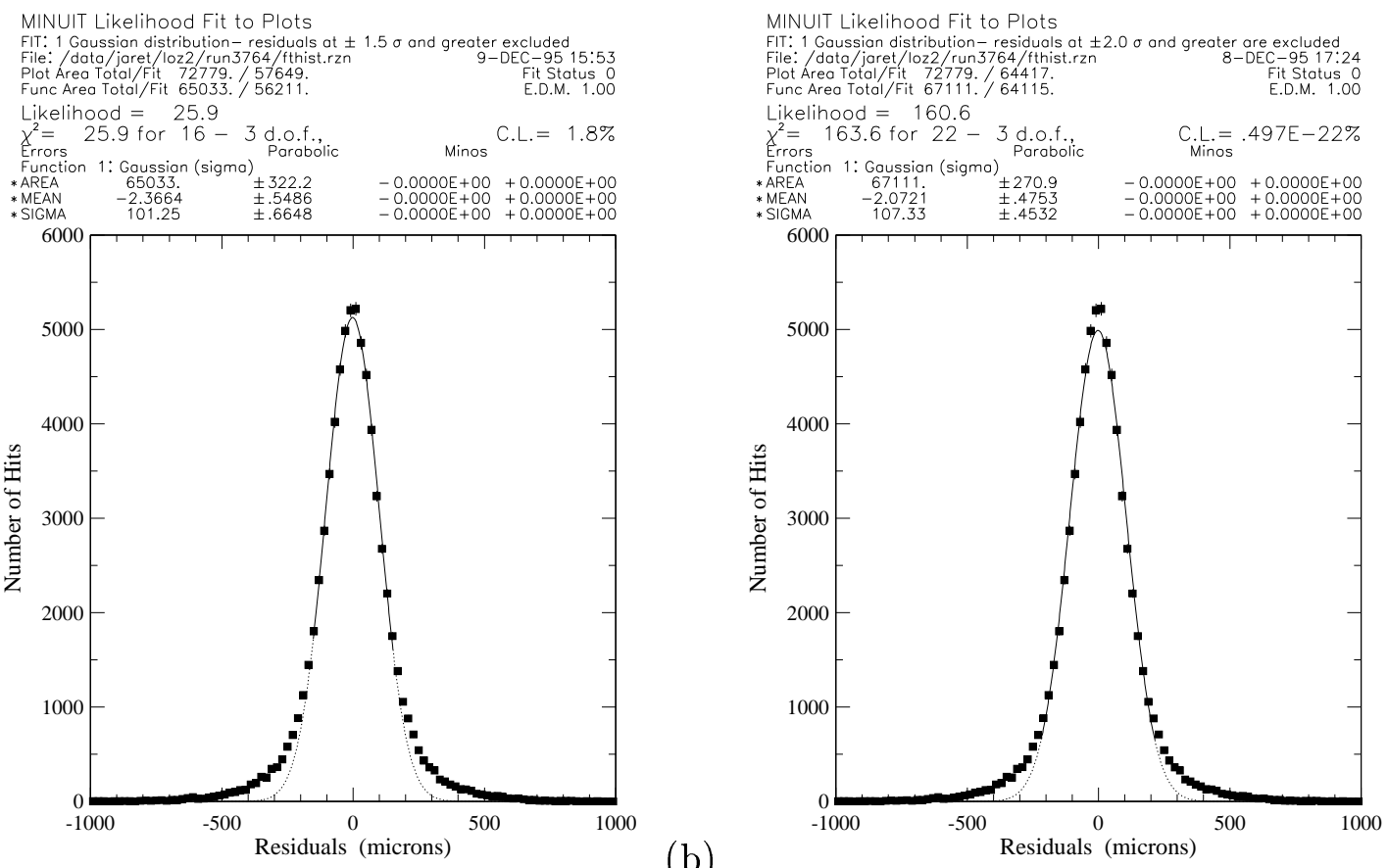

Figure 8.50: Comparison of fit residual distributions when regions further than (a) $1.5 \sigma$ and (b) $2.0 \sigma$ are excluded using $\mathrm{He}: i-\mathrm{C}_{4} \mathrm{H}_{10}[80: 20]$ gas at $1850 \mathrm{~V}$. Plot (a) on the right shows the residual values fitted to a single Gaussian distribution excluding regions further than $1.5 \sigma$ on either side of the mean, while plot (b) on the left shows the same residual values fitted to the same single Gaussian distribution except that in this case regions beyond $2.0 \sigma$ are excluded. Plot (a) is the better fit because it describes the amplitude (height) of the peak much better. The dotted part of the best fit curve shows the region that has been excluded by the cuts.

Also investigated was the possibility of fitting the residual data to two Gaussian distributions - one narrow Gaussian distribution to describe the dominant peak while the second, broader distribution that would account for the non-Gaussian tails.

Comparing the fit to a single Gaussian distribution with regions at $1.5 \sigma$ and further have been excluded to the fit to 2 Gaussian distributions where no regions have been excluded, it is evident that the fits are comparable. The $\chi^{2} /$ dof in the case of the single Gaussian distribution is 1.99 while for the fit to 2 Gaussian distributions, the $\chi^{2} /$ dof is 
(a)
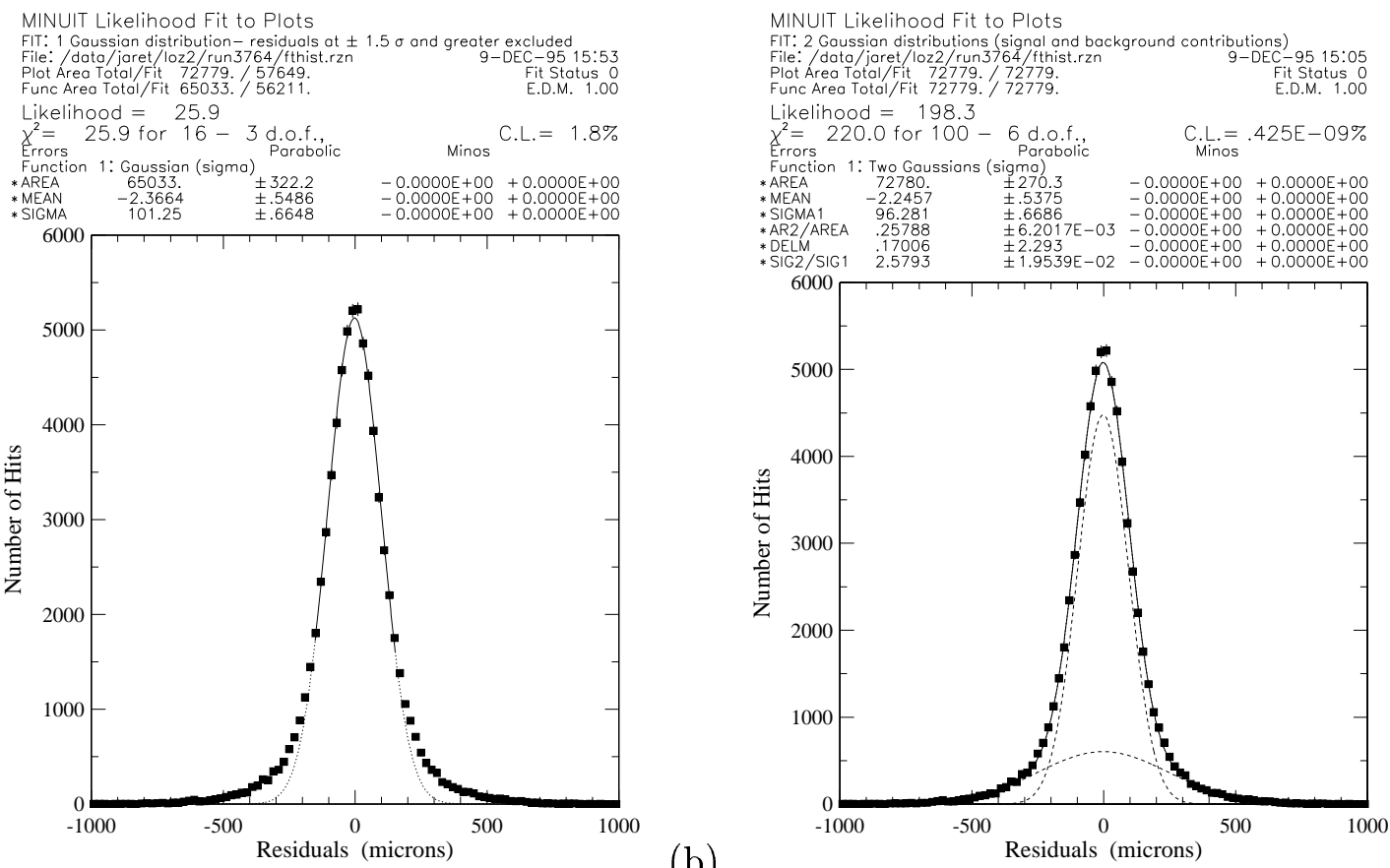

Figure 8.51: Comparison of the same residual values fit to (a) 1 or (b) 2 Gaussian distributions using $\mathrm{He}: i-\mathrm{C}_{4} \mathrm{H}_{10}[80: 20]$ gas at $1850 \mathrm{~V}$. Notice that the both scenarios, plots (a) and (b), fit the data well. The dashed curves in plot (b) showing the fit to 2 Gaussian distributions illustrates the relative contribution of the background to the overall shape.

2.34. However, as a fitting algorithm, the 2 Gaussian method needed more maintenance than the single Gaussian fit. Placing only the constraint that the area of each of the curves be greater than zero, the two-Gaussian method had difficulty correctly identifying both the background distribution and the peak well, especially in the case of $\mathrm{He}: \mathrm{CO}_{2}: i-\mathrm{C}_{4} \mathrm{H}_{10}[83: 10: 7]$ where the data set was smaller than the rest and there was greater fluctuation in the curve. To correct this, additional constraints were provided such as the ratio of the areas of the two curves and the ratios of the standard deviations of the two curves. As a consequence, though, our resolution becomes somewhat model dependent using the method of fitting to 2 Gaussian distributions. Therefore, the 
more reliable method of using a single Gaussian distribution to describe the data was employed throughout the subsequent resolution analysis.

\subsection{Resolution Results}

Based on the above arguments, the average cell resolution was determined by fitting the residual data to a single Gaussian distribution where regions further than $1.5 \sigma$ on either side of the mean were excluded, in conjunction with the analysis cuts outlined in Chapter 7, section 7.4. The plots provided in Figure 8.52 show typical residual values being fit by the method established above. The average cell resolution is obtained by considering the residuals from all the hits in a given run. In subsequent analysis, the resolution in different regions of the cell can be determined by placing certain cuts on the data that isolate the areas in question.

One of the first questions that needs to be answered for each gas is where the optimum high voltage operating region is. This is done by examining the spatial resolution obtained at each voltage. Different gas mixtures will contribute to different spatial resolutions at different high voltages; the results of this study for all four gases used in the chamber are summarized in Table 8.5 and plotted in Figure 8.53. The graph clearly shows the trend of improving resolution as high voltage increases for

each gas mixture. In the case of $\mathrm{Ar}: \mathrm{CO}_{2}: \mathrm{CH}_{4}[89: 10: 1]$ and $\mathrm{He}: i-\mathrm{C}_{4} \mathrm{H}_{10}$ [80:20] gases, as the voltage increases resolution continues to improve up to a minimum value, after which it begins to increase. For the $\mathrm{He}: \mathrm{CO}_{2}: i-\mathrm{C}_{4} \mathrm{H}_{10}[83: 10: 7]$ and $\mathrm{He}: \mathrm{C}_{3} \mathrm{H}_{8}[70: 30]$ gas mixtures the higher voltages were not investigated in order to find this absolute minimum because it was already evident from the efficiency studies that higher voltages had markedly decreased efficiencies. Overall, $\mathrm{He}: i-\mathrm{C}_{4} \mathrm{H}_{10}[80: 20]$ gas appears to have the best average cell resolution, reaching a resolution of $(96.8 \pm 0.7) \mu \mathrm{m}$ at $1875 \mathrm{~V}$, 
(a)
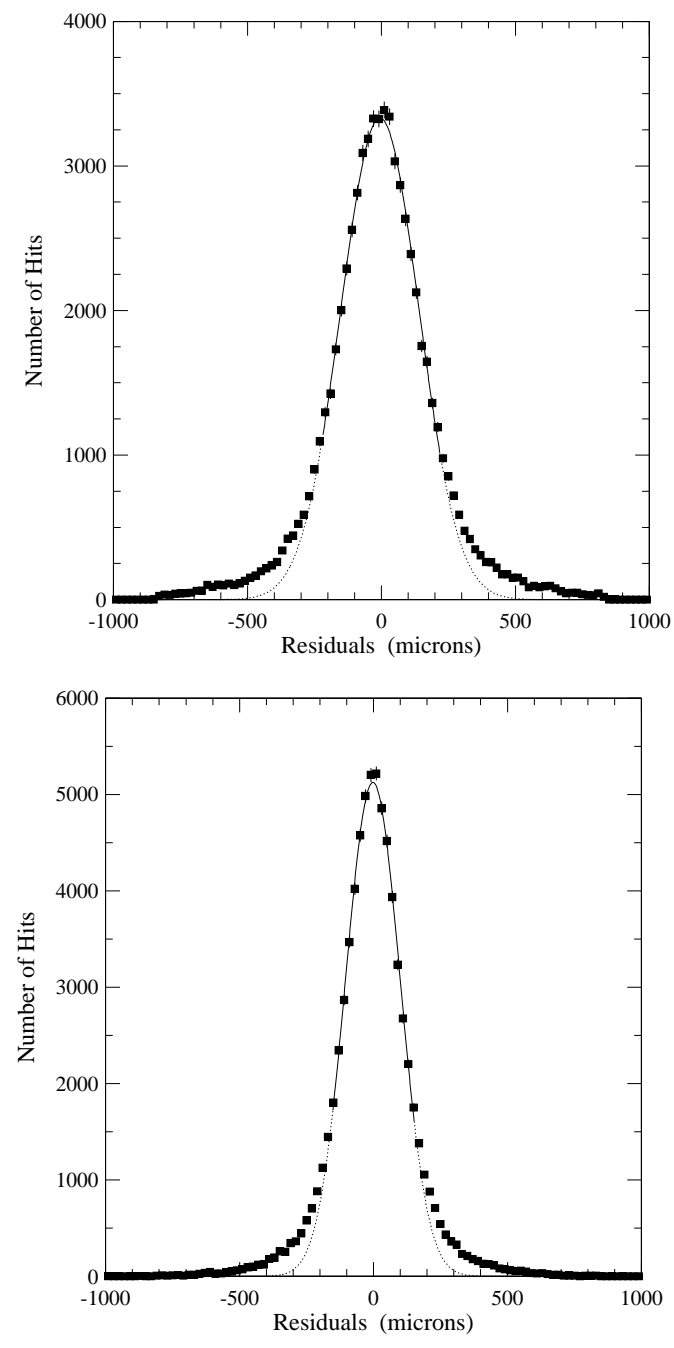

(b)
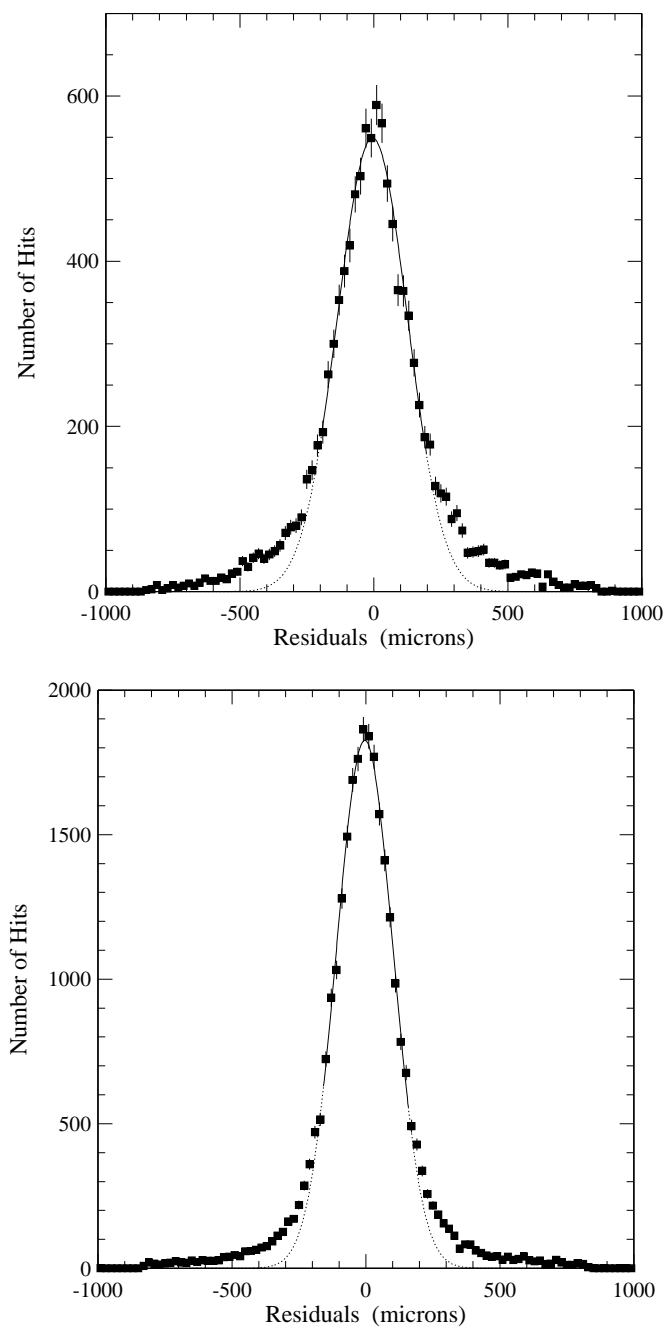

(d)

Figure 8.52: Comparison of typical residual values for the four gases tested in the prototype fit using a single Gaussian distribution where regions further than $1.5 \sigma$ on either side of the mean were excluded. Plot (a) shows the results for Ar: $\mathrm{CO}_{2}: \mathrm{CH}_{4}$ [89:10:1] gas in the upper left-hand corner, plot (b) shows the results for $\mathrm{He}: \mathrm{CO}_{2}: i-\mathrm{C}_{4} \mathrm{H}_{10}$ [83:10:7] gas in the upper right-hand corner, plot (c) shows $\mathrm{He}: i-\mathrm{C}_{4} \mathrm{H}_{10}$ [80:20] gas in the lower left-hand corner, while plot (d) shows $\mathrm{He}: \mathrm{C}_{3} \mathrm{H}_{8}$ [70:30] gas in the lower right-hand corner. Recall that the standard deviation (width) of each of these distributions is defined as the resolution for that particular gas run. 
while $\mathrm{He}: \mathrm{C}_{3} \mathrm{H}_{8}[70: 30]$ gas is comparable with a resolution of $(100.3 \pm 1.2) \mu \mathrm{m}$ at 1950 V. Notice that $\mathrm{He}: \mathrm{C}_{3} \mathrm{H}_{8}[70: 30]$ gas requires a substantially higher running voltage than the other gases tested.

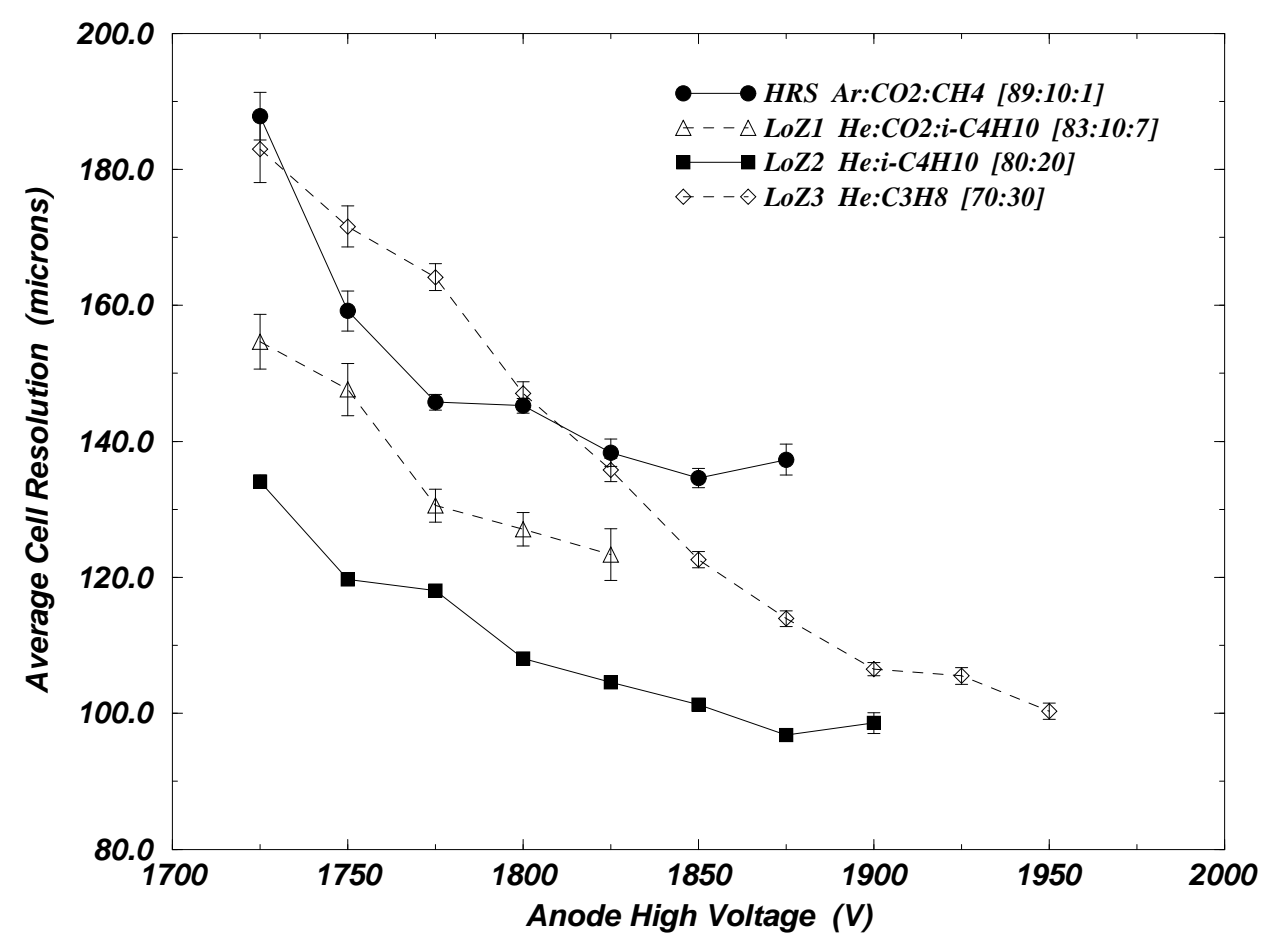

Figure 8.53: Average resolution vs. anode high voltage for all gases used in the prototype chamber. Note that over most of the high voltage range, helium-based gases have a better resolution than the argon-based gas. In particular, $\mathrm{He}: i-\mathrm{C}_{4} \mathrm{H}_{10}$ [80:20] gas has the best average cell resolution of all, closely followed by $\mathrm{He}: \mathrm{C}_{3} \mathrm{H}_{8}$ [70:30] gas when considered in the appropriate optimal high voltage region.

A second investigation studies how resolution depends on track location within the cell. Starting with the entire residual data, a cut was placed on predicted drift distance to isolate residuals for each region of the cell in bins $1 \mathrm{~mm}$ wide. These residual values were then fit to a single Gaussian distribution excluding the region beyond $1.5 \sigma$ on 


\begin{tabular}{|c|c|c|}
\hline Gas Mixture & $\begin{array}{c}\text { Anode } \\
\text { High Voltage } \\
(\mathrm{V})\end{array}$ & $\begin{array}{c}\text { Average } \\
\text { Cell Resolution } \\
\text { (microns) }\end{array}$ \\
\hline Ar: $\mathrm{CO}_{2}: \mathrm{CH}_{4}[89: 10: 1]$ & $\begin{array}{l}1725 \\
1750 \\
1775 \\
1800 \\
1825 \\
1850 \\
1875 \\
\end{array}$ & $\begin{array}{l}187.8 \pm 3.5 \\
159.2 \pm 2.9 \\
145.7 \pm 1.2 \\
145.2 \pm 1.1 \\
138.3 \pm 2.0 \\
134.6 \pm 1.4 \\
137.3 \pm 2.3\end{array}$ \\
\hline $\mathrm{He}: \mathrm{CO}_{2}: i-\mathrm{C}_{4} \mathrm{H}_{10}[83: 10: 7]$ & $\begin{array}{l}1725 \\
1750 \\
1775 \\
1800 \\
1825\end{array}$ & $\begin{array}{l}154.7 \pm 4.0 \\
147.6 \pm 3.9 \\
130.6 \pm 2.4 \\
127.1 \pm 2.5 \\
123.3 \pm 3.8\end{array}$ \\
\hline $\mathrm{He}: i-\mathrm{C}_{4} \mathrm{H}_{10}[80: 20]$ & $\begin{array}{l}1725 \\
1750 \\
1775 \\
1800 \\
1825 \\
1850 \\
1875 \\
1900\end{array}$ & $\begin{array}{c}134.1 \pm 1.0 \\
119.7 \pm 0.8 \\
118.0 \pm 0.7 \\
108.0 \pm 0.7 \\
104.6 \pm 0.7 \\
101.3 \pm 0.6 \\
96.8 \pm 0.7 \\
98.56 \pm 1.6\end{array}$ \\
\hline $\mathrm{He}: \mathrm{C}_{3} \mathrm{H}_{8}[70: 30]$ & $\begin{array}{l}1725 \\
1750 \\
1775 \\
1800 \\
1825 \\
1850 \\
1875 \\
1900 \\
1925 \\
1950\end{array}$ & $\begin{array}{l}183.0 \pm 4.9 \\
171.6 \pm 3.0 \\
164.1 \pm 2.0 \\
147.0 \pm 1.7 \\
135.8 \pm 1.7 \\
122.6 \pm 1.2 \\
113.9 \pm 1.1 \\
106.5 \pm 1.0 \\
105.5 \pm 1.2 \\
100.3 \pm 1.2\end{array}$ \\
\hline
\end{tabular}

Table 8.5: Table of results indicating the average cell resolution for the four chamber gases at various operating voltages. 
either side of the mean, and the resolution was taken to be the standard deviation as before. Resolution near the sense wire as well as far from the sense wire (near the cell boundary) was expected to be poorer than the average resolution over the whole cell. In the case of the former, electron avalanches have not had a chance to build up which in turn means that knowledge of time breaks down very close to the sense wire and becomes dominated by noise. While, in the latter case, the resolution will be poor partly due to the longer drift length the electrons had to drift as they move toward the anode wires mentioned in Chapter 4, and also because of the distortions in the electric field at the cell boundaries, as discussed by S. Uno et al. [20]. Diffusion in a large cell contributes to a decrease in resolution because the more diffuse the electrons are when they reach the anode, the larger the change in path the outlying electrons have to travel. As a consequence, there will not be a sharp leading edge for the anode pulse. The TDC will have to wait longer until sufficient charge has built up, which is a longer time than if the electrons were not diffuse. Hence, there is a larger difference between the fitted drift distance and the drift distance determined from the TDC. In small cells, the electric field in the corners of the cells tends to be irregular, which in turn leads to poor charge collection at the anode. Figure 8.54 shows the dependence of resolution on distance from the sense wire where resolution has been averaged over $1 \mathrm{~mm}$ wide distance bins and assigned the associated mean drift distance.

As expected, the resolution near the sense wire (drift distance $=0-1 \mathrm{~mm}$ ) and near the cell boundary (drift distance $=8-9 \mathrm{~mm}$ ) is substantially higher than in the middle part of the cell where resolution values as good as $(76.7 \pm 3.0) \mu \mathrm{m}$ are seen (using He: $i-\mathrm{C}_{4} \mathrm{H}_{10}[80: 20]$ gas at $\left.1900 \mathrm{~V}\right)$.

The dependence of resolution upon layer was also investigated; that is, were certain layers showing intrinsically poorer resolution than others? The plots shown in Figure 8.55 seem to indicate that this was not the case. The shapes of the the resolution 


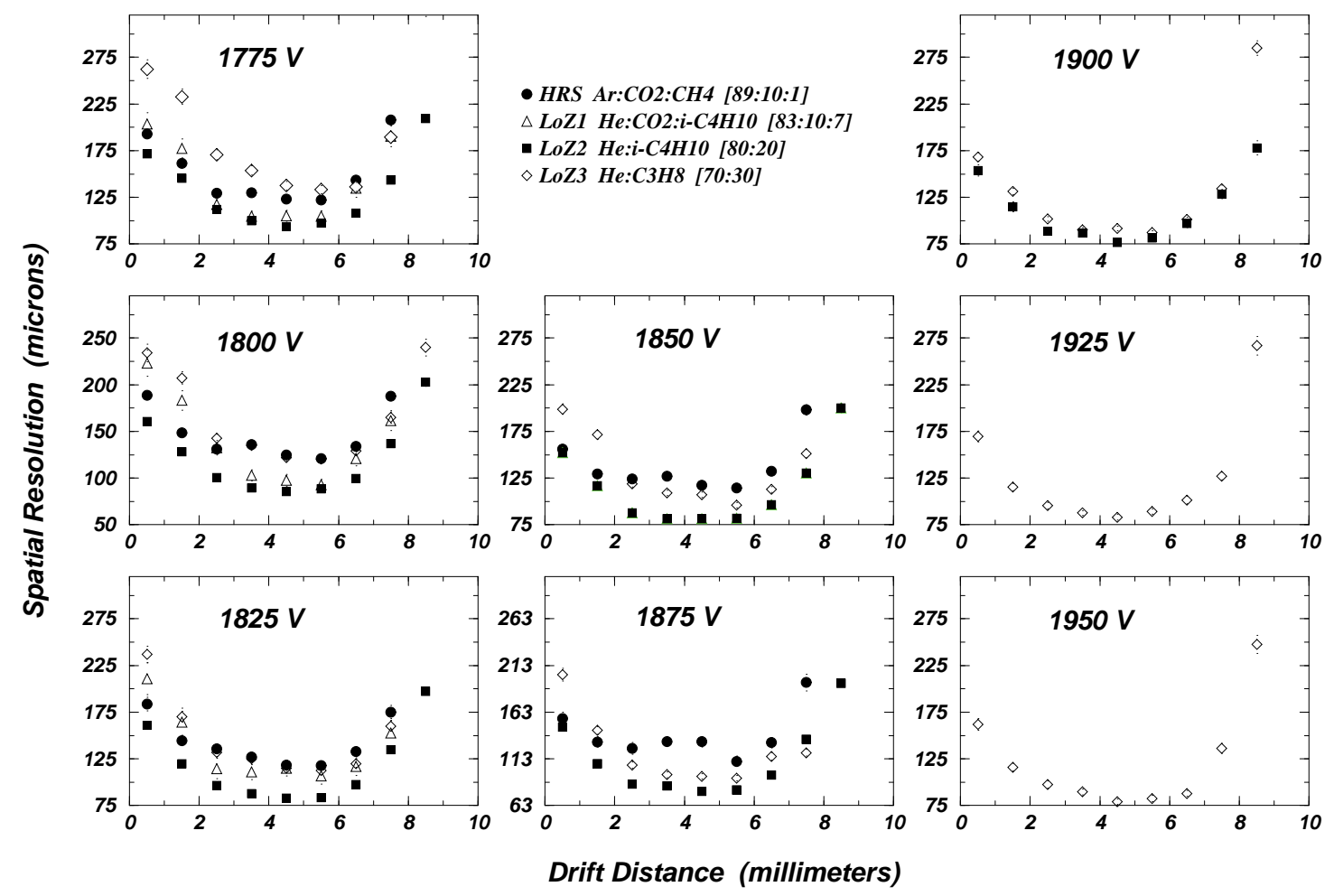

Figure 8.54: Resolution vs. drift distance for all gases tested in the prototype at various anode high voltages. The resolution is poorer at small and large radial distances from the sense wire, but can reach values as good as $(76.7 \pm 3.0) \mu \mathrm{m}$ in the middle region of the cell (using $\mathrm{He}: i-\mathrm{C}_{4} \mathrm{H}_{10}$ [80:20] gas at $1900 \mathrm{~V}$ ). The error bars have been left off this plot in order to make it easier to read. Typical error bars are less than $4 \%$.

versus drift distance curves are all similar regardless of layer, which means that there is very little dependence of resolution upon layer in the prototype drift chamber.

Finally, a less biased method of determining spatial resolution was considered in the case of the $\mathrm{He}: i-\mathrm{C}_{4} \mathrm{H}_{10}[80: 20]$ gas mixture. Using the "refit" tracks that were discussed in Section 6.6 where residual values are found by dropping each hit in the track, a new calculation of the resolution was performed. Also recall from Section 6.6 that the estimate in the track error is determined on a layer-by- layer basis. In order to obtain the average resolution as a function of drift distance for a particular gas run, 

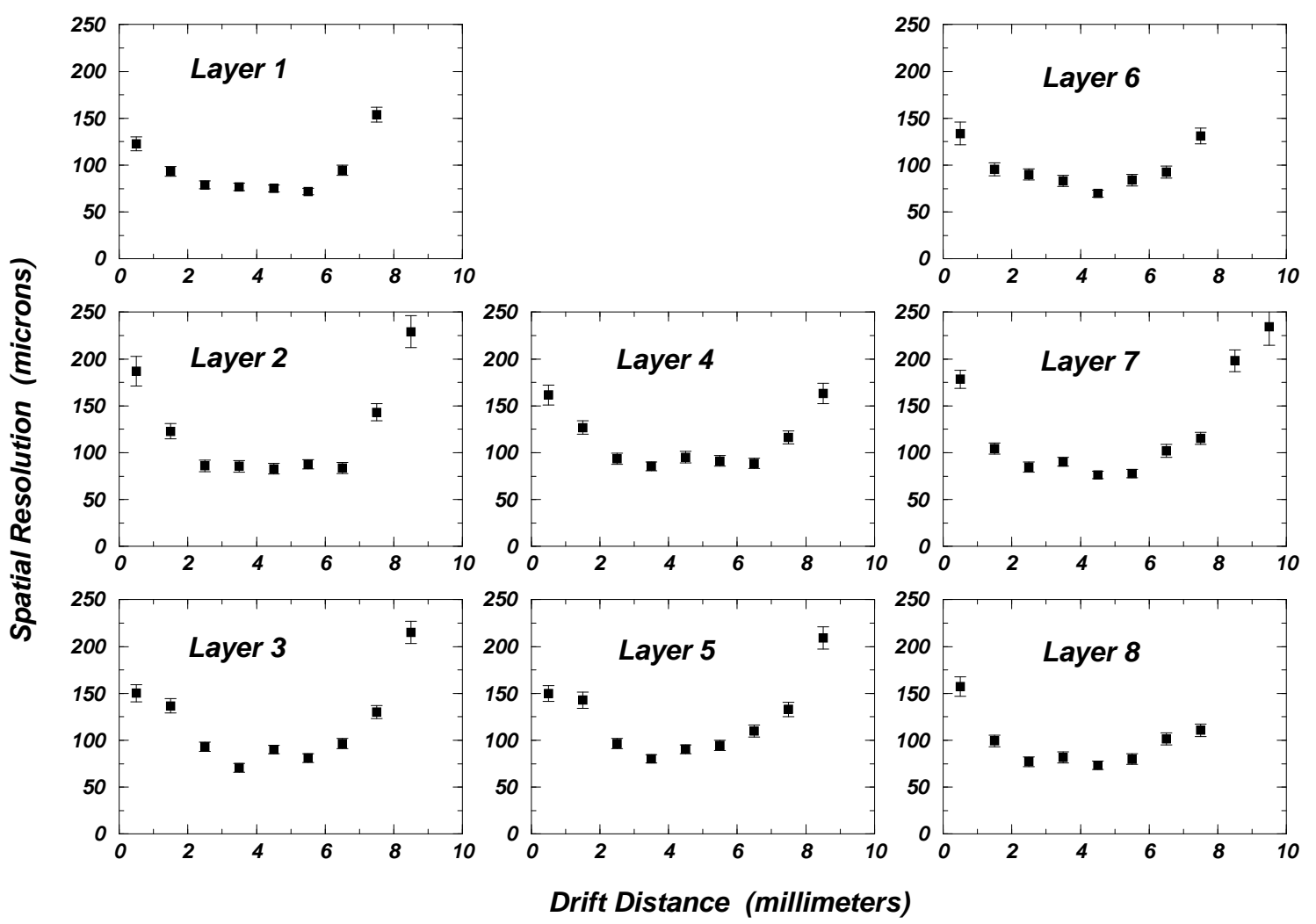

Figure 8.55: Resolution vs. drift distance for each axial layer in the prototype chamber using He: $i-\mathrm{C}_{4} \mathrm{H}_{10}[80: 20]$ gas at $1850 \mathrm{~V}$. Numbering from the bottom of the chamber, layer 1 is the layer closest to the IP while layer 8 is the farthest; layers 1-4 make up the first superlayer, while layers 5-8 comprise the second superlayer. Stereo layers are not included in the numbering scheme, because they are not analyzed. There is very little difference between the resolution spectra for the different layers. Similar plots are obtained for the other gases tested. 
the mean [24] of the resolutions was calculated at a given drift distance over all 8 layers used in the analysis:

$$
\mu_{\text {resolution }}=\frac{\sum_{i=1}^{8} \frac{x_{i}}{\sigma_{i}^{2}}}{\sum_{i=1}^{8} \frac{1}{\sigma_{i}^{2}}}
$$

where the associated error in the above mean resolution value is:

$$
\sigma_{\mu}=\sqrt{\sum_{i=1}^{8} \frac{1}{\sigma_{i}^{2}}}
$$

Figure 8.4 shows a plot of the less biased spatial resolution versus drift distance comparing results from the original resolution analysis in which hits were not dropped with the results from this new method. Notice that in the middle region of the cell, the new resolution values are, in general, slightly higher than the original calculations, while near the anode wire and near the cell boundary, the less biased method yields lower resolution values. This trend is expected due to more weight being placed on hits that have corresponding drift distances in the middle part of the cell. A hit near the anode wire or near the cell boundary is weighted less, and as a consequence, the associated residual is not affected very much when the hit is dropped. The result is that the new resolution values near the anode and near the cell boundary are slightly less than those from the original analysis due to the subtraction of the estimate of the track error. Whereas, a hit in the middle of the cell tends to pull the fit toward itself more strongly; therefore, when it is dropped, the residual value typically becomes slightly larger and contributes to a larger value for the resolution. 


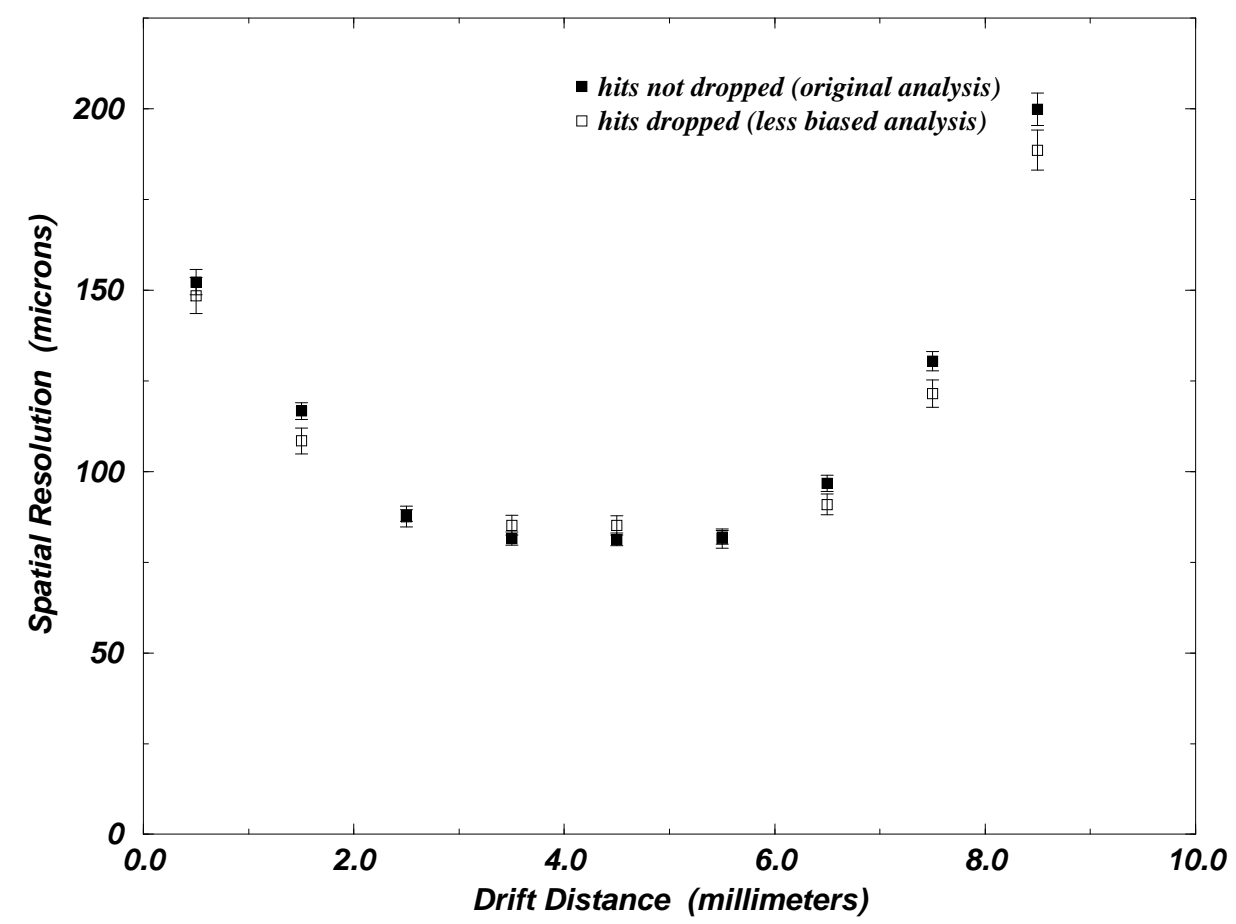

Figure 8.56: Resolution vs. drift distance averaged over all layers in the prototype chamber using He: $i-\mathrm{C}_{4} \mathrm{H}_{10}$ [80:20] gas at $1850 \mathrm{~V}$ making use of the less biased residuals obtained from dropping hits in turn and refitting the tracks. Generally, the unbiased method gives slightly higher resolution values in the middle part of the cell, while providing slightly lower resolution values near the anode wire and cell boundary. 


\section{Chapter 9}

\section{Conclusion}

In order to minimize multiple scattering, the design of the drift chamber for the $B_{A} B_{A R}$ detector has been guided throughout its development by the need to reduce the amount of material used. The issue of a low mass chamber has lead the drift chamber group to consider helium-based gases as an alternative to the more traditional argon-based gas mixtures used previously in many drift chambers. A full-length prototype drift chamber was constructed at SLAC so that the viability of helium as a drift chamber gas might be tested. The focus of this thesis has been to examine the performance of various helium gas mixtures and compare those results to the values obtained for argon-based gas mixtures.

\subsection{Optimum High Voltage}

In order to compare all four gas mixtures properly, the high voltage region where each gas performs best must first be identified. Once that is done, the relative merits of each gas at a certain efficiency and resolution can then be compared and the choice for best gas can be made.

The optimum high voltage was chosen based on two criteria: chamber efficiency and cell spatial resolution. There was no hard and fast way to do this except make a judgment based on weighing the two factors. The optimum high voltage regions chosen are quoted in Table 9.6 below. 


\begin{tabular}{|l|c|c|c|}
\hline Gas Mixture & $\begin{array}{c}\text { Anode } \\
\text { High Voltage } \\
(\mathbf{V})\end{array}$ & $\begin{array}{c}\text { Average } \\
\text { Efficiency }\end{array}$ & $\begin{array}{c}\text { Average Cell } \\
\text { Resolution } \\
\text { (microns) }\end{array}$ \\
\hline \hline $\mathrm{Ar}: \mathrm{CO}_{2}: \mathrm{CH}_{4}[89: 10: 1]$ & 1800 & $0.9699 \pm 0.0006$ & $145.2 \pm 1.1$ \\
$\mathrm{He}: \mathrm{CO}_{2}: i-\mathrm{C}_{4} \mathrm{H}_{10}[83: 10: 7]$ & 1775 & $0.9587 \pm 0.0019$ & $130.5 \pm 2.4$ \\
$\mathrm{He}: i-\mathrm{C}_{4} \mathrm{H}_{10}[80: 20]$ & 1850 & $0.9741 \pm 0.0006$ & $101.2 \pm 0.7$ \\
$\mathrm{He}: \mathrm{C}_{3} \mathrm{H}_{8}[70: 30]$ & 1925 & $0.9818 \pm 0.0008$ & $105.5 \pm 1.2$ \\
\hline
\end{tabular}

Table 9.6: Table of results indicating the optimum operating high voltage for each of the four gas mixtures tested in the prototype drift chamber.

Table 9.6 shows that $\mathrm{He}: \mathrm{C}_{3} \mathrm{H}_{8}[70: 30]$ gas has the best efficiency, closely followed by the $\mathrm{He}: i-\mathrm{C}_{4} \mathrm{H}_{10}[80: 20]$ gas mixture; whereas $\mathrm{He}: i-\mathrm{C}_{4} \mathrm{H}_{10}[80: 20]$ gas has the best spatial resolution, with $\mathrm{He}: \mathrm{C}_{3} \mathrm{H}_{8}$ [70:30] being very comparable. With respect to efficiency, the argon-based gas performed reasonably well, but was the poorest of all gases with regard to spatial resolution. The percentage difference in efficiency between $\mathrm{He}: \mathrm{C}_{3} \mathrm{H}_{8}$ [70:30] gas and $\mathrm{He}: i-\mathrm{C}_{4} \mathrm{H}_{10}[80: 20]$ is only $0.8 \%$, but, on the other hand, the percentage difference in resolution between those two gas mixtures is $4.3 \%$. However, since the deviations in efficiency are expected to be smaller than those for resolution, these values are not a good indication of the best gas mixture.

Consider now the plot of efficiency versus radius at the optimum high voltage for each gas. It was evident from Table 9.6 that $\mathrm{He}: \mathrm{C}_{3} \mathrm{H}_{8}[70: 30]$ gas had the best average efficiency, but from Figure 9.57 it is clear that $\mathrm{He}: \mathrm{C}_{3} \mathrm{H}_{8}$ [70:30] gas has a very high constant efficiency region over most of the cell volume.

It was evident from Table 9.6 that $\mathrm{He}: \mathrm{C}_{3} \mathrm{H}_{8}[70: 30]$ gas had the best average efficiency, but from Figure 9.57 it is clear that $\mathrm{He}: \mathrm{C}_{3} \mathrm{H}_{8}[70: 30]$ gas has a very high constant efficiency region over most of the cell volume.

Next, consider a plot of resolution versus drift distance at optimum high voltage as 


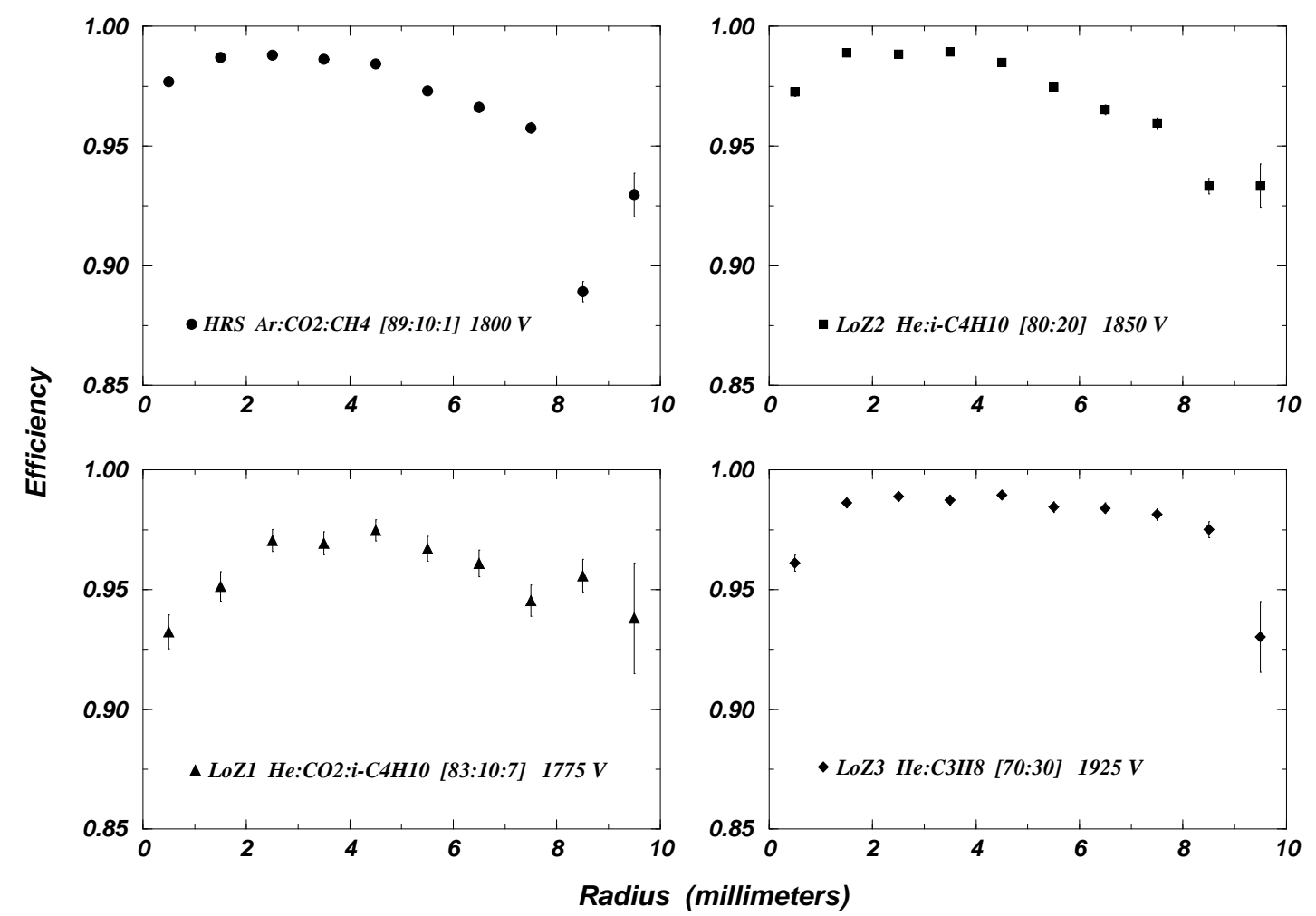

Figure 9.57: Efficiency vs. drift distance at the optimum operating high voltage for each gas. Notice that $\mathrm{He}: \mathrm{C}_{3} \mathrm{H}_{8}[70: 30]$ gas has the highest efficiency overall and a very constant efficiency region over most of the cell volume.

shown in Figure 9.58. He: $i-\mathrm{C}_{4} \mathrm{H}_{10}$ [80:20] gas gives spatial resolutions of under $100 \mu \mathrm{m}$ over $4.5 \mathrm{~mm}$ of the middle part of the cell, whereas, the $\mathrm{Ar}: \mathrm{CO}_{2}: \mathrm{CH}_{4}$ [89:10:1] and He: $\mathrm{CO}_{2}: i-\mathrm{C}_{4} \mathrm{H}_{10}$ [83:10:7] gas mixtures never fall below $100 \mu \mathrm{m}$ at any point in the cell. The absolute minimum spatial resolution operating at the optimum high voltage for each gas that was measured was $(81.3 \pm 1.8) \mu \mathrm{m}$ for $\mathrm{He}: \mathrm{i}_{4} \mathrm{C}_{4} \mathrm{H}_{10}[80: 20] ; \mathrm{He}: \mathrm{C}_{3} \mathrm{H}_{8}$ [70:30] gas was comparable with a minimum resolution of $(83.1 \pm 3.1) \mu \mathrm{m}$, but it does not quite hold the flat plateau that $\mathrm{He}: i-\mathrm{C}_{4} \mathrm{H}_{10}[80: 20]$ does. 


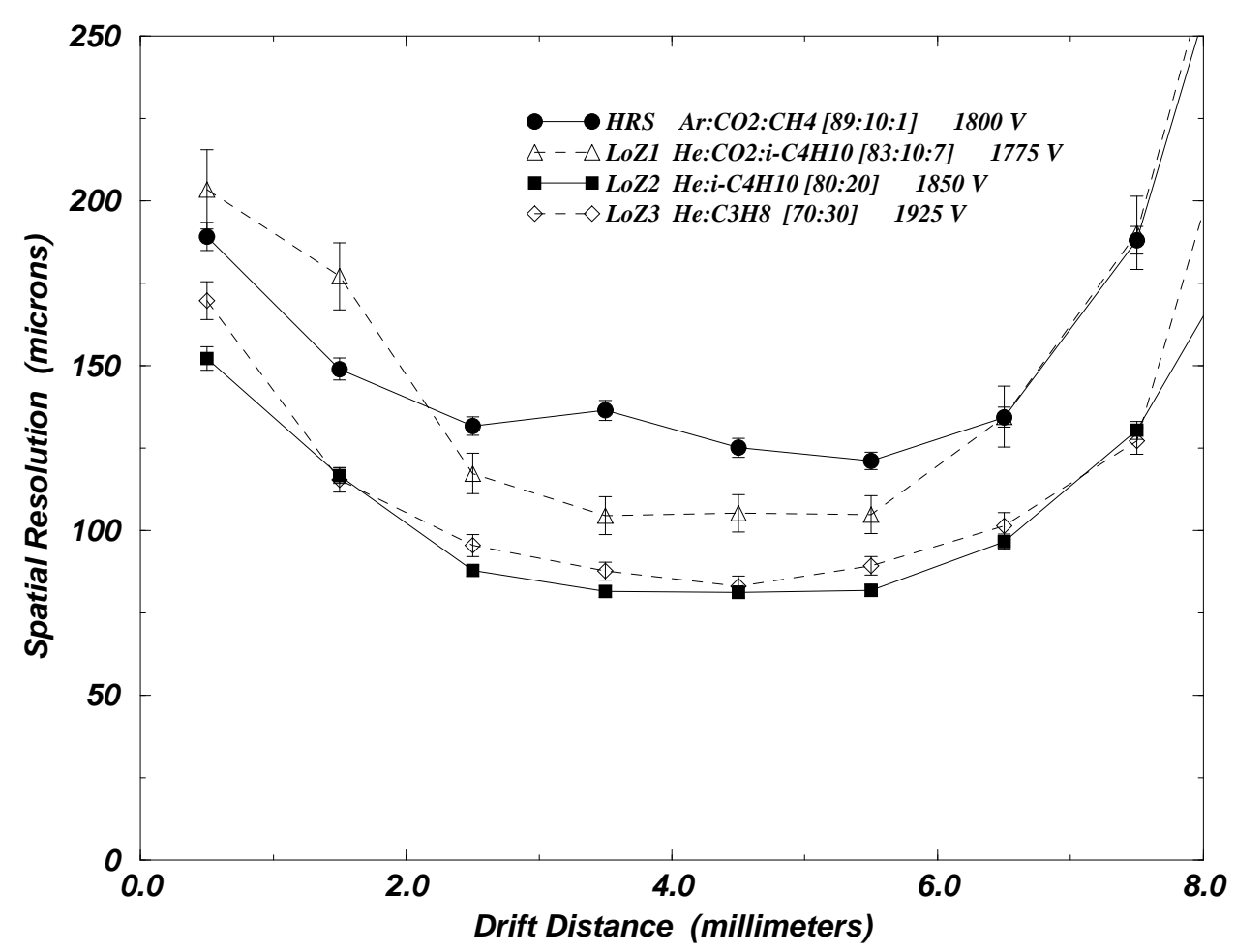

Figure 9.58: Resolution vs. drift distance at the optimum operating high voltage for each gas. Notice that the $\mathrm{He}: i-\mathrm{C}_{4} \mathrm{H}_{10}[80: 20]$ gas mixture maintains a low resolution plateau over a large fraction of the cell area.

\section{$9.2 \quad$ Future Studies}

Possible future helium-based gas mixtures that might be considered include $\mathrm{He}_{\mathrm{CF}} \mathrm{CF}_{4}$ $\mathrm{C}_{4} \mathrm{H}_{10}$ [80:10:10] gas in order to test the quenching ability of $\mathrm{CF}_{4}$ and $\mathrm{He}: \mathrm{C}_{2} \mathrm{H}_{6}$ [50:50].

Furthermore, in the spring of 1996, a second full-length prototype chamber will be built. It will be constructed using an aluminum endplate in the backward direction and a carbon-fibre endplate in the forward direction, new crimp-style feedthroughs, and gold-plated aluminum cathode wires replacing the unplated aluminum wires that were used in the first prototype. The cell shape for the new prototype will also be hexagonal, but with a slightly different ratio of anode to cathode wires. 


\subsection{Conclusion}

In conclusion, it can be stated quite clearly that helium-based gases have been shown to be a viable alternative to argon-based gases, even performing substantially better than argon-based gas mixtures according to the criteria used.

Of the four gases tested, the $\mathrm{He}: i-\mathrm{C}_{4} \mathrm{H}_{10}$ [80:20] mixture was chosen to be the best according to the resolution criterion outlined above, while $\mathrm{He}: \mathrm{C}_{3} \mathrm{H}_{8}$ [70:30] was shown to be the best gas mixture with regard to the efficiency criterion. To chose between these two in order to pick one gas as the best overall is difficult. Factors that were not addressed by this study may in the future allow for a definitive choice to be made. Such factors include the effects due to gas aging and a comparison of particle identification based on the energy loss resolution, $(d E / d x)$, for the various gases. 


\section{Bibliography}

[1] A.D. Sakharov, "Baryon asymmetry of the universe", Sov. Phys. Usp. 34 (5) (1991).

[2] Particle Data Group, L. Montanet et al., Physical Review D 50, 1173 (1994).

[3] H.R. Quinn, " $C P$ violation in $B$ physics - an overview", B Factories: The State of the Art in Accelerators, Detectors and Physics, SLAC-400 (1992).

[4] T.D. Lee and C.N. Yang, "Question of parity conservation in weak interactions", Physical Review 104, 254 (1956).

[5] C.S. Wu et al., "Experimental test of parity conservation in beta decay", Physical Review 105, 1413 (1957).

[6] J.H. Christenson, J.W. Cronin, V.L. Fitch and R. Turlay, "Evidence for the $2 \pi$ decay of the $K_{2}^{0}$ meson", Physical Review Letters, 13, 138 (1964).

[7] D. Griffiths, Introduction to Elementary Particles, Wiley \& Sons, 1987.

[8] L. Wolfenstein, "Present status of CP violation", Annual Review of Nuclear and Particle Science 36, 137 (1986).

[9] Y. Nir and H.R. Quinn, "CP violation in B physics", Annual Review of Nuclear and Particle Science 42, 211 (1992).

[10] P.R. Burchat, Physics at an Asymmetric Energy B Meson Factory, Particle Physics - The Factory Era: Lake Louise Conference proceedings, B.A. Campbell, A.N. Kamel, P. Kitching, F.C. Khanna, Eds., World Scientific, 1991.

[11] J.L. Rosner, Present and Future Aspects of CP Violation, Swieca Summer School Proceedings: Particles and Fields, World Scientific, 1995.

[12] R.L. Liboff, Introductory Quantum Mechanics, second edition, Addison-Wesley (1992).

[13] R.V. Churchill and J.W. Ward, Complex Variables and Applications, fifth edition, McGraw-Hill, 1990.

[14] The BABAR Collaboration, BABAR Technical Design Report, SLAC-R-95-457 (1995). 
[15] P. Oddone, in Proceedings of the UCLA Workshop: Linear Collider $B \bar{B}$ Factory Conceptual Design, D. Stork, Ed., World Scientific, 1987.

[16] W.R. Leo, Techniques for Nuclear and Particle Physics Experiments, SpringerVerlag, 1992.

[17] R.C. Weast and S.M. Selby, Eds., CRC Handbook of Chemistry and Physics, fortyeightth edition, The Chemical Rubber Company, 1967.

[18] P. Rice-Evans, Spark, Streamer, Proportional and Drift Chambers, The Richelieu Press, London, 1974.

[19] G. Charpak, "Drift Chambers", CERN-74-1621 (1974).

[20] S. Uno, et al., "Study of a drift chamber filled with a helium-ethane mixture", Nuclear Instruments and Methods in Physics Research A330, 55 (1993).

[21] The BABAR Collaboration, Letter of Intent for the Study of CP Violation and Heavy Flavor Physics at PEP-II, SLAC-443 (1994).

[22] P.F. Kunz, G.B. Word, The Cheetah Data Management System, SLAC-PUB 5930 (1992).

[23] P.R. Burchat et al., "Helium gas mixtures in drift chambers", Nuclear Instruments and Methods in Physics Research A316, 217 (1992).

[24] P.R. Bevington, Data Reduction and Error Analysis for the Physical Sciences, McGraw-Hill, 1969.

[25] P. Avery, Applied Fitting Theory I: General Least Squares Theory, CLEO Internal Note: CBX 91-72 (1991).

[26] P. Avery, Applied Fitting Theory IV: Formulas for Track Fitting, CLEO Internal Note: CBX 92-45 (1992).

[27] CERN, MINUIT-Function Minimization and Error Analysis, CERN Program Library Entry D506, 1992. 


\section{Appendix A}

\section{The $B A B A R$ Collaboration}

Below is a complete list of the 488 BABAR collaborators as of March 1995 when the

Technical Design Report was published. They represent 88 separate institutions from

10 countries all over the world.

LAPP Annecy, Annecy-le-Vieux, France

D. Boutigny, Y. Karyotakis, S. Lees-Rosier, P. Petitpas

INFN, Sezione di Bari and Università di Bari, Bari, Italy

C. Evangelista, A. Palano

Beijing Glass Research Institute, Beijing, China

G. Chen, Y.T. Wang, O. Wen

Institute of High Energy Physics, Beijing, China

Y.N. Guo, H.B. Lan, H.S. Mao, N.D. Qi, W.G. Yan, C.C. Zhang, W.R. Zhao,

Y.S. Zhu

University of Bristol, Bristol, UK

N. Dyce, B. Foster, R.S. Gilmore, C.J.S. Morgado

University of Bergen, Bergen, Norway

G. Eigen 
University of British Columbia, Vancouver, British Columbia, Canada

C. Goodenough, C. Hearty, J. Heise, J.A. McKenna

Brunel University, London, UK

T. Champion, A. Hasan, A.K. McKemey

Budker Institute of Nuclear Physics, Novosibirsk, Russia

A.R. Buzykaev, V.N. Golubev, V.N. Ivanchenko, S.G. Klimenko, E.A. Kravchenko, G.M. Kolachev, A.P. Onuchin, V.S. Panin, S.I. Serednyakov, A.G. Shamov, Ya.I. Skovpen, V.I. Telnov

California Institute of Technology, Pasadena, California, USA

D.G. Hitlin, J. Oyang, F.C. Porter, M. Weaver, A.J. Weinstein, R. Zhu

University of California, Davis, Davis, California, USA

F. Rouse

University of California, IIRPA, La Jolla, California, USA

A.M. Eisner, M. Sullivan, W. Vernon, Y.-X. Wang

University of California, Irvine, Irvine, California, USA

K. Gollwitzer, A. Lankford, M. Mandelkern, G. McGrath, J. Schultz,

D. Stoker, G. Zioulas

University of California, Los Angeles, Los Angeles, California, USA

K. Arisaka, C. Buchanan, J. Kubic, W. Slater

University of California, San Diego La Jolla, California, USA

V. Sharma

University of California, Santa Barbara, Santa Barbara, California, USA

D. Bauer, D. Caldwell, A. Lu, H. Nelson, J. Richman, D. Roberts, M. Witherell, 
S. Yellin

University of California, Santa Cruz, Santa Cruz, California, USA

J. DeWitt, D. Dorfan, A.A. Grillo, C. Heusch, R.P. Johnson, E. Kashigin,

S. Kashigin, W. Kroeger, W. Lockman, K. O'Shaughnessy, H. Sadrozinski, A. Seiden, E. Spencer

Carleton University and CRPP ${ }^{\dagger}$, Ottawa, Ontario, Canada

K. Edwards, D. Karlen, M. O'Neill ${ }^{\dagger}$

University of Cincinnati, Cincinnati, Ohio, USA

S. Devmal, B.T. Meadows, A.K.S. Santha, M.D. Sokoloff

University of Colorado, Boulder, Colorado, USA

A. Barker, B. Broomer, E. Erdos, W. Ford, U. Nauenberg, H. Park, P. Rankin, J. Roy, J.G. Smith

Colorado State University, Fort Collins, Colorado, USA

J. Harton, R. Malchow, M. Smy, H. Staengle, W. Toki, D. Warner, R. Wilson

Technische Universität Dresden, Institut für Kern- und Teilchenphysik, Dresden, Germany

J. Brose, G. Dahlinger, P. Eckstein, K.R. Schubert, R. Schwierz, R. Seitz, R. Waldi

Joint Institute for Nuclear Research, Dubna, Russia

A. Bannikov, S. Baranov, I. Boyko, G. Chelkov, V. Dodonov, Yu. Gornushkin,

M. Ignatenko, N. Khovansky, Z. Krumstein, V. Malyshev, M. Nikolenko, A. Nozdrin, Yu. Sedykh, A. Sissakian, Z. Silagadze, V. Tokmenin, Yu. Yatsunenko

University of Edinburgh, Edinburgh, UK

K. Peach, A. Walker 
INFN, Sezione di Ferrara, Ferrara, Italy

L. Piemontese

Laboratori Nazionali di Frascati dell' INFN, Frascati, Italy

R. Baldini, A. Calcaterra, R. De Sangro, I. Peruzzi (also Univ. Perugia), M. Piccolo, A. Zallo

INFN, Sezione di Genova and Università di Genova, Genova, Italy

A. Buzzo, R. Contri, G. Crosetti, P. Fabbricatore, S. Farinon, R. Monge, M. Olcese, R. Parodi, S. Passaggio, C. Patrignani, M.G. Pia, C. Salvo, A. Santroni

University of Iowa, Iowa City, Iowa, USA

U. Mallik, E. McCliment, M.-Z. Wang

Iowa State University, Ames, Iowa, USA

H.B. Crawley, A. Firestone, J.W. Lamsa, R. McKay, W.T. Meyer, E.I. Rosenberg

Northern Kentucky University, Highland Heights, Kentucky, USA

M. Falbo-Kenkel

University of Lancaster, Lancaster, UK

C.K. Bowdery, A.J. Finch, F. Foster

Lawrence Berkeley Laboratory, Berkeley, California, USA

G.S. Abrams, D. Brown, T. Collins, C.T. Day, S.F. Dow, F. Goozen, R. Jacobsen, R.C. Jared, J. Kadyk, L.T. Kerth, I. Kipnis, J.F. Kral, R. Lafever, R. Lee, M. Levi, L. Luo, G.R. Lynch, M. Momayezi, M. Nyman, P.J. Oddone, W.L. Pope, M. Pripstein, D.R. Quarrie, J. Rasson, N.A. Roe, M.T. Ronan, W.A. Wenzel, S. Wunduke

Lawrence Livermore National Laboratory, Livermore, California, USA

O. Alford, J. Berg, R.M. Bionta, A. Brooks, F.S. Dietrich, O.D. Fackler, 
M.N. Kreisler, M.A. Libkind, M.J. Mugge, T. O'Connor, L. Pedrotti, X. Shi, W. Stoeffl, K. van Bibber, T.J. Wenaus, D.M. Wright, C.R. Wuest, R.M. Yamamoto University of Liverpool, Liverpool, $U K$

J.R. Fry, E. Gabathuler, R. Gamet, A. Muir, P. Sanders

University of London, Imperial College of Science, Technology and

Medicine, London, UK

P. Dornan, A. Duane, L. Moneta, J. Nash, D. Price

University of London, Queen Mary \& Westfield College, London, UK

D.V. Bugg, P.F. Harrison, I. Scott, B. Zou

University of London, Royal Holloway \& Bedford New College, Egham, Surrey, UK

Y. Gao, M.G. Green, D.L. Johnson, E. Tetteh-Lartey

University of Louisville, Louisville, Kentucky, USA

C.L. Davis

McGill University, Montréal, Quebec, Canada

D. Britton, R. Fernholz, D. MacFarlane, P. Patel, C. Smith, B. Spaan, J. Trischuk University of Manchester, Manchester, UK

J. Allison, R. Barlow, G. Lafferty, K. Stephens

University of Maryland, College Park, Maryland, USA

C. Dallapiccola, M. Foucher, H. Jawahery, A. Skuja

University of Massachusetts, Amherst, Massachusetts, USA

J. Button-Shafer, J.-J. Gomez-Cadenas, S.S. Hertzbach, R.R. Kofler, M.G. Strauss

Massachusetts Institute of Technology, Cambridge, Massachusetts, USA 
R.F. Cowan, M.J. Fero, R.K. Yamamoto

INFN, Sezione di Milano and Università di Milano, Milano, Italy

M. Calvi, C. Cattadori, R. Diaferia, F. Lanni, C. Matteuzzi, F. Palombo, A. Sala, T. Tabarelli

University of Mississippi, Oxford, Mississippi, USA

M. Booke, S. Bracker, L. Cremaldi, K. Gounder, R. Kroeger, J. Reidy, D. Summers

Université de Montréal, Montréal, Quebec, Canada

G. Beaudoin, M. Beaulieu, B. Lorazo, J.P. Martin, P. Taras, V. Zacek

Mount Holyoke College, South Hadley, Massachusetts, USA

H. Nicholson, C.S. Sutton

INFN, Sezione di Napoli and Università di Napoli, Napoli, Italy

N. Cavallo, L. Lista, S. Mele, P. Parascandolo, C. Sciacca

University of Notre Dame, Notre Dame, Indiana, USA

J.M. Bishop, N.N. Biswas, N.M. Cason, J.M. LoSecco, A.H. Sanjari, W.D. Shephard

Oak Ridge National Laboratory/Y-12, Oak Ridge, Tennessee, USA

F.S. Alsmiller, R.G. Alsmiller, Jr., T.A. Gabriel, J.L. Heck

LAL Orsay, Orsay, France

D. Breton, R. Cizeron, S. Du, A.-M. Lutz, J.M. Noppe, S. Plaszczynski, M.-H.

Schune,

E. Torassa, K. Truong, G. Wormser

INFN, Sezione di Padova and Università di Padova, Padova, Italy

F. Dal Corso, M. Morandin, M. Posocco, R. Stroili, C. Voci

Ecole Polytechnique Palaiseau, LPNHE, Palaiseau, France 
L. Behr, G. Bonneaud, P. Matricon, G. Vasileiadis, M. Verderi

LPNHE des Universités Paris 6 et Paris 7, Paris, France

M. Benayoun, H. Briand, J. Chauveau, P. David, C. De La Vaissiere, L. Del Buono,

J.F. Genat, O. Hamon, P. Leruste, J. Lory, J.-L. Narjoux, B. Zhang

INFN, Sezione di Milano and Università di Pavia, Pavia, Italy

P.F. Manfredi, V. Re, V. Speziali, F. Svelto

University of Pennsylvania, Philadelphia, Pennsylvania, USA

L. Gladney

INFN, Sezione di Pisa, Università di Pisa ${ }^{\dagger}$ and Scuola Normale Superiore ${ }^{\ddagger}$, Pisa, Italy

G. Batignani ${ }^{\dagger}$, S. Bettarini, F. Bosi, U. Bottigli ${ }^{\dagger}$, M. Carpinelli, F. Costantini ${ }^{\dagger}$,

F. Forti, D. Gambino, M. Giorgi ${ }^{\dagger}$, A. Lusiani ${ }^{\ddagger}$, P.S. Marrocchesi, M. Morganti ${ }^{\dagger}$,

G. Rizzo, G. Triggiani ${ }^{\dagger}$, J. Walsh

Prairie View A\&M University, Prairie View, Texas, USA

M. Gui, D.J. Judd, K. Paick, D.E. Wagoner

Princeton University, Princeton, New Jersey, USA

C. Bula, C. Lu, K.T. McDonald

INFN, Instituto Superiore di Sanità, Roma, Italy

C. Bosio

INFN, Sezione di Roma and Università "La Sapienza," Roma, Italy

F. Ferroni, E. Lamanna, M.A. Mazzoni, S. Morganti, G. Piredda, R. Santacesaria

Rutgers University, Rutgers, New Jersey, USA

P. Jacques, M. Kalelkar, R. Plano, P. Stamer 
Rutherford Appleton Laboratory, Chilton, Didcot, UK

P.D. Dauncey, J. Dowdell, B. Franek, N.I. Geddes, G.P. Gopal, R. Halsall, J.A. Lidbury, V.J. Perera

CEA, DAPNIA, CE-Saclay, ${ }^{1}$ Gif-sur-Yvette, France

R. Aleksan, P. Besson, T. Bolognese, P. Bourgeois, A. de Lesquen, A. Gaidot,

L. Gosset,

G. Hamel de Monchenault, P. Jarry, G. London, M. Turluer, G. Vasseur, C. Yeche, M. Zito

Shanghai Institute of Ceramics (SICCAS), Shanghai, China

J.R. Jing, P.J. Li, D.S. Yan, Z.W. Yin

University of South Carolina, Columbia, South Carolina, USA

M.V. Purohit, J. Wilson

Stanford Linear Accelerator Center, Stanford, California, USA

D. Aston, R. Becker-Szendy, R. Bell, E. Bloom, C. Boeheim, A. Boyarski,

R.F. Boyce, D. Briggs, F. Bulos, W. Burgess, R.L.A. Cottrell, D.H. Coward,

D.P. Coupal, W. Craddock, H. DeStaebler, J.M. Dorfan, W. Dunwoodie, T. Fieguth,

D. Freytag, R. Gearhart, T. Glanzman, G. Godfrey, G. Haller, J. Hewett, T. Himel,

J. Hoeflich, W. Innes, C.P. Jessop, W.B. Johnson, H. Kawahara, L. Keller, ${ }^{2}$

M.E. King, J. Krebs, P. Kunz, W. Langeveld, E. Lee, D.W.G.S. Leith, V.G. Lüth,

H. Lynch, H. Marsiske, T. Mattison, R. Melen, K. Moffeit, L. Moss, D. Muller,

M. Perl, G. Oxoby, M. Pertsova, H. Quinn, B.N. Ratcliff, S.F. Schaffner,

R.H. Schindler, S. Shapiro, C. Simopolous, A.E. Snyder, E.J. Soderstrom, J. Vav'ra,

S. Wagner, D. Walz, R. Wang, J.L. White, W. Wisniewski, N. Yu

\footnotetext{
${ }^{1}$ Subject to approval of funding agency.

${ }^{2}$ Retired
} 
Stanford University, Stanford, California, USA

P. Burchat, R. Zaliznyak

Academia Sinica, Taipei, Taiwan

H.-Y. Chau, M.-L. Chu, S.-C. Lee

University of Texas at Dallas, Richardson, Texas, USA

J.M. Izen, X. Lou

INFN, Sezione di Torino and Università di Torino, Torino, Italy

F. Bianchi, D. Gamba, G. Giraudo, A. Romero

INFN, Sezione di Trieste and Università di Trieste, Trieste, Italy

L. Bosisio, R. Della Marina, G. Della Ricca, B. Gobbo, L. Lanceri, P. Poropat

TRIUMF, Vancouver, British Columbia, Canada

R. Henderson, A. Trudel

Tsinghua University, Beijing, China

Y.P. Kuang, R.C. Shang, B.B. Shao, J.J. Wang

Vanderbilt University, Nashville, Tennessee, USA

R.S. Panvini, T.W. Reeves, P.D. Sheldon, M.S. Webster

University of Victoria, Victoria, British Columbia, Canada

M. McDougald, D. Pitman

University of Wisconsin, Madison, Wisconsin, USA

H.R. Band, J.R. Johnson, R. Prepost, G.H. Zapalac

York University, Toronto, Ontario, Canada

W. Frisken 